\title{
On Single Valued Neutrosophic Regularity Spaces
}

\author{
Yaser Saber $^{1,2}$, Fahad Alsharari ${ }^{1,6, *}$, Florentin Smarandache ${ }^{3}$ and Mohammed Abdel-Sattar ${ }^{4,5}$ \\ ${ }^{1}$ Department of Mathematics, College of Science and Human Studies, Hotat Sudair, Majmaah University, Majmaah, 11952, \\ Saudi Arabia \\ ${ }^{2}$ Department of Mathematics, Faculty of Science, Al-Azhar University, Assiut, 71524, Egypt \\ ${ }^{3}$ Department of Mathematics, University of New Mexico, Gallup, NM 87301, USA \\ ${ }^{4}$ Department of Mathematics, College of Science and Arts King Khaled University, Mhayal Asier, 61913, Saudi Arabia \\ ${ }^{5}$ Department of Mathematics and Computer Science, Faculty of Science, Beni-Suef University, Beni-Suef, 62511, Egypt \\ ${ }^{6}$ Department of Mathematics, College of Science and Arts, Jouf University, Gurayat, 77455, Saudi Arabia \\ ${ }^{*}$ Corresponding Author: Fahad Alsharari. Email: f.alsharari@mu.edu.sa
}

Received: 06 June 2021 Accepted: 03 August 2021

\begin{abstract}
This article aims to present new terms of single-valued neutrosophic notions in the Šostak sense, known as singlevalued neutrosophic regularity spaces. Concepts such as $r$-single-valued neutrosophic semi $£$-open, $r$-single-valued neutrosophic pre-f-open, $r$-single valued neutrosophic regular- $£$-open and $r$-single valued neutrosophic $\alpha £$-open are defined and their properties are studied as well as the relationship between them. Moreover, we introduce the concept of $r$-single valued neutrosophic $\theta £$-cluster point and $r$-single-valued neutrosophic $\gamma £$-cluster point, $r-\theta £$ closed, and $\theta £$-closure operators and study some of their properties. Also, we present and investigate the notions of $r$-single-valued neutrosophic $\theta £$-connectedness and $r$-single valued neutrosophic $\delta £$-connectedness and investigate relationship with single-valued neutrosophic almost $£$-regular. We compare all these forms of connectedness and investigate their properties in single-valued neutrosophic semiregular and single-valued neutrosophic almost regular in neutrosophic ideal topological spaces in Šostak sense. The usefulness of these concepts are incorporated to multiple attribute groups of comparison within the connectedness and separateness of $\theta £$ and $\delta £$.
\end{abstract}

\section{KEYWORDS}

Single valued neutrosophic $\theta £$-closed; single valued neutrosophic $\theta £$-separated; single valued neutrosophic $\delta £$-separated; single-valued neutrosophic $\delta £$-connected; single valued neutrosophic $\delta £$-connected; single valued neutrosophic almost $£$-egular

\section{Introduction}

A neutrosophic set can be practical in addressing problems with indeterminate, imperfect, and inconsistent materials. The concept of neutrosophic set theory was introduced by Smarandache [1] as a new mathematical method that corresponds to the indeterminacy degree (uncertainty, etc.). Bakbak et al. [2] and Mishra et al. [3] applied the soft set theory successfully applied in several

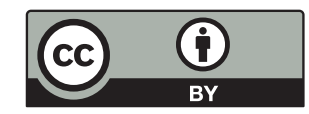

This work is licensed under a Creative Commons Attribution 4.0 International License, which permits unrestricted use, distribution, and reproduction in any medium, provided the original work is properly cited. 
areas, such as the smoothness of functions, as well as architecture-based, neuro-linguistic programming. Wang et al. [4] proposed single-valued neutrosophic sets (SVNSs). Meanwhile, Kim et al. $[5,6]$ inspected the single valued neutrosophic relations (SVNRs) and symmetric closure of SVNR, respectively. Recently, Saber et al. [7-9] introduced the concepts of single-valued neutrosophic ideal open local function and single-valued neutrosophic topological space. Many of their applications appear in the studies of Das et al. [10]. Alsharari et al. [11-13]. Riaz et al. [14]. Salama et al. [15-17]. Hur et al. [18,19]. Yang et al. [20]. El-Gayyar [21], AL-Nafee et al. [22]. Muhiuddin et al. [23,24] and Mukherjee et al. [25].

First, we define single-valued neutrosophic $\theta £$-closed and single-valued neutrosophic $\delta £$-closed sets as well as some of their core properties. We also present and explore the properties and characterizations of single valued neutrosophic operators namely $\theta £$-closure $\left(\mathrm{CI}_{\tilde{\tau} \tilde{\varrho} \tilde{\sigma} \tilde{\xi}}^{\theta £}\right)$ and $\delta £$ closure $\left(\mathrm{CI}_{\tilde{\tau} \tilde{\varrho} \tilde{\sigma} \tilde{\sigma}}^{\delta £}\right)$ in the single valued neutrosophic ideal topological space $\left(\tilde{\mathcal{F}}, \tau^{\tilde{\varrho} \tilde{\sigma} \tilde{\zeta}}, £^{\tilde{\varrho} \tilde{\sigma} \tilde{\zeta}}\right)$. We then define the concept of single valued neutrosophic regularity spaces. Next, we study singlevalued neutrosophic $\theta £$-separated and single-valued neutrosophic $\delta £$-separated with giving some definitions and theorems. Furthermore, we also introduce single-valued neutrosophic $\theta \mathfrak{f}$-connected and single valued neutrosophic $\delta £$-connected relying on the single valued neutrosophic $\theta £$-closure and $\delta £$-closure operators.

We define a fixed universe $\tilde{\mathcal{F}}$ to be a finite set of objects and $\zeta$ a closed unit interval [0, 1]. Additionally, we denote $\zeta^{\mathcal{F}}$ as the set of all single-valued neutrosophic subsets of $\tilde{\mathcal{F}}$.

\section{Preliminaries}

This section provides a complete survey, some previous studies, and concepts associated with this study.

Definition 1. [1] Let $\tilde{\mathcal{F}}$ be a non-empty set. A neutrosophic set (briefly, $\mathcal{N} \mathcal{S}$ ) in $\tilde{\mathcal{F}}$ is an object having the form $\alpha_{n}=\left\{\left\langle v, \tilde{\varrho}_{\alpha_{n}}(v), \tilde{\sigma}_{\sigma_{n}}(\omega), \tilde{\zeta}_{\alpha_{n}}(v)\right\rangle: v \in \tilde{\mathcal{F}}\right\}$ where

$\tilde{\varrho}: \tilde{\mathcal{F}} \rightarrow\rfloor^{-} \mathbf{0}, \mathbf{1}^{+}\lfloor, \tilde{\boldsymbol{\sigma}}: \tilde{\mathcal{F}} \rightarrow\rfloor^{-} \mathbf{0}, \mathbf{1}^{+}\lfloor, \tilde{\boldsymbol{\zeta}}: \tilde{\mathcal{F}} \rightarrow\rfloor^{-} \mathbf{0}, \mathbf{1}^{+}\left\lfloor\right.$and ${ }^{-} \mathbf{0} \leq \tilde{\varrho}_{\alpha_{n}}(v)+\tilde{\boldsymbol{\sigma}}_{\alpha_{n}}(\boldsymbol{v})+\tilde{\boldsymbol{S}}_{\boldsymbol{\alpha}_{n}}(\boldsymbol{v}) \leq \mathbf{3}^{+}$

Represent the degree of membership $\left(\tilde{\varrho}_{\alpha_{n}}\right)$, the degree of indeterminacy $\left(\tilde{\sigma}_{\alpha_{n}}\right)$, and the degree of non-membership $\left(\tilde{\zeta}_{\alpha_{n}}\right)$ respectively of any $v \in \tilde{\mathcal{F}}$ to the set $\alpha_{n}$.

Definition 2. [4] Suppose that $\widetilde{\mathcal{F}}$ is a universal set a space of points (objects), with a generic element in $\tilde{\mathcal{F}}$ denoted by $v$. Then $\alpha_{n}$ is called a single valued neutrosophic set (briefly, $\mathcal{S} \mathcal{V N S}$ ) in $\widetilde{\mathcal{F}}$, if $\alpha_{n}$ has the form $\alpha_{n}=\left\{\left\langle v, \tilde{\varrho}_{\alpha_{n}}(v), \tilde{\sigma}_{\alpha_{n}}(v), \tilde{\zeta}_{\alpha_{n}}(v)\right\rangle: v \in \tilde{\mathcal{F}}\right\}$. Now, $\tilde{\varrho}_{\alpha_{n}}, \tilde{\sigma}_{\sigma_{n}}, \tilde{\zeta}_{\alpha_{n}}$ indicate the degree of non-membership, the degree of indeterminacy, and the degree of membership, respectively of any $v \in \tilde{\mathcal{F}}$ to the set $\alpha_{n}$.

Definition 3. [4] Let $\alpha_{n}=\left\{\left\langle v, \tilde{\varrho}_{\alpha_{n}}(v), \tilde{\sigma}_{\sigma_{n}}(v), \tilde{\zeta}_{\alpha_{n}}(v)\right\rangle: v \in \tilde{\mathcal{F}}\right\}$ be an SVNS on $\tilde{\mathcal{F}}$. The complement of the set $\alpha_{n}$ (briefly, $\left.\alpha_{n}^{c}\right)$ defined as follows: $\tilde{\varrho}_{\alpha_{n}^{c}}(v)=\tilde{\zeta}_{\alpha_{n}}(v), \tilde{\sigma}_{\alpha_{n}}(v)=\left[\tilde{\sigma}_{\alpha_{n}}\right]^{c}(v), \tilde{\zeta}_{\alpha_{n}^{c}}(v)=$ $\tilde{\varrho}_{\alpha_{n}}(v)$.

Definition 4. [26] Let $\tilde{\mathcal{F}}$ be a non-empty set and $\alpha_{n}, \varepsilon_{n} \in \zeta^{\tilde{\mathcal{F}}}$ be in the form: $\alpha_{n}=$ $\left\{\left\langle v, \tilde{\varrho}_{\alpha_{n}}(v), \tilde{\sigma}_{\alpha_{n}}(v), \tilde{\zeta}_{\alpha_{n}}(v)\right\rangle: v \in \tilde{\mathcal{F}}\right\}$ and $\varepsilon_{n}=\left\{\left\langle v, \tilde{\varrho}_{\varepsilon_{n}}(v), \tilde{\sigma}_{\varepsilon_{n}}(v), \tilde{\zeta}_{\varepsilon_{n}}(v)\right\rangle: v \in \tilde{\mathcal{F}}\right\}$ on $\tilde{\mathcal{F}}$ then,

(a) $\alpha_{n} \subseteq \varepsilon_{n}$ for every $v \in \tilde{\mathcal{F}} ; \tilde{\varrho}_{\alpha_{n}}(v) \leq \tilde{\varrho}_{\varepsilon_{n}}(v), \tilde{\sigma}_{\alpha_{n}}(v) \geq \tilde{\sigma}_{\varepsilon_{n}}(v), \tilde{\zeta}_{\alpha_{n}}(v) \geq \tilde{\zeta}_{\varepsilon_{n}}(v)$.

(b) $\alpha_{n}=\varepsilon_{n}$ iff $\sigma_{n} \subseteq \varepsilon_{n}$ and $\sigma_{n} \supseteq \varepsilon_{n}$.

(c) $\tilde{0}=\langle 0,1,1\rangle$ and $\tilde{1}=\langle 1,0,0\rangle$. 
Definition 5. [20] Let $\alpha_{n}, \varepsilon_{n} \in \zeta^{\tilde{\mathcal{F}}}$. Then,

(a) $\alpha_{n} \cap \varepsilon_{n}$ is an SVNS, if for every $v \in \tilde{\mathcal{F}}$,

$\boldsymbol{\alpha}_{n} \cap \boldsymbol{\varepsilon}_{\boldsymbol{n}}=\left\langle\left(\tilde{\varrho}_{\alpha_{n}} \cap \tilde{\varrho}_{\varepsilon_{n}}\right)(v),\left(\tilde{\sigma}_{\alpha_{n}} \cup \tilde{\sigma}_{\varepsilon_{n}}\right)(v),\left(\tilde{\boldsymbol{\zeta}}_{\alpha_{n}} \cup \tilde{\boldsymbol{\zeta}}_{\varepsilon_{n}}\right)(\boldsymbol{v})\right\rangle$

where, $\left(\tilde{\varrho}_{\alpha_{n}} \cap \tilde{\varrho}_{\varepsilon_{n}}\right)(v)=\tilde{\varrho}_{\alpha_{n}}(v) \cap \tilde{\varrho}_{\varepsilon_{n}}(v)$ and $\left(\tilde{\zeta}_{\alpha_{n}} \cup \tilde{\zeta}_{\varepsilon_{n}}\right)(v)=\tilde{\zeta}_{\alpha_{n}}(v) \cup \tilde{\zeta}_{\varepsilon_{n}}(v)$, for all $v \in \tilde{\mathcal{F}}$,

(b) $\alpha_{n} \cup \varepsilon_{n}$ is an SVNS, if for every $v \in \tilde{\mathcal{F}}$,

$\alpha_{n} \cup \varepsilon_{n}=\left\langle\left(\tilde{\varrho}_{\alpha_{n}} \cup \tilde{\varrho}_{\varepsilon_{n}}\right)(v),\left(\tilde{\sigma}_{\alpha_{n}} \cap \tilde{\sigma}_{\varepsilon_{n}}\right)(v),\left(\tilde{\zeta}_{\alpha_{n}} \cap \tilde{\boldsymbol{\zeta}}_{\varepsilon_{n}}\right)(v)\right\rangle$.

Definition 6. [15] For an any arbitrary family $\left\{\alpha_{n}\right\}_{i \in j} \in \zeta^{\tilde{\mathcal{F}}}$ of SVNS the union and intersection are given by

(a) $\bigcap_{i \in j}\left[\alpha_{n}\right]_{i}=\left\langle\cap_{i \in j} \tilde{\varrho}_{\left[\alpha_{n}\right]_{i}}(v), \cup_{i \in j} \tilde{\sigma}_{\left[\alpha_{n}\right]_{i}}(v), \cup_{i \in j} \tilde{S}_{\left[\alpha_{n}\right]_{i}}(v)\right\rangle$,

(b) $\bigcup_{i \in j}\left[\alpha_{n}\right]_{i}=\left\langle\bigcup_{i \in j} \tilde{\varrho}_{\left[\alpha_{n}\right]_{i}}(v), \cap_{i \in j} \tilde{\sigma}_{\left[\alpha_{n}\right]_{i}}(v), \cap_{i \in j} \tilde{\zeta}\left[\alpha_{n}\right]_{i}(v)\right\rangle$.

Definition 7. [21] A single-valued neutrosophic topological spaces is an ordered $\left(\tilde{\mathcal{F}}, \tilde{\tau}^{\tilde{\varrho}}, \tilde{\tau}^{\tilde{\sigma}}, \tilde{\tau}^{\tilde{\varsigma}}\right)$ where $\tilde{\tau}^{\tilde{\varrho}}, \tilde{\tau}^{\tilde{\sigma}}, \tilde{\tau} \tilde{\zeta}: \zeta^{\tilde{\mathcal{F}}} \rightarrow \zeta$ is a mapping satisfying the following axioms:

(SVNT1) $\tilde{\tau} \tilde{\varrho}(\tilde{0})=\tilde{\tau} \tilde{\varrho}(\tilde{1})=\tilde{\tau}^{\tilde{\sigma}}(\tilde{0})=\tilde{\tau} \tilde{\sigma}(\tilde{1})=0$ and $\tilde{\tau} \tilde{\varsigma}(\tilde{0})=\tilde{\tau} \tilde{\varsigma}(\tilde{1})=1$.

(SVNT2) $\tilde{\tau}^{\tilde{\varrho}}\left(\alpha_{n} \cap \varepsilon_{n}\right) \geq \tilde{\tau}^{\tilde{\varrho}}\left(\alpha_{n}\right) \cap \tilde{\tau}^{\tilde{\varrho}}\left(\varepsilon_{n}\right), \tilde{\tau}^{\tilde{\sigma}}\left(\alpha_{n} \cap \varepsilon_{n}\right) \leq \tau^{\tilde{\sigma}}\left(\alpha_{n}\right) \cup \tilde{\tau}^{\tilde{\sigma}}\left(\varepsilon_{n}\right), \tilde{\tau}^{\tilde{\zeta}}\left(\alpha_{n} \cap \varepsilon_{n}\right) \leq \tilde{\tau}^{\tilde{S}}\left(\alpha_{n}\right) \cup$ $\tilde{\tau} \tilde{\zeta}\left(\varepsilon_{n}\right)$ for every, $\alpha_{n}, \varepsilon_{n} \in \zeta^{\tilde{\mathcal{F}}}$

(SVNT3) $\tilde{\tau}^{\tilde{\varrho}}\left(\cup_{j \in \Gamma}\left[\alpha_{n}\right]_{j}\right) \geq \cap_{j \in \Gamma} \tilde{\tau} \tilde{\varrho}\left(\left[\alpha_{n}\right]_{j}\right), \tilde{\tau}^{\tilde{\sigma}}\left(\cup_{i \in \Gamma}\left[\alpha_{n}\right]_{j}\right) \leq \cup_{j \in \Gamma} \tilde{\tau}^{\tilde{\sigma}}\left(\left[\alpha_{n}\right]_{j}\right), \tilde{\tau} \tilde{s}\left(\cup_{j \in \Gamma}\left[\alpha_{n}\right]_{j}\right) \leq \cup_{j \in \Gamma} \tilde{\tau} \tilde{\tilde{s}}\left(\left[\alpha_{n}\right]_{j}\right)$, for every $\left[\alpha_{n}\right]_{j} \in \zeta^{\tilde{\mathcal{F}}}$.

The quadruple $\left(\tilde{\mathcal{F}}, \tilde{\tau}^{\tilde{\varrho}}, \tilde{\tau}^{\tilde{\sigma}}, \tilde{\tau}^{\tilde{\zeta}}\right)$ is called a single-valued neutrosophic topological spaces (briefly, SVNT, for short). Occasionally write $\tau^{\tilde{\varrho} \tilde{\sigma} \tilde{\zeta}}$ for $\left(\tilde{\tau} \tilde{\varrho}, \tilde{\tau}^{\tilde{\sigma}}, \tilde{\tau}^{\tilde{\zeta}}\right)$ and it will cause no ambiguity.

Definition 8. [7] Let $(\tilde{\mathcal{F}}, \tau \tilde{\varrho} \tilde{\sigma} \tilde{\zeta})$ be an SVNTS. Then, for every $\alpha_{n} \in \zeta^{\tilde{\mathcal{F}}}$ and $r \in \zeta_{0}$. Then the single valued neutrosophic closure and single valued neutrosophic interior of $\alpha_{n}$ are define by:

$$
\begin{aligned}
& \mathbf{C}_{\tau} \tilde{\tilde{\varrho} \tilde{\sigma} \tilde{\zeta}}\left(\alpha_{n}, r\right)=\bigcap\left\{\varepsilon_{n} \in \zeta^{\tilde{\mathcal{F}}}: \alpha_{n} \leq \varepsilon_{n}, \tau^{\tilde{\varrho}}\left(\left[\varepsilon_{n}\right]^{c}\right) \geq r, \tau^{\tilde{\sigma}}\left(\left[\varepsilon_{n}\right]^{c}\right) \leq 1-r, \tau^{\tilde{\zeta}}\left(\left[\varepsilon_{n}\right]^{c}\right) \leq 1-r\right\} \\
& \operatorname{int}_{\tau} \tilde{\tilde{\sigma} \tilde{\sigma} \tilde{s}}\left(\alpha_{n}, r\right)=\bigcup\left\{\varepsilon_{n} \in \zeta^{\tilde{\mathcal{F}}}: \alpha_{n} \geq \varepsilon_{n}, \tau^{\tilde{\varrho}}\left(\varepsilon_{n}\right) \geq r, \tau^{\tilde{\sigma}}\left(\varepsilon_{n}\right) \leq 1-r, \tau^{\tilde{S}}\left(\varepsilon_{n}\right) \leq 1-r\right\}
\end{aligned}
$$

Definition 9. [7] Let $(\tilde{\mathcal{F}})$ be a nonempty set and $v \in \tilde{\mathcal{F}}$, let $s \in(0,1], t \in[0,1)$ and $k \in[0,1)$, then the single-valued neutrosophic point $x_{s, t, k}$ in $\tilde{\mathcal{F}}$ given by $x_{s, t, k}(v)= \begin{cases}(s, t, k), & \text { if } x=v \\ (0,1,1), & \text { otherwise. }\end{cases}$

We define that, $x_{s, t, p} \in \alpha_{n}$ iff $s<\tilde{\varrho}_{\alpha_{n}}(v), t \geq \tilde{\sigma}_{\alpha_{n}}(v)$ and $k \geq \tilde{\tilde{S}}_{\alpha_{n}}(v)$. We indicate the set of all single-valued neutrosophic points in $\tilde{\mathcal{F}}$ as $\mathrm{P}_{x_{s, t, k}}(\tilde{\mathcal{F}})$. A single-valued neutrosophic set $\alpha_{n}$ is said to be quasi-coincident with another single-valued neutrosophic set $\varepsilon_{n}$, denoted by $\alpha_{n} q \varepsilon_{n}$, if there exists an element $v \in \tilde{\mathcal{F}}$ such that $\tilde{\varrho}_{\alpha_{n}}(v)+\tilde{\varrho}_{\varepsilon_{n}}(v)>1, \tilde{\sigma}_{\alpha_{n}}(v)+\tilde{\sigma}_{\varepsilon_{n}}(v) \leq 1, \tilde{\zeta}_{\alpha_{n}}(v)+\tilde{\zeta}_{\varepsilon_{n}}(v) \leq 1$. 
Definition 10. [7] A mapping $£_{\tilde{\mathscr{\rho}}}^{\tilde{\mathcal{E}}}, £^{\tilde{\sigma}}, £^{\tilde{\zeta}}: \zeta^{\tilde{\mathcal{F}}} \rightarrow \zeta$ is called single-valued neutrosophic ideal (SVNI) on $\tilde{\mathcal{F}}$ if, it satisfies the following conditions:

$\left(£_{1}\right) £^{\tilde{\varrho}}(\tilde{0})=1$ and $£^{\tilde{\sigma}}(\tilde{0})=£^{\tilde{S}}(\tilde{0})=0$.

(£2) If $\sigma_{n} \leq \gamma_{n}$, then $£^{\tilde{\varrho}}\left(\varepsilon_{n}\right) \leq £^{\tilde{\varrho}}\left(\alpha_{n}\right), £^{\tilde{\sigma}}\left(\varepsilon_{n}\right) \geq £^{\tilde{\sigma}}\left(\alpha_{n}\right)$ and $£^{\tilde{S}}\left(\varepsilon_{n}\right) \geq £^{\tilde{S}}\left(\alpha_{n}\right)$, for $\varepsilon_{n}, \alpha_{n} \in \zeta^{\tilde{\mathcal{F}}}$.

$\left(£_{3}\right) £^{\tilde{\varrho}}\left(\alpha_{n} \cup \varepsilon_{n}\right) \geq £^{\tilde{\varrho}}\left(\alpha_{n}\right) \cap £^{\tilde{\varrho}}\left(\varepsilon_{n}\right), £^{\tilde{\sigma}}\left(\alpha_{n} \cup \varepsilon_{n}\right) \leq £^{\tilde{\sigma}}\left(\alpha_{n}\right) \cup £^{\tilde{\sigma}}\left(\varepsilon_{n}\right)$ and $£^{\tilde{S}}\left(\alpha_{n} \cup \varepsilon_{n}\right) \leq £^{\tilde{S}}\left(\alpha_{n}\right) \cup £^{\tilde{S}}\left(\varepsilon_{n}\right)$, for $\alpha_{n}, \varepsilon_{n} \in \zeta^{\tilde{\mathcal{F}}}$.

The tribal $(\tilde{\mathcal{F}}, \tau \tilde{\varrho} \tilde{\sigma} \tilde{\zeta}, £ \tilde{\varrho} \tilde{\sigma} \tilde{\varsigma})$ is called a single valued neutrosophic ideal topological space in Šostak sense (briefly, SVNITS).

Definition 11. [7] Let $\left(\tilde{\mathcal{F}}, \tau^{\tilde{\varrho} \tilde{\sigma} \tilde{\zeta}}, £^{\tilde{\varrho} \tilde{\sigma} \tilde{\varsigma}}\right)$ be an SVNITS for each $\alpha_{n} \in \zeta^{\tilde{\mathcal{F}}}$. Then, the single valued neutrosophic ideal open local function $\left[\alpha_{n}\right]_{r}^{\odot}\left(\tau^{\tilde{\varrho} \tilde{\sigma} \tilde{\zeta}}, £^{\tilde{\varrho} \tilde{\sigma} \tilde{\zeta}}\right)$ of $\alpha_{n}$ is the union of all single-valued neutrosophic points $x_{s, t, k}$ such that if $\varepsilon_{n} \in Q_{\tau} \tilde{\varrho} \tilde{\sigma} \tilde{s}\left(x_{s, t, k}, r\right)$ and $£^{\tilde{\varrho}}\left(\omega_{n}\right) \geq r, £^{\tilde{\sigma}}\left(\omega_{n}\right) \leq 1-r, £^{\tilde{S}}\left(\omega_{n}\right) \leq$ $1-r$, then there is at least one $v \in \tilde{\mathcal{F}}$ for which

$\tilde{\varrho}_{\alpha_{n}}(v)+\tilde{\varrho}_{\varepsilon_{n}}(v)-1>\tilde{\varrho}_{\omega_{n}}(v), \quad \tilde{\sigma}_{\alpha_{n}}(v)+\tilde{\sigma}_{\varepsilon_{n}}(v)-1 \leq \tilde{\sigma}_{\omega_{n}}(v), \quad \tilde{\zeta}_{\alpha_{n}}(v)+\tilde{\zeta}_{\varepsilon_{n}}(v)-1 \leq \tilde{\boldsymbol{S}}_{\omega_{n}}(v)$

Occasionally, we will write $\left[\alpha_{n}\right]_{r}^{\odot}$ for $\left[\alpha_{n}\right]_{r}^{\odot}\left(\tau^{\tilde{\varrho} \tilde{\sigma} \tilde{\zeta}}, £^{\tilde{\varrho} \tilde{\sigma} \tilde{\zeta}}\right)$ herein to avoid ambiguity.

Remark 1. [7] Let $(\tilde{\mathcal{F}}, \tau \tilde{\varrho} \tilde{\sigma} \tilde{\zeta}, £ \tilde{\varrho} \tilde{\sigma} \tilde{\zeta})$ be an SVNITS and $\alpha_{n} \in \zeta^{\tilde{\mathcal{F}}}$. Hence, we can write

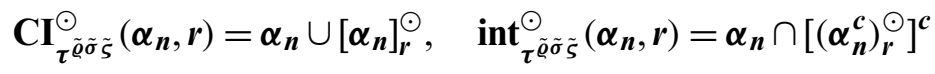

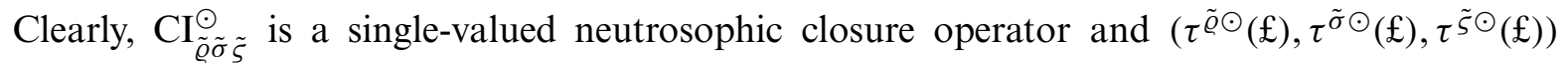
is the single-valued neutrosophic topology generated by $\mathrm{CI}_{\tau \tilde{\rho} \tilde{\sigma} \tilde{\zeta}}^{\odot}$, i.e., $\tau^{\odot}(\mathcal{J})\left(\alpha_{n}\right)=\bigcup\left\{r \mid \mathrm{CI}_{\tilde{\tau} \tilde{\rho} \tilde{\sigma} \tilde{\zeta}}^{\odot}\right.$ $\left.\left(\alpha_{n}^{c}, r\right)=\alpha_{n}^{c}\right\}$.

Theorem 1. [7] Let $\left\{\left[\alpha_{n}\right]_{i}\right\}_{i \in J} \subset \zeta^{\tilde{\mathcal{F}}}$ be a family of single-valued neutrosophic sets on $\tilde{\mathcal{F}}$ and $\left(\tilde{\mathcal{F}}, \tilde{\tau} \tilde{\varrho} \tilde{\sigma} \tilde{s}, £^{\tilde{\varrho} \tilde{\sigma} \tilde{s}}\right)$ be a SVNITS. Then,

(a) $\left(\cup\left(\left[\alpha_{n}\right]_{i}\right)_{r}^{\odot}: i \in J\right) \leq\left(\cup\left[\alpha_{n}\right]_{i}: i \in j\right)_{r}^{\odot}$,

(b) $\left(\cap\left(\left[\alpha_{n}\right]_{i}\right): i \in j\right)_{r}^{\odot} \geq\left(\cap\left(\left[\alpha_{n}\right]_{i}\right)_{r}^{\odot}: i \in J\right)$.

Theorem 2. [7] Let $\left(\tilde{\mathcal{F}}, \tau^{\tilde{\varrho} \tilde{\sigma} \tilde{\zeta}}, £^{\tilde{\varrho} \tilde{\sigma} \tilde{\zeta}}\right)$ be an SVNITS and $r \in \zeta, \alpha_{n}, \varepsilon_{n} \in \zeta^{\tilde{\mathcal{F}}}$. Then,

(a) $\operatorname{int}_{\tilde{\tau} \tilde{\rho} \tilde{\sigma} \tilde{\xi}}^{\odot}\left(\alpha_{n} \vee \varepsilon_{n}, r\right) \leq \operatorname{int}_{\tilde{\tau} \tilde{\rho} \tilde{\sigma} \tilde{\xi}}^{\odot}\left(\alpha_{n}, r\right) \vee \operatorname{int}_{\tilde{\tau} \tilde{\varrho} \tilde{\sigma} \tilde{\xi}}^{\odot}\left(\varepsilon_{n}, r\right)$

(b) $\operatorname{int}_{\tilde{\tau} \tilde{\rho} \tilde{\sigma} \tilde{s}}\left(\alpha_{n}, r\right) \leq \operatorname{int}_{\tilde{\tau} \tilde{\rho} \tilde{\sigma} \tilde{s}}^{\odot}\left(\alpha_{n}, r\right) \leq \alpha_{n} \leq \mathrm{CI}_{\tilde{\tau} \tilde{\tilde{\sigma}} \tilde{\bar{s}} \tilde{\rho}}^{\odot}\left(\alpha_{n}, r\right) \leq \mathrm{C}_{\tilde{\tau} \tilde{\rho} \tilde{\sigma} \tilde{\xi}}\left(\alpha_{n}, r\right)$,

(c) $\mathrm{CI}_{\tilde{\tau} \tilde{\tilde{\sigma}} \tilde{\sigma} \tilde{s}}^{\odot}\left(\left[\alpha_{n}\right]^{c}, r\right)=\left[\operatorname{int}_{\tilde{\tau} \tilde{\tilde{\sigma}} \tilde{\sigma} \tilde{s}}^{\odot}\left(\alpha_{n}, r\right)\right]^{c}$,

(d) $\left[\mathrm{CI}_{\tilde{\tau} \tilde{\rho} \tilde{\sigma} \tilde{s}}^{\odot}\left(\alpha_{n}, r\right)\right]^{c}=\operatorname{int}_{\tilde{\tau} \tilde{\rho} \tilde{\rho} \tilde{s}}^{\odot}\left(\left[\alpha_{n}\right]^{c}, r\right)$,

(e) $\operatorname{int}_{\tilde{\tau} \tilde{\rho} \tilde{\sigma} \tilde{s}}^{\odot}\left(\alpha_{n} \wedge \varepsilon_{n}, r\right)=\operatorname{int}_{\tilde{\tau} \tilde{\varrho} \tilde{\sigma} \tilde{s}}^{\odot}\left(\alpha_{n}, r\right) \wedge \operatorname{int}_{\tilde{\tau} \tilde{\varrho} \tilde{\sigma} \tilde{s}}^{\odot}\left(\varepsilon_{n}, r\right)$.

Definition 12. [8] Let $(\tilde{\mathcal{F}}, \tau \tilde{\varrho} \tilde{\tilde{\sigma}} \tilde{\varsigma})$ be an SVNITS. For every $\alpha_{n}, \varepsilon_{n}, \omega_{n} \in \zeta^{\tilde{\mathcal{F}}}, \alpha_{n}$ and $\varepsilon_{n}$ are called $r$-single-valued neutrosophic separated if for $r \in \zeta_{0}$,

$\mathrm{CI}_{\tau} \tilde{\varrho} \tilde{\sigma} \tilde{\zeta}_{\tilde{s}}\left(\boldsymbol{\alpha}_{\boldsymbol{n}}, \boldsymbol{r}\right) \cap \boldsymbol{\varepsilon}_{\boldsymbol{n}}=\mathbf{C} \mathbf{I}_{\tau \tilde{\tilde{\rho}} \tilde{\tilde{s}} \tilde{\zeta}}\left(\varepsilon_{\boldsymbol{n}}, \boldsymbol{r}\right) \cap \boldsymbol{\alpha}_{\boldsymbol{n}}=\tilde{\mathbf{0}}$ 
An $\mathcal{S} \mathcal{V N S}, \omega_{n}$ is called $r$-single-valued neutrosophic connected if $r$ - $\mathcal{S} \mathcal{N} \mathcal{S E \mathcal { P }} \alpha_{n}, \varepsilon_{n} \in \zeta^{\tilde{\mathcal{F}}}-$ $\{\tilde{0}\}$ such that $\omega_{n}=\alpha_{n} \cup \varepsilon_{n}$ does not exist. A $\mathcal{S} \mathcal{V} \mathcal{N} \mathcal{S} \alpha_{n}$ is said to be $r$-single-valued neutrosophic connected if it is $r$-single-valued neutrosophic connected for any $r \in \zeta_{0}$. A $\left(\tilde{\mathcal{F}}, \tau^{\tilde{\varrho} \tilde{\sigma} \tilde{\zeta}}\right)$ is said to be $r$-single-valued neutrosophic connected if $\tilde{1}$ is $r$-single-valued neutrosophic connected.

\section{Single Valued Neutrosophic $\delta £$-Cluster Point and Single Valued Neutrosophic $\boldsymbol{\theta} £$-Cluster Point}

In this section, we introduce the $r$-single-valued neutrosophic $\delta £$-cluster point (abbreviated $S V N \delta £$-cluster point) and $r$-single-valued neutrosophic $£$-closed set (abbreviated SVN£C). Furthermore, we analyze the single-valued neutrosophic $\delta £$-closure operator ( $\delta £$-closure operator for brevity) and single-valued neutrosophic $\theta £$-closure operator ( $\theta £$-closure operator for brevity).

Definition 13. Let $\left(\tilde{\mathcal{F}}, \tau \tilde{\varrho} \tilde{\sigma} \tilde{\zeta}, £^{\tilde{\varrho} \tilde{\sigma} \tilde{\zeta}}\right)$ be an SVNITS and $\alpha_{n} \in \zeta^{\tilde{\mathcal{F}}}, r \in \zeta_{0}$. Then,

(a) $\alpha_{n}$ is said to be r-single valued neutrosophic $£$-open (briefly, $r-S V N £ O$ ), if and only if $\alpha_{n} \leq \operatorname{int}_{\tilde{\tau} \tilde{\tau} \tilde{\tau} \tilde{\sigma} \tilde{s}}\left(\left[\alpha_{n}\right]_{r}^{\odot}, r\right)$,

(b) $\alpha_{n}$ is said to be r-single valued neutrosophic semi-£-open (briefly, $r$ - $S V N S £ O$ ) if and only if $\alpha_{n} \leq \mathrm{CI}_{\tilde{\tau} \tilde{\varrho} \tilde{\sigma} \tilde{s}}^{\odot}\left(\operatorname{int}_{\tilde{\tau} \tilde{\varrho} \tilde{\sigma} \tilde{\xi}}\left(\left[\alpha_{n}\right]_{r}^{\odot}, r\right), r\right)$,

(c) $\alpha_{n}$ is called r-single valued neutrosophic pre-£-open (briefly, $r-S V N P £ O$ ) if and only if $\alpha_{n} \leq$ $\operatorname{int}_{\tilde{\tau} \tilde{\varrho} \tilde{\sigma} \tilde{s}}\left(\mathrm{CI}_{\tilde{\tau} \tilde{\tau} \tilde{\sigma} \tilde{s}}\left(\left[\alpha_{n}\right]_{r}^{\odot}, r\right), r\right)$,

(d) $\alpha_{n}$ is called $r$-single valued neutrosophic regular-£-open (briefly, $r-S V N R £ O$ ) if and only if $\alpha_{n}=\operatorname{int}_{\tilde{\tau} \tilde{\varrho} \tilde{\sigma} \tilde{s}}\left(\mathrm{CI}_{\tilde{\tau} \tilde{\varrho} \tilde{\sigma} \tilde{s}}^{\odot}\left(\left[\alpha_{n}\right]_{r}^{\odot}, r\right), r\right)$,

(e) $\alpha_{n}$ is said to be r-single valued neutrosophic $\alpha £$-open (briefly, r-SVN $\alpha £ O$ ) if and only if $\alpha_{n} \leq \operatorname{int}_{\tilde{\tau} \tilde{\rho} \tilde{\sigma} \tilde{s}}\left(\mathrm{CI}_{\tilde{\tau} \tilde{\tilde{\sigma}} \tilde{\sigma} \tilde{s}}^{\odot}\left(\operatorname{int}_{\tilde{\tau} \tilde{\varrho}}\left(\left[\alpha_{n}\right]_{r}^{\odot}, r\right), r\right)\right.$,

(f) $\alpha_{n}$ is said to be r-single valued neutrosophic $\star$-open set (briefly, $r-S V N \star \mathrm{O}$ ) if and only if $\alpha_{n}=\mathrm{CI}_{\tilde{\tau} \tilde{\varrho} \tilde{\sigma} \tilde{s}}^{\odot}\left(\alpha_{n}, r\right)$.

The complement of an r-SVN£O (resp, r-SVNS£O, r-SVNP£O, r-SVNR£O, r-SVN $\alpha £ O$, $\mathrm{r}-\mathrm{SVN} \star \mathrm{O}$ ) is said to be an $\mathrm{r}-\mathrm{SVN} £ \mathrm{C}$ (resp, r-SVNS£C, r-SVNP£C, r-SVNR£C, r-SVN $\alpha £ C$, $\mathrm{r}-\mathrm{SVN} \star \mathrm{C}$ ) respectively.

Remark 2. $\mathrm{r}$-single valued neutrosophic open set $(\mathrm{r}-\mathcal{S} \mathcal{V} \mathcal{N O})$ and $\mathrm{r}-\mathrm{SVN} £ \mathrm{O}$ are independent notions as shown by the following example.

Example 1. Let $\tilde{\mathcal{F}}=\{a, b, c\}$ be a set. Define $\varepsilon_{n}, \pi_{n}, \omega_{n} \in \zeta^{\tilde{\mathcal{F}}}$ as follows:

$\varepsilon_{n}=\langle(0.3,0.3,0.3),(0.3,0.3,0.3),(0.3,0.3,0.3)\rangle ; \quad \pi_{n}=\langle(0.4,0.4,0.4),(0.4,0.4,0.4),(0.4,0.4,0.4)\rangle$,

$\omega_{n}=\langle(0.5,0.5,0.5),(0.2,0.2,0.2),(0.1,0.1,0.1)\rangle$.

We define an $\operatorname{SVNITS}\left(\tau^{\tilde{\varrho} \tilde{\sigma} \tilde{\zeta}}, £^{\tilde{\varrho} \tilde{\sigma}} \tilde{\zeta}\right)$ on $\tilde{\mathcal{F}}$ as follows: for each $\alpha_{n} \in \zeta^{\tilde{\mathcal{F}}}$,

$\tilde{\tau}^{\tilde{\varrho}}\left(\alpha_{n}\right)=\left\{\begin{array}{l}1, \quad \text { if } \alpha_{n}=\{\tilde{0}, \tilde{1}\}, \\ \frac{2}{3}, \quad \text { if } \alpha_{n}=\left\{\varepsilon_{n}, \pi_{n}\right\}, \\ 0, \quad \text { otherwise, }\end{array} \quad £^{\tilde{\varrho}}\left(\alpha_{n}\right)= \begin{cases}1, & \text { if } \alpha_{n}=\tilde{0}, \\ \frac{2}{3}, & \text { if } 0<\alpha_{n} \leq \omega_{n} \\ 0, & \text { otherwise, }\end{cases}\right.$ 


$$
\begin{aligned}
& \tilde{\tau}^{\tilde{\sigma}}\left(\alpha_{n}\right)=\left\{\begin{array}{l}
0, \quad \text { if } \alpha_{n}=\{\tilde{0}, \tilde{1}\}, \\
\frac{1}{3}, \quad \text { if } \alpha_{n}=\left\{\varepsilon_{n}, \pi_{n}\right\}, \\
1, \quad \text { otherwise, }
\end{array} \quad £^{\tilde{\sigma}}\left(\alpha_{n}\right)= \begin{cases}0, & \text { if } \alpha_{n}=\tilde{0}, \\
\frac{1}{3}, & \text { if } 0<\alpha_{n} \leq \omega_{n}, \\
1, & \text { otherwise, }\end{cases} \right. \\
& \tilde{\tau}^{\tilde{s}}\left(\alpha_{n}\right)=\left\{\begin{array}{ll}
0, & \text { if } \alpha_{n}=\{\tilde{0}, \tilde{1}\}, \\
\frac{1}{3}, & \text { if } \alpha_{n}=\left\{\varepsilon_{n}, \pi_{n}\right\}, \\
1, & \text { otherwise, }
\end{array} \quad £^{\tilde{s}}\left(\alpha_{n}\right)= \begin{cases}0, & \text { if } \alpha_{n}=\tilde{0}, \\
\frac{1}{3}, & \text { if } 0<\alpha_{n} \leq \omega_{n}, \\
1, & \text { otherwise. }\end{cases} \right.
\end{aligned}
$$

Based on $\varepsilon_{n}=\langle(0.3,0.3,0.3),(0.3,0.3,0.3),(0.3,0.3,0.3)\rangle$, it's clear that, $\frac{2}{3}-\mathcal{S} \mathcal{V N O}$ is set because $\tau^{\tilde{\varrho}}(\langle(0.3,0.3,0.3),(0.3,0.3,0.3),(0.3,0.3,0.3)\rangle) \geq \frac{2}{3}, \tau^{\tilde{\sigma}}(\langle(0.3,0.3,0.3),(0.3,0.3,0.3),(0.3,0.3,0.3)\rangle) \leq$ $\frac{1}{3}, \tau \tilde{s}(\langle(0.3,0.3,0.3),(0.3,0.3,0.3),(0.3,0.3,0.3)\rangle) \leq \frac{1}{3}$.

However $\varepsilon_{\mathrm{n}}$ is not an $r$-SVN£O set, and for that, we must prove that $\varepsilon_{n} \not \leq$ $\operatorname{int}_{\tilde{\tau} \tilde{\varrho} \tilde{\sigma} \tilde{S}}\left(\left[\varepsilon_{n}\right]_{\frac{2}{3}}^{\odot}, \frac{2}{3}\right)$. So, we must first obtain $\left[\varepsilon_{n}\right]_{\frac{2}{3}}^{\odot}$. Based on Eq. (11), $\tilde{1}, \varepsilon_{n}, \pi_{n} \in Q_{\tau} \tilde{\varrho} \tilde{\sigma} \tilde{s}\left(x_{s, t, k}, \frac{2}{3}\right)$ and $£^{\tilde{\varrho}}(\langle(0.5,0.5,0.5),(0.2,0.2,0.2),(0.1,0.1,0.1)\rangle) \geq \frac{2}{3}, £^{\tilde{\sigma}}(\langle(0.5,0.5,0.5),(0.2,0.2,0.2),(0.1,0.1,0.1)\rangle) \leq$ $\frac{1}{3}, £^{\tilde{S}}(\langle(0.5,0.5,0.5),(0.2,0.2,0.2),(0.1,0.1,0.1)\rangle) \leq \frac{1}{3}$, such that by using Eqs. (2), (3) and (6) we obtain, $\tilde{\varrho}_{\varepsilon_{n}}(v)+\tilde{\varrho}_{\tilde{1}}(v)-1>\tilde{\varrho}_{\omega_{n}}(v), \tilde{\sigma}_{\varepsilon_{n}}(v)+\tilde{\sigma}_{\tilde{1}}(v)-1 \leq \tilde{\sigma}_{\omega_{n}}(v), \tilde{\zeta}_{\varepsilon_{n}}(v)+\tilde{\zeta}_{\tilde{1}}(v)-1 \leq \tilde{\zeta}_{\omega_{n}}(v)$.

$(0.3,0.3,0.3)(v)+(1,1,1)(v)-1 \ngtr(0.5,0.5,0.5)(v)$, $(0.3,0.3,0.3)(v)+(0,0,0)(v)-1 \leq(0.2,0.2,0.2)(v)$, $(0.3,0.3,0.3)(v)+(0,0,0)(v)-1 \leq(0.1,0.1,0.1)(v)$, $\tilde{\varrho}_{\varepsilon_{n}}(v)+\tilde{\varrho}_{\pi_{n}}(v)-1>\tilde{\varrho}_{\omega_{n}}(v), \tilde{\sigma}_{\varepsilon_{n}}(v)+\tilde{\sigma}_{\pi_{n}}(v)-1 \leq \tilde{\sigma}_{\omega_{n}}(v), \tilde{\zeta}_{\varepsilon_{n}}(v)+\tilde{\zeta}_{\pi_{n}}(v)-1 \leq \tilde{\zeta}_{\omega_{n}}(v)$. $(0.3,0.3,0.3)(v)+(0.4,0.4,0.4)(v)-1 \ngtr(0.5,0.5,0.5)(v)$, $(0.3,0.3,0.3)(v)+(0.4,0.4,0.4)(v)-1 \leq(0.2,0.2,0.2)(v)$, $(0.3,0.3,0.3)(v)+(0.4,0.4,0.4)(v)-1 \leq(0.1,0.1,0.1)(v)$ $\tilde{\varrho}_{\varepsilon_{n}}(v)+\tilde{\varrho}_{\varepsilon_{n}}(v)-1>\tilde{\varrho}_{\omega_{n}}(v), \tilde{\sigma}_{\varepsilon_{n}}(v)+\tilde{\sigma}_{\varepsilon_{n}}(v)-1 \leq \tilde{\sigma}_{\omega_{n}}(v), \tilde{\zeta}_{\varepsilon_{n}}(v)+\tilde{\zeta}_{\varepsilon_{n}}(v)-1 \leq \tilde{\zeta}_{\omega_{n}}(v)$. $(0.3,0.3,0.3)(v)+(0.3,0.3,0.3)(v)-1 \ngtr(0.5,0.5,0.5)(v)$, $(0.3,0.3,0.3)(v)+(0.3,0.3,0.3)(v)-1 \leq(0.2,0.2,0.2)(v)$, $(0.3,0.3,0.3)(v)+(0.3,0.3,0.3)(v)-1 \leq(0.1,0.1,0.1)(v)$

Therefore, $\left[\varepsilon_{n}\right]_{\frac{2}{3}}^{\odot}=\tilde{0}$. Subsequently, using Eq. (7) we obtain $\operatorname{int}_{\tilde{\tau} \tilde{\tilde{\sigma}} \tilde{s} \tilde{s}}\left(\left[\varepsilon_{n}\right]_{\frac{2}{3}}^{\odot}, \frac{2}{3}\right)=i n t_{\tilde{\tau} \tilde{\sigma} \tilde{\sigma} \tilde{s}}\left(\tilde{0}, \frac{2}{3}\right)=\tilde{0}$, which implies that $\langle(0.3,0.3,0.3),(0.3,0.3,0.3),(0.3,0.3,0.3)\rangle=\varepsilon_{n} \not \operatorname{int}_{\tilde{\tau} \tilde{\varrho} \tilde{\sigma} \tilde{s}}\left(\left[\varepsilon_{n}\right]_{\frac{2}{3}}^{\odot}, \frac{2}{3}\right)=\tilde{0}$. 
Hence, $\varepsilon_{\mathrm{n}}$ is not an $r$-SVN£O set. Then,

Definition 14. Let $\left(\tilde{\mathcal{F}}, \tau^{\tilde{\varrho} \tilde{\sigma} \tilde{\zeta}}, £^{\tilde{\varrho} \tilde{\sigma} \tilde{\zeta}}\right)$ be an SVNITS, $\alpha_{n} \in \zeta^{\tilde{\mathcal{F}}}, x_{s, t, k} \in \mathrm{P}_{s, t, k}(\tilde{\mathcal{F}})$ and $r \in \zeta_{0}$.

(a) $\alpha_{n}$ is an r-single valued neutrosophic $Q_{\tau} \tilde{\varrho} \tilde{\sigma} \tilde{s}$-neighborhood of $x_{s, t, k}$ if $x_{s, t, k} q \alpha_{n}$ with $\tau^{\tilde{\varrho}}\left(\alpha_{n}\right) \geq$ $r, \tau^{\tilde{\sigma}}\left(\alpha_{n}\right) \leq 1-r, \tau^{\tilde{S}}\left(\alpha_{n}\right) \leq 1-r$

(b) $x_{s, t, k}$ is an $\mathrm{r}$-single valued neutrosophic $\theta £$-cluster point ( $\mathrm{r}-\delta £$-cluster point) of $\alpha_{n}$ if for every $\varepsilon_{n} \in Q_{\tilde{\tau} \tilde{\rho} \tilde{\sigma} \tilde{\xi}}\left(x_{s, t, k}, r\right)$, we have $\alpha_{n} q \operatorname{int}_{\tilde{\tau} \tilde{\rho} \tilde{\sigma} \tilde{s}}\left(\mathrm{CI}_{\tilde{\tau} \tilde{\rho} \tilde{\sigma} \tilde{s}}^{\odot}\left(\varepsilon_{n}, r\right), r\right)$

(c) $\delta £$-closure operator is the mapping of $\mathrm{CI}_{\tilde{\tau} \tilde{\varrho} \tilde{\sigma} \tilde{\zeta}}^{\delta £}: \zeta^{\tilde{\mathcal{F}}} \times \zeta_{0} \rightarrow \zeta^{\tilde{\mathcal{F}}}$ defined as $\mathrm{CI}_{\tilde{\tau} \tilde{\varrho} \tilde{\sigma} \tilde{s}}^{\delta \mathcal{E}}\left(\alpha_{n}, r\right)=\cup\left\{x_{s, t, k} \in \mathrm{P}_{s, t, k}(\tilde{\mathcal{F}}): x_{s, t, k}\right.$ is $r-\delta £-$ cluster point of $\left.\alpha_{n}\right\}$.

Definition 15. Let $\left(\tilde{\mathcal{F}}, \tau^{\tilde{\varrho} \tilde{\sigma} \tilde{\zeta}}, £^{\tilde{\varrho} \tilde{\sigma} \tilde{\zeta}}\right)$ be an SVNITS, $\alpha_{n} \in \zeta^{\tilde{\mathcal{F}}}, x_{s, t, k} \in \mathrm{P}_{s, t, k}(\tilde{\mathcal{F}})$ and $r \in \zeta_{0}$. Then,

(a) $\alpha_{n}$ is called r-Single valued neutrosophic $\mathfrak{R}_{\tau \tilde{\varrho} \tilde{\sigma} \tilde{s}}^{\mathfrak{L}}$-neighborhood of $x_{s, t, k}$ if $x_{s, t, k} q \alpha_{n}$ and $\alpha n$ is $r$-SVNRIO. We denote $\mathfrak{R}_{\tau \tilde{\rho} \tilde{\sigma} \tilde{s}}^{\mathfrak{E}}=\left\{\alpha_{n} \in \zeta^{\tilde{\mathcal{F}}} \mid x_{s, t, k} q \alpha_{n}, \alpha_{n}\right.$ is $\left.\mathrm{r}-\mathrm{SVNRIO}\right\}$,

(b) $x_{s, t, k}$ is called $\mathrm{r}$-single valued neutrosophic $\theta £$-cluster point ( $\mathrm{r}-\theta \mathfrak{f}$-cluster point) of $\alpha_{n}$ if for any $\varepsilon_{n} \in Q_{\tilde{\tau} \tilde{\rho} \tilde{\sigma} \tilde{s}}\left(x_{s, t, k}, r\right)$, we have $\alpha_{n} q \mathrm{CI}_{\tilde{\tau} \tilde{\varrho} \tilde{\sigma} \tilde{s}}^{\odot}\left(\varepsilon_{n}, r\right)$,

(c) $\theta \mathfrak{f}$-closure operator is mapping $\mathrm{CI}_{\tilde{\tau} \tilde{\tilde{\alpha}} \tilde{\sigma} \tilde{s}}^{\theta £}: \zeta^{\tilde{\mathcal{F}}} \times \zeta_{0} \rightarrow \zeta^{\tilde{\mathcal{F}}}$ defined as

$$
\mathbf{C I}_{\tilde{\tau} \tilde{\tilde{\sigma} \tilde{s}} \tilde{s}}^{\theta £}\left(\alpha_{n}, r\right)=\cup\left\{x_{s, t, k} \in P_{s, t, k}(\tilde{\mathcal{F}}): x_{s, t, k} \text { is } r-\theta £-\text { cluster point of } \alpha_{n}\right\}
$$

Example 2. Let $\tilde{\mathcal{F}}=\{a, b, c\}$ be a set. Define $\varepsilon_{n}, \pi_{n} \in \zeta^{\tilde{\mathcal{F}}}$ as follows: $\varepsilon_{n}=\langle(0.4,0.4,0.4),(0.4,0.4,0.4),(0.4,0.4,0.4)\rangle ; \pi_{n}=\langle(0.2,0.2,0.2),(0.2,0.2,0.2),(0.2,0.2,0.2)\rangle$.

We define an $\operatorname{SVNITS}\left(\tau^{\tilde{\varrho} \tilde{\sigma} \tilde{\zeta}}, £^{\tilde{\varrho} \tilde{\sigma}} \tilde{\zeta}\right)$ on $\tilde{\mathcal{F}}$ as follows: for each $\alpha_{n} \in \zeta^{\tilde{\mathcal{F}}}$, $\tilde{\tau}^{\tilde{\varrho}}\left(\alpha_{n}\right)=\left\{\begin{array}{ll}1, & \text { if } \alpha_{n}=\tilde{0}, \\ 1, & \text { if } \alpha_{n}=\tilde{1}, \\ \frac{2}{3}, & \text { if } \alpha_{n}=\varepsilon_{n}, \\ 0, & \text { otherwise, }\end{array} \quad £^{\tilde{\varrho}}\left(\alpha_{n}\right)= \begin{cases}1, & \text { if } \alpha_{n}=\tilde{0}, \\ \frac{1}{3}, & \text { if } \pi_{n}=\varepsilon_{n} \\ \frac{2}{3}, & \text { if } 0<\alpha_{n}<\pi_{n} \\ 0, & \text { otherwise, }\end{cases}\right.$ $\tilde{\tau}^{\tilde{\sigma}}\left(\alpha_{n}\right)=\left\{\begin{array}{l}0, \quad \text { if } \alpha_{n}=\tilde{0}, \\ 0, \quad \text { if } \alpha_{n}=\tilde{1}, \\ \frac{1}{3}, \quad \text { if } \alpha_{n}=\varepsilon_{n}, \\ 1, \quad \text { otherwise, }\end{array} \quad \AA^{\tilde{\sigma}}\left(\alpha_{n}\right)= \begin{cases}0, & \text { if } \alpha_{n}=\tilde{0}, \\ \frac{2}{3}, & \text { if } \pi_{n}=\varepsilon_{n} \\ \frac{1}{3}, & \text { if } 0<\alpha_{n}<\pi_{n} \\ 1, & \text { otherwise, }\end{cases}\right.$ 


$$
\tilde{\tau}^{\tilde{s}}\left(\alpha_{n}\right)=\left\{\begin{array}{l}
0, \quad \text { if } \alpha_{n}=\tilde{0}, \\
0, \quad \text { if } \alpha_{n}=\tilde{1}, \\
\frac{1}{3}, \quad \text { if } \alpha_{n}=\varepsilon_{n}, \\
1, \quad \text { otherwise, }
\end{array} \quad £^{\tilde{s}}\left(\alpha_{n}\right)= \begin{cases}0, & \text { if } \alpha_{n}=\tilde{0}, \\
\frac{2}{3}, & \text { if } \pi_{n}=\varepsilon_{n} \\
\frac{1}{3}, & \text { if } 0<\alpha_{n}<\pi_{n} \\
1, & \text { otherwise, }\end{cases}\right.
$$

From using (9) we get, we obtain

$$
\mathrm{CI}_{\tilde{\tau} \tilde{\tau} \tilde{\sigma} \tilde{s}}^{\theta \tilde{E}}\left(\alpha_{n}, r\right)= \begin{cases}\tilde{0}, & \text { if } \alpha_{n}=\tilde{0}, \\ \varepsilon_{n}^{c}, & \text { if } \tilde{0} \neq \alpha_{n} \leq \varepsilon_{n}^{c}, r \leq \frac{1}{3}, 1-r \geq \frac{2}{3}, \\ 1, & \text { otherwise. }\end{cases}
$$

Theorem 3. Let $\left(\tilde{\mathcal{F}}, \tau^{\tilde{\varrho} \tilde{\sigma}} \tilde{\zeta}, £^{\tilde{\varrho} \tilde{\sigma} \tilde{\zeta}}\right)$ be an SVNITS, $r \in \zeta_{0}$ and $\alpha_{n}, \varepsilon_{n} \in \zeta^{\tilde{\mathcal{F}}}$. Then the following properties are holds:

(a) $\alpha_{n} \leq \mathrm{CI}_{\tilde{\tau} \tilde{\varrho} \tilde{\sigma} \tilde{\zeta}}^{\delta £}\left(\alpha_{n}, r\right)$,

(b) If $\alpha_{n} \leq \varepsilon_{n}$, then $\mathrm{CI}_{\tilde{\tau} \tilde{\rho} \tilde{\sigma} \tilde{s}}^{\delta £}\left(\alpha_{n}, r\right) \leq \mathrm{CI}_{\tilde{\tau} \tilde{\varrho} \tilde{\sigma} \tilde{s}}^{\delta \mathcal{E}}\left(\varepsilon_{n}, r\right)$,

(c) $\operatorname{int}_{\tilde{\tau} \tilde{\rho} \tilde{\sigma} \tilde{s}}\left(\mathrm{CI}_{\tilde{\tau} \tilde{\rho} \tilde{\sigma} \tilde{s}}^{\odot}\left(\alpha_{n}, r\right), r\right)$ is $\mathrm{r}-S V N R I O$,

(d) $\mathrm{CI}_{\tilde{\tau} \tilde{\rho} \tilde{\sigma} \tilde{\zeta}}^{\delta £}\left(\alpha_{n}, r\right)=\cap\left\{\varepsilon_{n} \in \zeta^{\tilde{\mathcal{F}}} \mid \alpha_{n} \leq \varepsilon_{n}, \varepsilon_{n}\right.$ is $\left.\mathrm{r}-S V N R I C\right\}$,

(e) $\mathrm{CI}_{\tilde{\tau} \tilde{\rho} \tilde{\sigma} \tilde{\xi}}\left(\alpha_{n}, r\right) \leq \mathrm{CI}_{\tilde{\tau} \tilde{\rho} \tilde{\rho} \tilde{\xi}}^{\delta \mathcal{E}}\left(\alpha_{n}, r\right)$.

Proof. (a) and (b) are easily proved from (9).

(c) Let $\varepsilon_{n} \in \zeta^{\tilde{\mathcal{F}}}$ and $\varepsilon_{n}=\operatorname{int}_{\tilde{\tau} \tilde{\varrho} \tilde{\sigma} \tilde{s}}\left(\mathrm{CI}_{\tilde{\tau} \tilde{\varrho} \tilde{\sigma} \tilde{S}}^{\odot}\left(\alpha_{n}, r\right), r\right)$. Then, we have

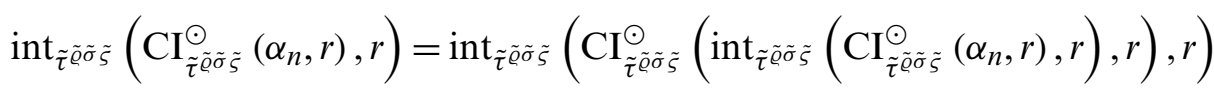

$$
\begin{aligned}
& \leq \operatorname{int}_{\tilde{\tau} \tilde{\rho} \tilde{\sigma} \tilde{s}}\left(\mathrm{CI}_{\tilde{\tau} \tilde{\tilde{\sigma}} \tilde{\sigma} \tilde{s}}^{\odot}\left(\mathrm{CI}_{\tilde{\tau} \tilde{\sigma} \tilde{\sigma} \tilde{s}}^{\odot}\left(\alpha_{n}, r\right), r\right), r\right) \\
& =\operatorname{int}_{\tilde{\tau} \tilde{\rho} \tilde{\sigma} \tilde{s}}\left(\mathrm{CI}_{\tilde{\tau} \tilde{\varrho} \tilde{\rho} \tilde{\sigma}}^{\odot}\left(\alpha_{n}, r\right), r\right)=\varepsilon_{n} .
\end{aligned}
$$

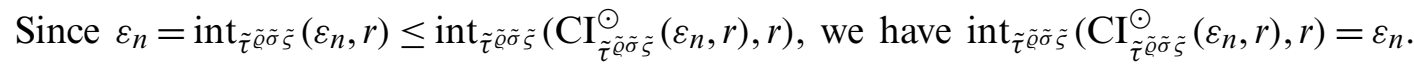

(d) Based on $\mathcal{P}=\cap\left\{\varepsilon_{n} \in \zeta^{\tilde{\mathcal{F}}} \mid \alpha_{n} \leq \varepsilon_{n}, \varepsilon_{n}\right.$ is $r$-SVNRIC\}, let $\mathrm{CI}_{\tilde{\tau} \tilde{\varrho} \tilde{\sigma} \tilde{\xi}}^{\delta \mathcal{E}}\left(\alpha_{n}, r\right) \nsupseteq \mathcal{P}$; therefore, $v \in \tilde{\mathcal{F}}$ and $s \in(0,1], t \in[0,1), k \in[0,1)]$ exist such that

$$
\begin{aligned}
& \tilde{\varrho}_{\mathbf{C I}_{\tilde{\tau} \tilde{\varrho}}^{\delta \varepsilon}\left(\alpha_{n}, r\right)}(v)<s<\tilde{\varrho}_{\mathcal{P}}(v)
\end{aligned}
$$

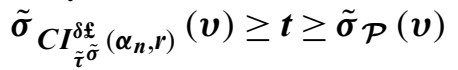

$$
\begin{aligned}
& \tilde{\boldsymbol{S}}_{\boldsymbol{C}}^{\boldsymbol{C}_{\tilde{\tau} \tilde{\boldsymbol{\zeta}}}^{\delta \boldsymbol{f}\left(\alpha_{n}, r\right)}}(\boldsymbol{v}) \geq \boldsymbol{k} \geq \tilde{\boldsymbol{S}}_{\mathcal{P}}(\boldsymbol{v})
\end{aligned}
$$


Therefore, $x_{s, t, k}$ is not an $r$ - $\delta £$-cluster point of $\alpha_{n}$. As such, $\varepsilon_{n} \in Q_{\tilde{\tau} \tilde{\varrho} \tilde{\sigma} \tilde{s}}\left(x_{s, t, k}, r\right)$ and $\alpha_{n} \leq$

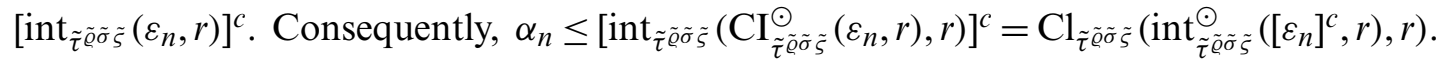

Since $\mathrm{Cl}_{\tilde{\tau} \tilde{\varrho} \tilde{\sigma} \tilde{\zeta}}\left(\operatorname{int}_{\tilde{\tau} \tilde{\varrho} \tilde{\sigma} \tilde{\zeta}}^{\odot}\left(\left[\varepsilon_{n}\right]^{c}, r\right), r\right)$ is $r$-SVNRIC, we have $\tilde{\varrho}_{\mathcal{P}}(v) \leq \tilde{\varrho}_{\left.\mathrm{Cl}_{\tilde{\tau} \tilde{\varrho}} \operatorname{int}_{\tilde{\tau} \tilde{\varrho}}^{\odot}\left(\left[\varepsilon_{n}\right]^{c}, r\right), r\right)}(v)<$

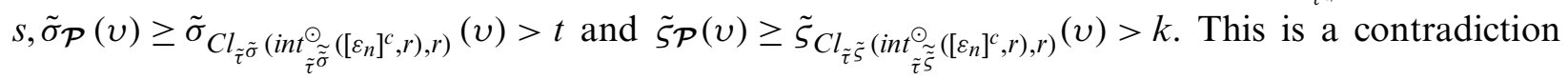
to Eq. (10). Therefore, $\mathrm{CI}_{\tilde{\tau} \tilde{\tilde{\sigma}} \tilde{s} \tilde{s}}^{\delta £}\left(\alpha_{n}, r\right) \geq \mathcal{P}$.

Meanwhile, by setting $\mathrm{CI}_{\tilde{\tau} \tilde{\varrho} \tilde{\sigma} \tilde{\xi}}^{\delta \mathcal{E}}\left(\alpha_{n}, r\right) \not \leq \mathcal{P}$, then an $r$ - $\delta £$-cluster point of $y_{s_{1}, t_{1}, k_{1}} \in P_{s, t, k}(\tilde{\mathcal{F}})$ of $\alpha_{n}$ exists such that

$$
\begin{aligned}
& \tilde{\varrho}_{\mathbf{C I}_{\tilde{\tau} \tilde{\varrho}}^{\delta \varepsilon}\left(\alpha_{n}, r\right)}(y)>s_{1}>\tilde{\varrho}_{\mathcal{P}}(y) \\
& \left.\tilde{\sigma}_{C \tilde{\tau}^{\tilde{\sigma}} \tilde{\delta}\left(\alpha_{n}, r\right)}^{\delta \boldsymbol{E}}(y) \leq \boldsymbol{t}_{1} \leq \tilde{\sigma}_{\mathcal{P}}(y)\right\} \\
& \tilde{\boldsymbol{S}}_{C I_{\tilde{\tilde{\tau}}}^{\delta \mathfrak{\xi}\left(\alpha_{n}, r\right)}}(y) \leq \boldsymbol{k}_{1} \leq \tilde{\boldsymbol{S}}_{\mathcal{P}}(y)
\end{aligned}
$$

Owing to $\mathcal{P}$, there exists $r$-SVNRIC $\varepsilon_{n} \in \zeta^{\tilde{\mathcal{F}}}$ with $\alpha_{n} \leq \varepsilon_{n}$ such that $\tilde{\varrho}_{\mathrm{C}_{\tilde{\tau}}^{\delta \tilde{\varrho}}\left(\alpha_{n}, r\right)}(y)>s_{1}>\tilde{\varrho}_{\varepsilon_{n}} \geq$ $\tilde{\varrho}_{\mathcal{P}}(y), \tilde{\sigma}_{C I_{\tilde{\tau}}^{\delta \tilde{\sigma}}\left(\alpha_{n}, r\right)}(y) \leq t_{1} \leq \tilde{\varrho}_{\varepsilon_{n}} \leq \tilde{\sigma}_{\mathcal{P}}(y)$ and $\tilde{\zeta}_{C I_{\tilde{\tau}}^{\delta \varepsilon}\left(\alpha_{n}, r\right)}(y) \leq k_{1} \leq \tilde{\varrho}_{\varepsilon_{n}} \leq \tilde{\zeta} \mathcal{P}(y)$. Therefore, $\left[\varepsilon_{n}\right]^{c} \in$ $Q_{\tilde{\tau} \tilde{\rho} \tilde{\sigma} \tilde{s}}\left(y_{s_{1}, t_{1}}, k_{1}\right)$. So, $\alpha_{n} \leq \varepsilon_{n}=\left[\operatorname{int}_{\tilde{\tau} \tilde{\rho} \tilde{\sigma} \tilde{s}}\left(\mathrm{Cl}_{\tilde{\tau} \tilde{\tilde{\sigma}} \tilde{\sigma} \tilde{s}}^{\odot}\left(\left[\varepsilon_{n}\right]^{c}, r\right), r\right)\right]^{c}$. Hence, $\alpha_{n} \bar{q} \operatorname{int}_{\tilde{\tau} \tilde{\rho} \tilde{\sigma} \tilde{s}}\left(\mathrm{Cl}_{\tilde{\tau} \tilde{\rho} \tilde{\rho} \tilde{s}}^{\odot}\left(\left[\varepsilon_{n}\right]^{c}, r\right), r\right)$.

Additionally, $y_{s_{1}, t_{1}, k_{1}}$ is not an $r$ - $\delta £$-cluster point of $\alpha_{n}$, that is, $\tilde{\varrho}_{\mathrm{CI}_{\tilde{\tau} \tilde{\varrho}}^{\delta \mathfrak{\varrho}}\left(\alpha_{n}, r\right)}(y)<s_{1}, \tilde{\sigma}_{C I_{\tilde{\tau}}^{\delta \mathfrak{\tilde { \sigma }}}\left(\alpha_{n}, r\right)}(y) \geq$ $t_{1}, \tilde{\zeta}_{C I_{\tilde{\tau} \tilde{\zeta}}^{\delta £}\left(\alpha_{n}, r\right)}(y) \geq k_{1}$. This is a contradiction to Eq. (11). Therefore, $\mathrm{CI}_{\tilde{\tau} \tilde{\varrho} \tilde{\sigma} \tilde{\zeta}}^{\delta \mathcal{E}}\left(\alpha_{n}, r\right) \leq \mathcal{P}$,

(e) Suppose that $\mathrm{Cl}_{\tilde{\tau} \tilde{\rho} \tilde{\sigma} \tilde{\xi}}\left(\alpha_{n}, r\right) \not \leq \mathrm{CI}_{\tilde{\tau} \tilde{\rho} \tilde{\sigma} \tilde{\xi}}^{\delta \mathfrak{E}}\left(\alpha_{n}, r\right)$; therefore, $v \in \tilde{\mathcal{F}}$ and $[s \in(0,1], t \in[0,1), k \in[0,1)]$ exist such that

$$
\begin{aligned}
& \tilde{\varrho}_{\mathbf{C I}_{\tilde{\tau} \tilde{\varrho}}\left(\boldsymbol{\alpha}_{\boldsymbol{n}}, \boldsymbol{r}\right)}(\boldsymbol{v})>\boldsymbol{s}>\tilde{\varrho}_{\mathbf{C I}_{\tilde{\tau} \tilde{\varrho}}^{\delta \varepsilon}\left(\boldsymbol{\alpha}_{n}, \boldsymbol{r}\right)}(\boldsymbol{v}) \\
& \tilde{\boldsymbol{\sigma}}_{C \boldsymbol{I}_{\tilde{\tau} \tilde{\sigma}}\left(\boldsymbol{\alpha}_{n}, \boldsymbol{r}\right)}(\boldsymbol{v}) \leq \boldsymbol{t} \leq \tilde{\boldsymbol{\sigma}}_{\boldsymbol{C}} \boldsymbol{I}_{\tilde{\tau}^{\tilde{\boldsymbol{\sigma}}} \boldsymbol{\delta}\left(\boldsymbol{\alpha}_{n}, \boldsymbol{r}\right)}(\boldsymbol{v}) \\
& \tilde{\boldsymbol{S}}_{C I_{\tilde{\tau} \tilde{\sigma}}\left(\alpha_{n}, r\right)}(v) \leq \boldsymbol{k} \leq \tilde{\boldsymbol{S}}_{C I_{\tilde{\tau} \tilde{\zeta}}^{\delta \mathfrak{\varepsilon}}\left(\alpha_{n}, r\right)}(v),
\end{aligned}
$$

Since, $\tilde{\varrho}_{\mathrm{CI}_{\tilde{\tau} \tilde{\varrho}}\left(\alpha_{n}, r\right)}(v)<s, \tilde{\sigma}_{\mathrm{CI}_{\tilde{\tau} \tilde{\sigma}}\left(\alpha_{n}, r\right)}(v) \geq t, \tilde{\zeta}_{\mathrm{CI}_{\tilde{\tau}} \tilde{\sigma}\left(\alpha_{n}, r\right)}(v) \geq k$, we have $x_{s, t, k}$ not $r$ - $\delta £$-cluster point of $\alpha_{n}$. Therefore, there exists $\varepsilon_{n} \in Q_{\tilde{\tau} \tilde{\varrho} \tilde{\sigma} \tilde{s}}\left(x_{s, t, k}, r\right)$ and $\alpha_{n} \leq\left[\operatorname{int}_{\tilde{\tau} \tilde{\varrho} \tilde{\sigma} \tilde{s}}\left(\mathrm{Cl}_{\tilde{\tau} \tilde{\rho} \tilde{\sigma} \tilde{s}}^{\odot}\left(\varepsilon_{n}, r\right), r\right)\right]^{c}$. Hence, $\tilde{\varrho}_{\mathrm{Cl}_{\tilde{\tau} \tilde{\varrho}}\left(\alpha_{n}, r\right)}(v) \leq \tilde{\varrho}_{\left[\operatorname{int}_{\tilde{\tau} \tilde{\varrho}}\left(\mathrm{Cl}_{\tilde{\tau} \tilde{\varrho}}^{\odot}\left(\varepsilon_{n}, r\right), r\right)\right]^{c}}(v)<s, \quad \tilde{\sigma}_{C l_{\tilde{\tau} \tilde{\sigma}}\left(\alpha_{n}, r\right)}(v) \leq \tilde{\varrho}_{\left[i n t_{\tilde{\tau} \tilde{\sigma}}\left(C l_{\tilde{\tau} \tilde{\sigma}}\left(\varepsilon_{n}, r\right), r\right)\right]^{c}}(v) \geq t$ and $\left.\tilde{\zeta} C l_{\tilde{\tau} \tilde{\zeta}}\left(\alpha_{n}, r\right)(v) \leq \tilde{\varrho}_{\left[i n t_{\tilde{\tau} \tilde{S}}\right.}\left(C l_{\tilde{\tau} \tilde{\zeta}}^{\odot}\left(\varepsilon_{n}, r\right), r\right)\right]^{c}(v) \geq k$. It is a contradiction for Eq. (12). Thus $\mathrm{Cl}_{\tilde{\tau} \tilde{\varrho} \tilde{\sigma} \tilde{\zeta}}\left(\alpha_{n}, r\right) \leq$ $\mathrm{CI}_{\tilde{\tau} \tilde{\rho} \tilde{\rho} \tilde{s}}^{\delta £}\left(\alpha_{n}, r\right)$.

Theorem 4. Let $\left(\tilde{\mathcal{F}}, \tau^{\tilde{\varrho} \tilde{\sigma} \tilde{\zeta}}, £^{\tilde{\varrho} \tilde{\sigma} \tilde{\zeta}}\right)$ be an SVNITS, for each $r \in \zeta_{0}$ and $\alpha_{n}, \varepsilon_{n} \in \zeta^{\tilde{\mathcal{F}}}$. Then the following properties hold:

(a) $\alpha_{\mathrm{n}} \leq \mathrm{CI}_{\tilde{\tau} \tilde{\varrho} \tilde{\sigma} \tilde{\zeta}}^{\theta \mathcal{E}}\left(\alpha_{\mathrm{n}}, \mathrm{r}\right)$,

(b) If $\alpha_{\mathrm{n}} \leq \varepsilon_{\mathrm{n}}$, then $\mathrm{CI}_{\tilde{\tau} \tilde{\rho} \tilde{\sigma} \tilde{\sigma}}^{\theta £}\left(\alpha_{\mathrm{n}}, \mathrm{r}\right) \leq \mathrm{CI}_{\tilde{\tau} \tilde{\rho} \tilde{\sigma} \tilde{\zeta}}^{\theta \mathcal{E}}\left(\varepsilon_{\mathrm{n}}, \mathrm{r}\right)$,

(c) $\mathrm{CI}_{\tilde{\tau} \tilde{\varrho} \tilde{\sigma} \tilde{s}}\left(\alpha_{\mathrm{n}}, \mathrm{r}\right) \leq \cup\left\{\mathrm{x}_{\mathrm{s}, \mathrm{t}, \mathrm{k}} \in \mathrm{P}_{\mathrm{s}, \mathrm{t}, \mathrm{k}}(\tilde{\mathcal{F}}) \mid \mathrm{x}_{\mathrm{s}, \mathrm{t}, \mathrm{k}}\right.$ is $\mathrm{r}$ - $\delta £$-cluster point of $\left.\alpha_{\mathrm{n}}\right\}$, 
(d) $\operatorname{CI}_{\tilde{\tau} \tilde{\varrho} \tilde{\sigma} \tilde{s}}^{\ominus \mathcal{E}}\left(\alpha_{\mathrm{n}}, \mathrm{r}\right)=\cap\left\{\varepsilon_{\mathrm{n}} \in \zeta^{\tilde{\mathcal{F}}} \mid \alpha_{\mathrm{n}} \leq \mathrm{int}_{\tilde{\tau} \tilde{\varrho} \tilde{\sigma} \tilde{\xi}}^{\odot}\left(\varepsilon_{\mathrm{n}}, \mathrm{r}\right), \tau^{\tilde{\varrho}}\left(\left[\varepsilon_{\mathrm{n}}\right]^{\mathrm{c}}\right) \geq \mathrm{r}, \tau^{\tilde{\sigma}}\left(\left[\varepsilon_{\mathrm{n}}\right]^{\mathrm{c}}\right) \leq 1-\mathrm{r}, \tau^{\tilde{s}}\left(\left[\varepsilon_{\mathrm{n}}\right]^{\mathrm{c}}\right) \leq 1-\mathrm{r}\right\}$,

(e) $\mathrm{CI}_{\tilde{\tau} \tilde{\varrho} \tilde{\rho} \tilde{\xi}}^{\delta \mathcal{E}}\left(\alpha_{\mathrm{n}}, \mathrm{r}\right)=\cap\left\{\varepsilon_{\mathrm{n}} \in \zeta^{\tilde{\mathcal{F}}} \mid \alpha_{\mathrm{n}} \leq \varepsilon_{\mathrm{n}}, \varepsilon_{\mathrm{n}}\right.$ is $\mathrm{r}$ - $\delta \mathcal{E}$-cluster point of $\left.\alpha_{\mathrm{n}}\right\}$

(f) $\mathrm{x}_{\mathrm{s}, \mathrm{t}, \mathrm{k}}$ is $\mathrm{r}$ - $\theta £$-cluster point of $\alpha_{\mathrm{n}}$ iff $\mathrm{x}_{\mathrm{s}, \mathrm{t}, \mathrm{k}} \in \mathrm{CI}_{\tilde{\tau} \boldsymbol{\rho} \tilde{\sigma} \tilde{\zeta}}^{\theta £}\left(\alpha_{\mathrm{n}}, \mathrm{r}\right)$,

(g) $\mathrm{x}_{\mathrm{s}, \mathrm{t}, \mathrm{k}}$ is $\mathrm{r}$ - $\delta £$-cluster point of $\alpha_{\mathrm{n}}$ iff $\mathrm{x}_{\mathrm{s}, \mathrm{t}, \mathrm{k}} \in \mathrm{CI}_{\tilde{\tau} \tilde{\varrho} \tilde{\sigma} \tilde{\zeta}}^{\delta \mathfrak{f}}\left(\alpha_{\mathrm{n}}, \mathrm{r}\right)$,

(h) If $\alpha_{\mathrm{n}}=\mathrm{Cl}_{\tilde{\tau} \tilde{\varrho} \tilde{\sigma} \tilde{\xi}}\left(\operatorname{intl}_{\tilde{\tau} \tilde{\rho} \tilde{\sigma} \tilde{s}}^{\odot}\left(\alpha_{\mathrm{n}}, \mathrm{r}\right), \mathrm{r}\right)$, then $\mathrm{CI}_{\tilde{\tau} \tilde{\tilde{\sigma}} \tilde{\bar{s}} \tilde{\mathrm{f}}}^{\delta \tilde{E}}\left(\alpha_{\mathrm{n}}, \mathrm{r}\right)=\alpha_{\mathrm{n}}$,

(i) $\alpha_{\mathrm{n}} \leq \mathrm{CI}_{\tilde{\tau} \tilde{\varrho} \tilde{\sigma} \tilde{s}}\left(\alpha_{\mathrm{n}}, \mathrm{r}\right) \leq \mathrm{CI}_{\tilde{\tau} \tilde{\rho} \tilde{\sigma} \tilde{s}}^{\delta \mathcal{E}}\left(\alpha_{\mathrm{n}}, \mathrm{r}\right) \leq \mathrm{CI}_{\tilde{\tau} \tilde{\sigma} \tilde{\sigma} \tilde{s}}^{\theta \tilde{E}}\left(\alpha_{\mathrm{n}}, \mathrm{r}\right)$,

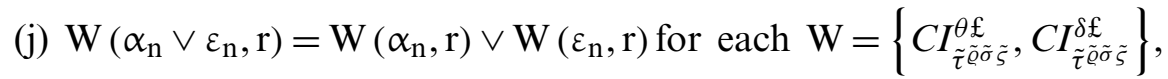

(k) $\mathrm{CI}_{\tilde{\tau} \tilde{\varrho} \tilde{\sigma} \tilde{\zeta}}^{\delta \delta}\left(\mathrm{CI}_{\tilde{\tau} \tilde{\rho} \tilde{\sigma} \tilde{\zeta}}^{\delta \mathcal{E}}\left(\alpha_{\mathrm{n}}, \mathrm{r}\right), \mathrm{r}\right)=\mathrm{CI}_{\tilde{\tau} \tilde{\rho} \tilde{\sigma} \tilde{\zeta}}^{\delta \mathcal{E}}\left(\alpha_{\mathrm{n}}, \mathrm{r}\right)$.

Proof. (a) and (b) are easily proved from Definition 14.

(c) Set $\mathcal{P}=\cup\left\{x_{s, t, k} \in P_{s, t, k}(\tilde{\mathcal{F}}) \mid x_{s, t, k}\right.$ as an $r$ - $\delta \mathfrak{f}$-cluster point of $\left.\alpha_{n}\right\}$. Suppose that $\mathrm{CI}_{\tilde{\tau} \tilde{\rho} \tilde{\sigma} \tilde{s}}\left(\alpha_{n}, r\right) \not \leq \mathcal{P}$. Then there exists $v \in \tilde{\mathcal{F}}$, and $[s \in(0,1], t \in[0,1), k \in[0,1)]$ such that

$\left.\begin{array}{l}\tilde{\varrho}_{C_{\tilde{\tau} \tilde{\varrho}}\left(\alpha_{n}, r\right)}(v)>\boldsymbol{s}>\tilde{\varrho}_{\mathcal{P}}(v) \\ \tilde{\sigma}_{C I_{\tilde{\tau} \tilde{\sigma}}\left(\alpha_{n}, r\right)}(v) \leq \boldsymbol{t} \leq \tilde{\boldsymbol{\sigma}}_{\mathcal{P}}(v) \\ \tilde{\boldsymbol{S}}_{C I_{\tilde{\tau} \tilde{\boldsymbol{\zeta}}}\left(\alpha_{n}, r\right)}(v) \leq \boldsymbol{k} \leq \tilde{\boldsymbol{S}}_{\mathcal{P}}(\boldsymbol{v})\end{array}\right\}$

Consequently, $x_{s, t, k}$ is not $r$ - $\delta £$-cluster point of $\alpha_{n}$. So, there exists $\varepsilon_{n} \in Q_{\tau} \tilde{\varrho} \tilde{\sigma} \tilde{\xi}\left(x_{s, t, k}, r\right)$ and $\alpha_{n} \leq\left[\operatorname{int}_{\tilde{\tau} \tilde{\varrho} \tilde{\sigma} \tilde{s}}\left(\mathrm{CI}_{\tilde{\tau} \tilde{\varrho} \tilde{\sigma} \tilde{s}}^{\odot}\left(\varepsilon_{n}, r\right), r\right)\right]^{c} \leq\left[\varepsilon_{n}\right]^{c}$

Based on Eq. (4), $\tilde{\varrho}_{\mathrm{CI}_{\tilde{\tau} \tilde{\varrho}}\left(\alpha_{n}, r\right)}(v) \leq \tilde{\varrho}_{\left[\varepsilon_{n}\right]^{c}}(v)<s, \tilde{\sigma}_{C I_{\tilde{\tau} \tilde{\sigma}}\left(\alpha_{n}, r\right)}(v) \geq$ $\tilde{\sigma}_{\left[\varepsilon_{n}\right]^{c}}(v) \geq t$ and $\tilde{\zeta}_{C I_{\tilde{\tau}} \tilde{\zeta}}\left(\alpha_{n}, r\right)(v) \geq \tilde{\zeta}_{\left[\varepsilon_{n}\right]^{c}}(v) \geq k$.

It is a contradiction for Eq. (13). Thus $\mathrm{CI}_{\tilde{\tau} \tilde{\rho} \tilde{\sigma} \tilde{\zeta}}\left(\alpha_{n}, r\right) \leq \mathcal{P}$.

(d) $\gamma=\cap\left\{\varepsilon_{n} \in \zeta^{\tilde{\mathcal{F}}} \mid \alpha_{n} \leq \operatorname{int}_{\tilde{\tau} \tilde{\varrho} \tilde{\sigma} \tilde{\zeta}}^{\odot}\left(\varepsilon_{n}, r\right), \tau^{\tilde{\varrho}}\left(\left[\varepsilon_{n}\right]^{c}\right) \geq r, \tau^{\tilde{\sigma}}\left(\left[\varepsilon_{n}\right]^{c}\right) \leq 1-r, \tau^{\tilde{\zeta}}\left(\left[\varepsilon_{n}\right]^{c}\right) \leq 1-r\right\}$.

Suppose that $\mathrm{CI}_{\tilde{\tau} \tilde{\tilde{\sigma}} \tilde{s} \tilde{s}}^{\theta £}\left(\alpha_{n}, r\right) \nsupseteq \gamma$, then there exists $v \in \tilde{\mathcal{F}}$ and $[s \in(0,1], t \in[0,1), k \in[0,1)]$ such that

$$
\begin{aligned}
& \left.\tilde{\varrho}_{\mathbf{C I}}{\underset{\tilde{\tau}}{\theta \tilde{\sigma} \tilde{\sigma} \tilde{s}}\left(\boldsymbol{\alpha}_{\boldsymbol{n}}, \boldsymbol{r}\right)}(\boldsymbol{v})<\boldsymbol{s} \leq \tilde{\boldsymbol{Q}} \boldsymbol{\gamma}(\boldsymbol{v})\right) \\
& \tilde{\boldsymbol{\sigma}}_{C \boldsymbol{C}_{\tilde{\tau} \tilde{\tilde{\sigma}}}^{\theta \boldsymbol{\varepsilon}}\left(\boldsymbol{\alpha}_{\boldsymbol{n}}, \boldsymbol{r}\right)}(\boldsymbol{v})>\boldsymbol{t} \geq \tilde{\boldsymbol{\sigma}} \boldsymbol{\gamma}(\boldsymbol{v}) \\
& \tilde{\boldsymbol{S}}_{C I_{\tilde{\tau} \tilde{\zeta}}^{\theta \varepsilon}\left(\alpha_{n}, r\right)}(v)>k \geq \tilde{\zeta} \gamma(v)
\end{aligned}
$$

Consequently, $x_{s, t, k}$ is not $r-\theta £$-cluster point of $\alpha_{n}$. So, there exists $\varepsilon_{n} \in Q_{\tau \tilde{\varrho} \tilde{\sigma} \tilde{\xi}}\left(x_{s, t, k}, r\right), \alpha_{n} \leq$ $\left[\left(\mathrm{Cl}_{\tilde{\tau} \tilde{\varrho} \tilde{\rho} \tilde{\sigma}}^{\odot}\left(\varepsilon_{n}, r\right), r\right)\right]^{c}$. Thus, $\alpha_{n} \leq\left[\left(\mathrm{Cl}_{\tilde{\tau} \tilde{\tilde{\rho} \tilde{\sigma} \tilde{\zeta}}}^{\odot}\left(\varepsilon_{n}, r\right), r\right)\right]^{c}=\left(\mathrm{int}_{\tilde{\tau} \tilde{\tilde{\rho} \tilde{\sigma} \tilde{\zeta}}}^{\odot}\left(\left[\varepsilon_{n}\right]^{c}, r\right), r\right), \tau^{\tilde{\varrho}}\left(\varepsilon_{n}\right) \geq r, \tau^{\tilde{\sigma}}\left(\varepsilon_{n}\right) \leq 1-r$, $\left.\tau^{\tilde{\zeta}}\left(\varepsilon_{n}\right) \leq 1-r\right\}$. Hence, $\tilde{\varrho}_{\gamma}(v) \leq \tilde{\varrho}_{\left[\varepsilon_{n}\right]^{c}}(v)<s, \tilde{\sigma}_{\gamma}(v) \leq \tilde{\sigma}_{\left[\varepsilon_{n}\right]^{c}}(v)<t, \tilde{\zeta}_{\gamma}(v) \leq \tilde{\zeta}_{\left[\varepsilon_{n}\right]^{c}}(v)<k$.

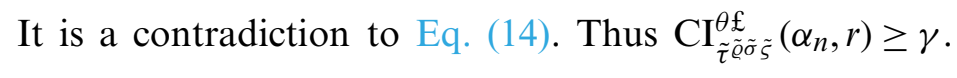




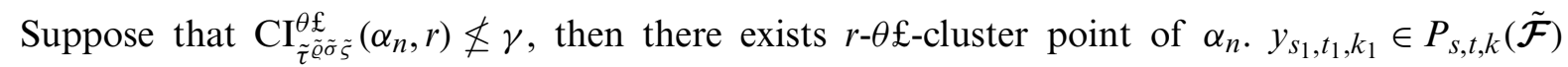
of $\alpha_{n}$, such that

$$
\begin{aligned}
& \tilde{\varrho}_{\mathbf{C I}_{\tilde{\tau} \tilde{\tilde{Q}}}^{\theta \in\left(\alpha_{n}, r\right)}}(y)>s_{1}>\tilde{\varrho}_{\gamma}(y) \\
& \left.\tilde{\sigma}_{C I_{\tilde{\tau}}^{\theta \tilde{\tilde{\sigma}}}\left(\alpha_{n}, r\right)}(y)<\boldsymbol{t}_{1} \leq \tilde{\sigma}_{\gamma}(y)\right\} \\
& \left.\tilde{\boldsymbol{S}}_{C I_{\tilde{\tau} \tilde{\tilde{S}}}^{\theta \underline{\varepsilon}}\left(\alpha_{n}, r\right)}(y)<\boldsymbol{k}_{1} \leq \tilde{\boldsymbol{S}}_{\gamma}(y)\right\}
\end{aligned}
$$

By the definition of $\gamma$, there exists $\varepsilon_{n} \in \zeta^{\tilde{\mathcal{F}}}$ with $\tau^{\tilde{\varrho}}\left(\varepsilon_{n}\right) \geq r, \tau^{\tilde{\sigma}}\left(\varepsilon_{n}\right) \leq 1-r, \tau^{\tilde{\zeta}}\left(\varepsilon_{n}\right) \leq 1-r$

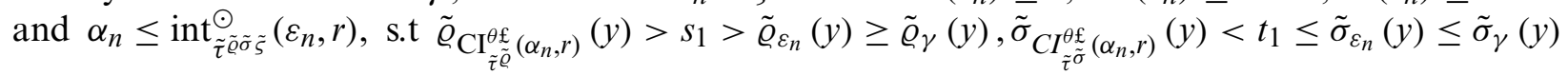
and $\tilde{\zeta}_{C I_{\tilde{\tau} \tilde{\zeta}}^{\theta \varepsilon}\left(\alpha_{n}, r\right)}(y)<k_{1} \leq \tilde{\zeta}_{\varepsilon_{n}}(y) \leq \tilde{\zeta}_{\gamma}(y)$. Additionally, $\left[\varepsilon_{n}\right]^{c} \in Q_{\tau} \tilde{\varrho} \tilde{\sigma} \tilde{\zeta}\left(y_{s_{1}, t_{1}, k_{1}}, r\right) . \alpha_{n} \leq \operatorname{int}_{\tilde{\tau} \tilde{\varrho} \tilde{\sigma} \tilde{\zeta}}^{\odot}\left(\varepsilon_{n}, r\right)=$ $\left[\mathrm{Cl}_{\tilde{\tau} \tilde{\tilde{\sigma}} \tilde{\sigma} \tilde{s}}^{\odot}\left(\left[\varepsilon_{n}\right]^{c}, r\right)\right]^{c}$, implies $\alpha_{n} \bar{q} \mathrm{Cl}_{\tilde{\tau} \tilde{\rho} \tilde{\sigma} \tilde{\xi}}^{\odot}\left(\left[\varepsilon_{n}\right]^{c}, r\right)$. Hence $y_{s_{1}, t_{1}, k_{1}}$ is not an $r-\theta £$-cluster point of $\alpha_{n}$. It is

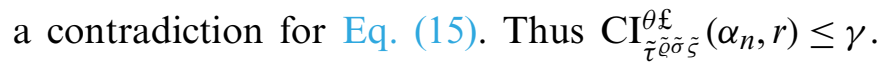

(e) Similar results are shown in (c) and (d).

(f) $(\Rightarrow)$, clear.

$(\Leftarrow)$ Suppose that $x_{s, t, k}$ is not an $r-\theta £$-cluster point of $\alpha_{n}$. There exists $\varepsilon_{n} \in Q_{\tau} \tilde{\varrho} \tilde{\sigma} \tilde{\xi}\left(x_{s, t, k}, r\right)$ such that $\mathrm{Cl}_{\tilde{\tau} \tilde{\varrho} \tilde{\sigma} \tilde{\xi}}^{\odot}\left(\varepsilon_{n}, r\right) \leq \alpha_{n}$. Thus $\alpha_{n} \leq\left[\mathrm{Cl}_{\tilde{\tau} \tilde{\varrho} \tilde{\sigma} \tilde{\xi}}^{\odot}\left(\varepsilon_{n}, r\right)\right]^{c}=\mathrm{Cl}_{\tilde{\tau} \tilde{\varrho} \tilde{\sigma} \tilde{\xi}}^{\odot}\left(\left[\varepsilon_{n}\right]^{c}, r\right)$. By $(\mathrm{d}), \tilde{\varrho}_{\mathrm{Cl}_{\tilde{\tau}}^{\theta \varepsilon}\left(\alpha_{n}, r\right)}(v) \leq \tilde{\varrho}_{\left[\varepsilon_{n}\right]^{c}}(v)<$

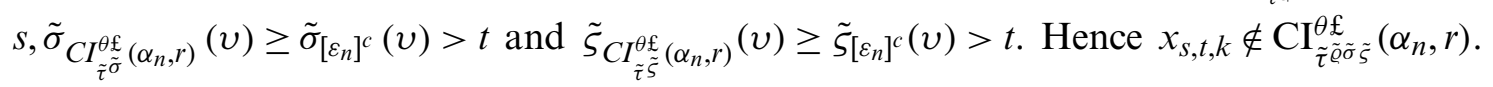

(g) is similarly proved as in (f).

(h) The validity of this axiom is obvious from Theorem 3 (4).

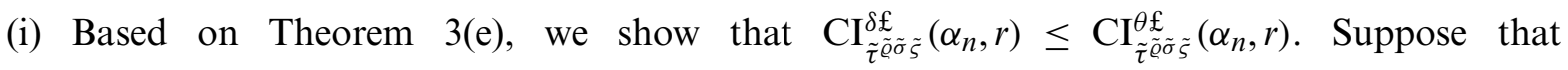
$\mathrm{CI}_{\tilde{\tau} \tilde{\rho} \tilde{\sigma} \tilde{\zeta}}^{\delta £}\left(\alpha_{n}, r\right) \not \leq \mathrm{CI}_{\tilde{\tau} \tilde{\varrho} \tilde{\sigma} \tilde{\zeta}}^{\theta \tilde{E}}\left(\alpha_{n}, r\right)$, then there exists $v \in \zeta$ and $[s \in(0,1], t \in[0,1), k \in[0,1)]$ such that

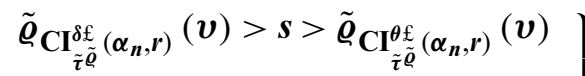

$$
\begin{aligned}
& \tilde{\sigma}_{C I_{\tilde{\tau}}^{\delta \boldsymbol{\sigma}}\left(\alpha_{n}, r\right)}(v)>\boldsymbol{t} \geq \tilde{\sigma}_{C I_{\tilde{\tau}}^{\theta \dot{\boldsymbol{\sigma}}\left(\alpha_{n}, r\right)}}(\boldsymbol{v}) \\
& \tilde{S}_{C I_{\tilde{\zeta}}^{\delta \mathfrak{E}}\left(\alpha_{n}, r\right)}(v)>k \geq \tilde{S}_{C I_{\tilde{\tau} \tilde{\tilde{S}}}^{\theta \ell}\left(\alpha_{n}, r\right)}(v)
\end{aligned}
$$

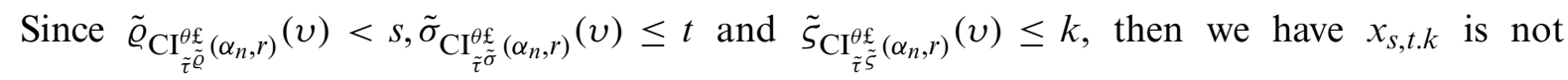

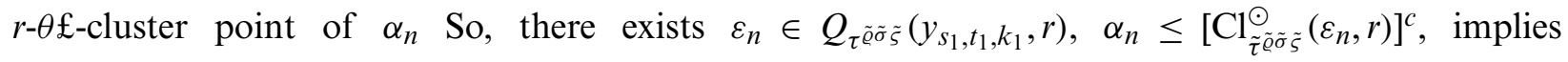
$A \bar{q} \operatorname{int}_{\tilde{\tau} \tilde{\rho} \tilde{\sigma} \tilde{\xi}}\left(\mathrm{Cl}_{\tilde{\tau} \tilde{\tilde{\sigma}} \tilde{\sigma} \tilde{s}}^{\odot}\left(\varepsilon_{n}, r\right), r\right)$. Hence, $x_{s, t, k}$ is not $r-\delta £$-cluster point of $\alpha_{n}$, by (7), we can get than, $\tilde{\varrho}_{\mathrm{CI}_{\tilde{\tau} \tilde{\tilde{\varrho}}}^{\delta \mathcal{E}}\left(\alpha_{n}, r\right)}(v)<s, \sigma_{\mathrm{Cl}_{\tilde{\tau}}^{\delta \mathcal{\sigma}}\left(\alpha_{n}, r\right)}(v) \geq t, \tilde{\zeta}_{\mathrm{CI}_{\tilde{\tau} \tilde{\tilde{s}}}^{\delta \mathcal{E}}\left(\alpha_{n}, r\right)}(v) \geq k$. It is a contradiction for Eq. (16). Thus,

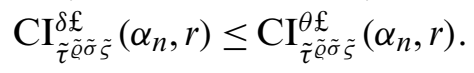




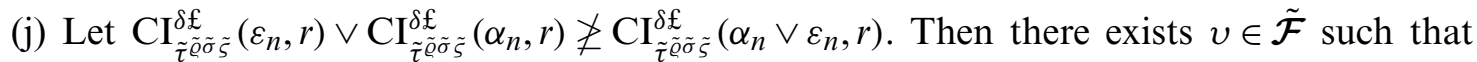

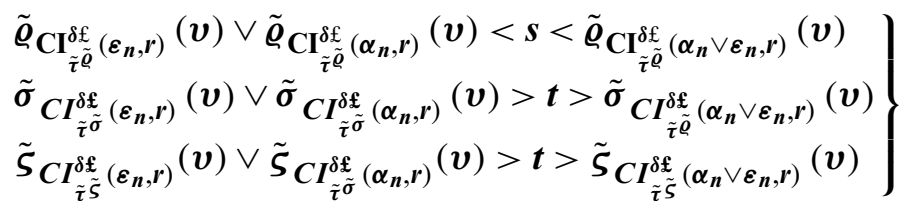

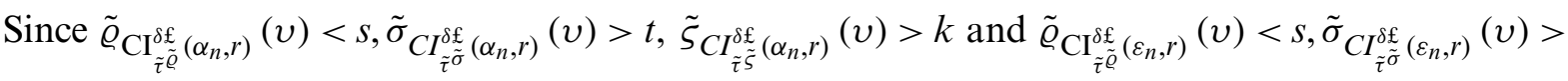
$t, \tilde{\zeta}_{C \tilde{\tau}_{\tilde{\xi}}^{\delta £}\left(\varepsilon_{n}, r\right)}(v)>k$. We obtain, $x_{s, t, k}$ is not $r$ - $\delta £$-cluster point of $\alpha_{n}$ and $\varepsilon_{n}$ So, there exists

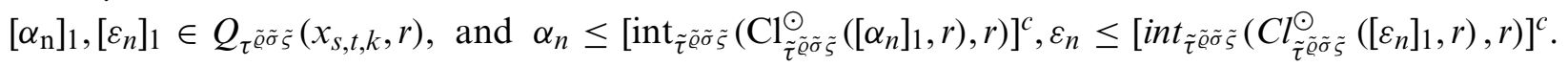
Thus, $\left[\alpha_{n}\right]_{1} \wedge\left[\varepsilon_{n}\right]_{1} \in Q_{\tau} \tilde{\varrho} \tilde{\sigma} \tilde{S}\left(x_{s, t, k}, r\right)$.

Using Eqs. (4) and (5) we obtain,

$$
\begin{aligned}
& \alpha_{n} \vee \varepsilon_{n} \leq\left[\operatorname{int}_{\tilde{\tau} \tilde{\varrho} \tilde{\sigma} \tilde{s}}\left(\mathrm{Cl}_{\tilde{\tau} \tilde{\rho} \tilde{\sigma} \tilde{s}}^{\odot}\left(\left[\alpha_{n}\right]_{1}, r\right), r\right) \wedge \operatorname{int}_{\tilde{\tau} \tilde{\varrho} \tilde{\sigma} \tilde{s}}\left(C l_{\tilde{\tau} \tilde{\rho} \tilde{\sigma} \tilde{s}}^{\odot}\left(\left[\varepsilon_{n}\right]_{1}, r\right), r\right)\right]^{c} \\
& \leq\left[\operatorname{int}_{\tilde{\tau} \tilde{\rho} \tilde{\sigma} \tilde{\zeta}}\left(\mathrm{Cl}_{\tilde{\tau} \tilde{\varrho} \tilde{\sigma} \tilde{\zeta}}^{\odot}\left(\left[\alpha_{n}\right]_{1}, r\right) \wedge \mathrm{Cl}_{\tilde{\tau} \tilde{\varrho} \tilde{\sigma} \tilde{\zeta}}^{\odot}\left(\left[\varepsilon_{n}\right]_{1}, r\right), r\right)\right]^{c} \\
& \leq\left[\operatorname{int}_{\tilde{\tau} \tilde{\varrho} \tilde{\sigma} \tilde{\zeta}}\left(\mathrm{Cl}_{\tilde{\tau} \tilde{\varrho} \tilde{\sigma} \tilde{\zeta}}^{\odot}\left(\left[\alpha_{n}\right]_{1} \wedge\left[\varepsilon_{n}\right]_{1}, r\right), r\right)\right]^{c} .
\end{aligned}
$$

Therefore, $\alpha_{n} \vee \varepsilon_{n} \bar{q} \operatorname{int}_{\tilde{\tau} \tilde{\varrho} \tilde{\sigma} \tilde{\zeta}}\left(\mathrm{Cl}_{\tilde{\tau} \tilde{\varrho} \tilde{\sigma} \tilde{\zeta}}^{\odot}\left(\left[\alpha_{n}\right]_{1} \wedge\left[\varepsilon_{n}\right]_{1}, r\right), r\right)$. Hence, $x_{s, t, k}$ is not $r$ - $\delta £$-cluster point

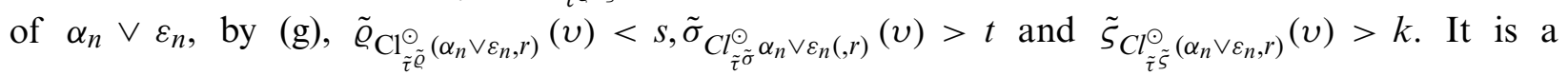

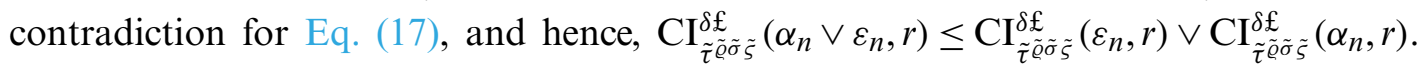

Meanwhile, $\alpha_{n} \vee \varepsilon_{n} \geq \alpha_{n}$ and $\alpha_{n} \vee \varepsilon_{n} \geq \varepsilon_{n}$. Hence $\mathrm{CI}_{\tilde{\tau} \tilde{\rho} \tilde{\sigma} \tilde{s}}^{\delta \mathcal{E}}\left(\alpha_{n} \vee \varepsilon_{n}, r\right) \geq \mathrm{CI}_{\tilde{\tau} \tilde{\alpha} \tilde{\sigma} \tilde{s}}^{\delta £}\left(\varepsilon_{n}, r\right) \vee$

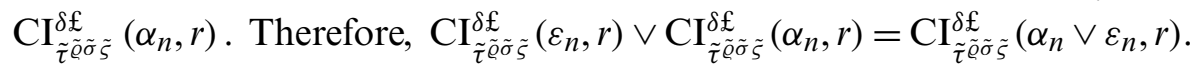

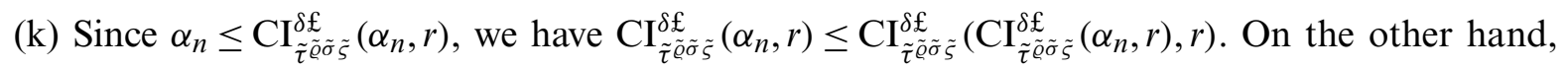

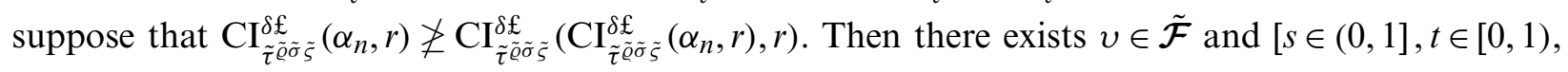
$k \in[0,1)]$ such that

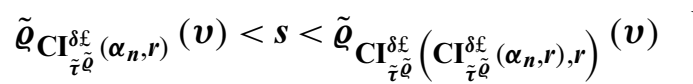

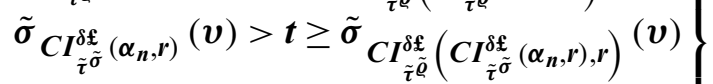

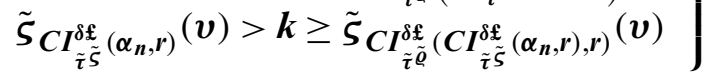

Since $\tilde{\varrho}_{\mathrm{CI}_{\tilde{\tau} \tilde{\tilde{Q}}}^{\delta \mathcal{E}}\left(\alpha_{n}, r\right)}(v)<s, \tilde{\sigma}_{\mathrm{C}_{\tilde{\tau} \tilde{\tilde{\sigma}}}^{\delta \mathfrak{E}}\left(\alpha_{n}, r\right)}(v)>t, \tilde{\zeta}_{\mathrm{CI}_{\tilde{\tau} \tilde{\tilde{\zeta}}}^{\delta \mathfrak{f}}\left(\alpha_{n}, r\right)}(v)>k$, we have $x_{s, t, k}$ is not an $r-\delta £-$ cluster point of $\alpha_{n}$. So, there exists $\varepsilon_{n} \in Q_{\tau \tilde{\rho} \tilde{\sigma} \tilde{\zeta}}\left(x_{s, t, k}, r\right)$ such that $\alpha_{n} \leq\left[\operatorname{int}_{\tilde{\tau} \tilde{\rho} \tilde{\sigma} \tilde{\zeta}}\left(\mathrm{Cl}_{\tilde{\tau} \tilde{\rho} \tilde{\rho} \tilde{\sigma}}^{\odot}\left(\varepsilon_{n, r), r)}\right]^{c}=\right.\right.$ $\mathrm{Cl}_{\tilde{\tau} \tilde{\rho} \tilde{\sigma} \tilde{\xi}}\left(\operatorname{int}_{\tilde{\tau} \tilde{\sigma} \tilde{\sigma} \tilde{s}}^{\odot}\left(\varepsilon_{n}, r, r\right)\right.$, since, $\mathrm{Cl}_{\tilde{\tau} \tilde{\rho} \tilde{\sigma} \tilde{\xi}}\left(\mathrm{int}_{\tilde{\tau} \boldsymbol{\rho} \tilde{\sigma} \tilde{s}}^{\odot}\left(\varepsilon_{n}, r, r\right)\right.$ is $\mathrm{r}-\mathrm{SVNRIC}$. Then by Theorem 3(d), $\mathrm{CI}_{\tilde{\tau} \tilde{\rho} \tilde{\sigma} \tilde{s}}^{\delta \mathcal{E}}\left(\alpha_{n}, r\right) \leq \mathrm{Cl}_{\tilde{\tau} \tilde{\rho} \tilde{\sigma} \tilde{\xi}}\left(\operatorname{int}_{\tilde{\tau} \tilde{\rho} \tilde{\sigma} \tilde{s}}^{\odot}\left(\varepsilon_{n}, r, r\right)\right.$.

Hence,

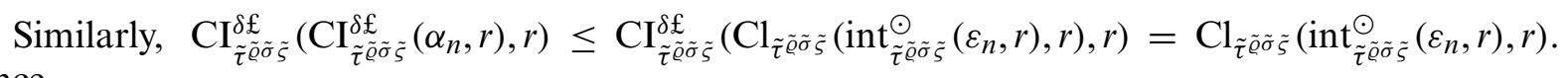


$\mathrm{CI}_{\tilde{\tau} \tilde{\rho} \tilde{\sigma} \tilde{\xi}}^{\delta \mathcal{E}}\left(\mathrm{CI}_{\tilde{\tau} \tilde{\rho} \tilde{\sigma} \tilde{\xi}}^{\delta \mathcal{E}}\left(\alpha_{n}, r\right), r\right) \leq \mathrm{Cl}_{\tilde{\tau} \tilde{\rho} \tilde{\sigma} \tilde{\xi}}\left(\operatorname{int}_{\tilde{\tau} \tilde{\tau} \tilde{\sigma} \tilde{\xi}}^{\odot}\left(\varepsilon_{n}, r\right), r\right)<x_{s, t, k}$. It is a contradiction for Eq. (18).

Theorem 5. Let $\left(\tilde{\mathcal{F}}, \tau \tau^{\tilde{\varrho} \tilde{\sigma} \tilde{s}}, £^{\tilde{\varrho} \tilde{\sigma} \tilde{\zeta}}\right)$ be an SVNITS, for $r \in \zeta_{0}$ and $\alpha_{n}, \varepsilon_{n} \in \zeta^{\tilde{\mathcal{F}}}$. Then the following properties hold:

(a) $\alpha_{n}$ is $\mathrm{r}-S V N P I C$ iff $\mathrm{CI}_{\tilde{\tau} \tilde{\varrho} \tilde{\sigma} \tilde{\zeta}}\left(\alpha_{n}, r\right)=\mathrm{CI}_{\tilde{\tau} \tilde{\rho} \tilde{\sigma} \tilde{\sigma}}^{\delta £}\left(\alpha_{n}, r\right)$,

(b) $\alpha_{n}$ is $\mathrm{r}-S V N S I C$ iff $\mathrm{CI}_{\tilde{\tau} \tilde{\rho} \tilde{\sigma} \tilde{\xi}}\left(\alpha_{n}, r\right)=\mathrm{CI}_{\tilde{\tau} \tilde{\tilde{\sigma}} \tilde{s} \tilde{s}}^{\delta £}\left(\alpha_{n}, r\right)$,

(c) $\alpha_{n}$ is $\mathrm{r}-S V N \alpha I O$ iff $\mathrm{CI}_{\tilde{\tau} \tilde{\rho} \tilde{\sigma} \tilde{s}}\left(\alpha_{n}, r\right)=\mathrm{CI}_{\tilde{\tau} \tilde{\rho} \tilde{\rho} \tilde{s}}^{\delta £}\left(\alpha_{n}, r\right)=\mathrm{CI}_{\tilde{\tau} \tilde{\sigma} \tilde{\sigma} \tilde{s}}^{\theta £}\left(\alpha_{n}, r\right)$.

Proof. (a) Let $\alpha_{n}$ be an $r$-SVNPIC. Then $\alpha_{n} \leq \mathrm{CI}_{\tilde{\tau} \tilde{\tilde{\rho}} \tilde{\sigma} \tilde{s}}\left(\alpha_{n}, r\right)$, and by Theorem 3 (3) and (4), we have

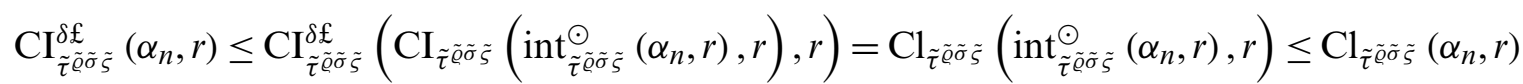

$$
\begin{aligned}
& \leq \mathrm{CI}_{\tilde{\tau} \tilde{\tilde{\sigma}} \tilde{\sigma} \tilde{s}}^{\delta £}\left(\alpha_{n}, r\right) .
\end{aligned}
$$

Conversely, suppose that there exist $v \in \tilde{\mathcal{F}}$ and $[s \in(0,1], t \in[0,1), k \in[0,1)]$ such that $\tilde{\varrho}_{\mathrm{CI}_{\tilde{\tau} \tilde{\tilde{e}}}^{\delta \varepsilon}\left(\alpha_{n}, r\right)}(v)>s>\tilde{\varrho}_{\mathrm{Cl}_{\tilde{\tau} \tilde{\varrho}}\left(\alpha_{n}, r\right)}(v), \tilde{\sigma}_{C I_{\tilde{\tau}}^{\delta \tilde{\sigma}}\left(\alpha_{n}, r\right)}(v)<t \leq \tilde{\sigma}_{C l_{\tilde{\tau} \tilde{\sigma}}\left(\alpha_{n}, r\right)}(v)$ and $\tilde{\zeta}_{C I_{\tilde{\tau} \tilde{\xi}}^{\delta \varepsilon}\left(\alpha_{n}, r\right)}(v)<k \leq$ $\tilde{\zeta}_{C l_{\tilde{\tau} \tilde{\zeta}}\left(\alpha_{n}, r\right)}(v)$. Then $x_{s, t, k}$ is not $r$ - $\delta$-cluster point of $\alpha_{n}$. So, there exists $\varepsilon_{n} \in Q_{\tau \tilde{\varrho} \tilde{\sigma} \tilde{s}}\left(x_{s, t, k}, r\right)$, with $\alpha_{n} \leq\left[\varepsilon_{n}\right]^{c}$ Since $x_{s, t, k}$ is $\mathrm{r}-\delta £$-cluster point of $\alpha_{n}$, for $\varepsilon_{n} \in Q_{\tau \tilde{\varrho} \tilde{\sigma} \tilde{s}}\left(x_{s, t, k}, r\right)$, we have int $_{\tilde{\tau} \tilde{\varrho} \tilde{\sigma} \tilde{s}}\left(\mathrm{Cl}_{\tilde{\tau} \tilde{\varrho} \tilde{\sigma} \tilde{s}}^{\odot}\left(\varepsilon_{n}, r\right), r\right) q \alpha_{n}$. Since,

$\operatorname{int}_{\tilde{\tau} \tilde{\varrho} \tilde{\sigma} \tilde{s}}\left(\mathrm{Cl}_{\tilde{\tau} \tilde{\tilde{\sigma}} \tilde{\zeta} \tilde{S}}^{\odot}\left(\varepsilon_{n}, r\right), r\right) \leq \operatorname{int}_{\tilde{\tau} \tilde{\rho} \tilde{\sigma} \tilde{s}}\left(\mathrm{Cl}_{\tilde{\tau} \tilde{\varrho} \tilde{\sigma} \tilde{s}}^{\odot}\left(\left[\alpha_{n}\right]^{c}, r\right), r\right)$,

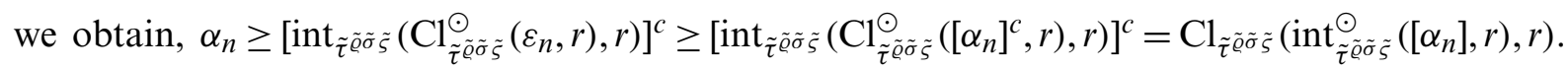

Hence, $\alpha_{n}$ is not $r-S V N I C$ set.

(b) Let $\alpha_{n}$ is an $r-S V N S I C$ set. Then, $\alpha_{n} \leq \operatorname{int}_{\tilde{\tau} \tilde{\rho} \tilde{\sigma} \tilde{s}}^{\odot}\left(\mathrm{Cl}_{\tilde{\tau} \tilde{\rho} \tilde{\sigma} \tilde{s}}\left(\left[\alpha_{n}\right]^{c}, r\right), r\right)$ and $\tau^{\tilde{\varrho}}\left(\left[\mathrm{Cl}_{\tilde{\tau} \tilde{\varrho}}\left(\left[\alpha_{n}, r\right)\right]^{c} \geq\right.\right.$

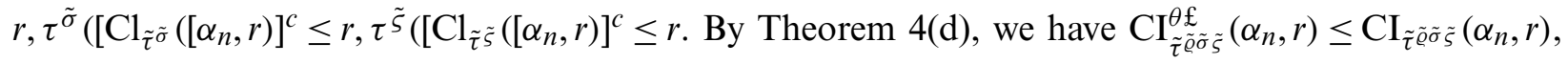

Conversely, suppose that there exist $\alpha_{n} \in \zeta^{\tilde{\mathcal{F}}}, r \in \zeta_{0}, v \in \tilde{\mathcal{F}}$ and $[s \in(0,1], t \in[0,1), k \in[0,1)]$

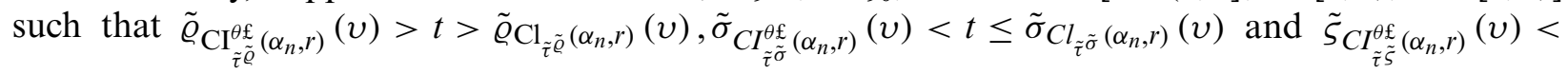
$t \leq \tilde{S}_{C l_{\tilde{\tau} \tilde{S}}\left(\alpha_{n}, r\right)}(v)$. Then $\left[\mathrm{Cl}_{\tilde{\tau} \tilde{\rho} \tilde{\sigma} \tilde{\zeta}}\left(\alpha_{n}, r\right]^{c}\right)=\operatorname{int}_{\tilde{\tau} \tilde{\varrho} \tilde{\sigma} \tilde{\zeta}}\left(\left[\alpha_{n}\right]^{c}, r\right) \in Q_{\tau \tilde{\varrho} \tilde{\sigma} \tilde{\zeta}}\left(x_{s, t, k}, r\right)$ Since $x_{s, t, k}$ is $r$ - $\theta £$-cluster point of $\alpha_{n}$, we have $\mathrm{Cl}_{\tilde{\tau} \tilde{\tilde{\sigma} \tilde{\sigma}} \tilde{\xi}}^{\odot}\left(\operatorname{int}_{\tilde{\tau} \tilde{\rho} \tilde{\sigma} \tilde{\xi}}\left(\left[\alpha_{n}\right]^{c}, r\right), r\right) q \alpha_{n}$. It implies $\alpha_{n} \not{\not}\left[\mathrm{Cl}_{\tilde{\tau} \tilde{\rho} \tilde{\sigma} \tilde{\xi}}^{\odot}\left(\operatorname{int}_{\tilde{\tau} \tilde{\rho} \tilde{\sigma} \tilde{\xi}}\left(\left[\alpha_{n}\right]^{c}, r\right), r\right)\right]^{c}=$ $\operatorname{int}_{\tilde{\tau} \tilde{\varrho} \tilde{\rho} \tilde{s}}^{\odot}\left(\mathrm{Cl}_{\tilde{\tau} \tilde{\varrho} \tilde{\sigma} \tilde{s}}\left(\alpha_{n}, r\right), r\right)$. Thus, $\alpha_{n}$ is not an $r-S V N S I C$.

(c) Similar results are shown in (a) and (b). 


\section{$4 r-\delta £-C l o s e d$ and $r-\theta £-C l o s e d$}

In this section, we firstly introduce and analyze the $r-\delta £$-closed and $r$ - $\theta £$-closed of an $\operatorname{SVNITS}\left(\tilde{\mathcal{F}}, \tau^{\tilde{\varrho} \tilde{\sigma} \tilde{\zeta}}, £^{\tilde{\varrho} \tilde{\sigma}} \tilde{\zeta}\right)$. Subsequently, we define and analyze the single-valued neutrosophic $£-$ regular and the single-valued neutrosophic almost $£$-regular of $\tilde{\mathcal{F}}$. The findings have resulted in many theorems.

Definition 16. Let $\left(\tilde{\mathcal{F}}, \tau^{\tilde{\varrho} \tilde{\sigma}} \tilde{\zeta}, £^{\tilde{\varrho} \tilde{\sigma} \tilde{\zeta}}\right)$ be an SVNITS. For $r \in \zeta_{0}$ and $\alpha_{n}, \varepsilon_{n} \in \zeta^{\tilde{\mathcal{F}}}$. Therefore,

(a) $\alpha_{n}$ is said to be $r$ - $\delta £$-closed $\left(\left[\alpha_{n}\right]_{\delta £}\right)$ [resp. $r$ - $\theta £$-closed $\left.\left[\alpha_{n}\right]_{\theta £}\right]$ iff $\mathrm{CI}_{\tilde{\tau} \tilde{\rho} \tilde{\sigma} \tilde{\xi}}^{\delta £}\left(\alpha_{n}, r\right)=\alpha_{n}$ (resp. $\left.\mathrm{CI}_{\tilde{\tau} \tilde{\tilde{\sigma}} \tilde{s} \tilde{s}}^{\theta £}\left(\alpha_{n}, r\right)=\alpha_{n}\right)$. We define

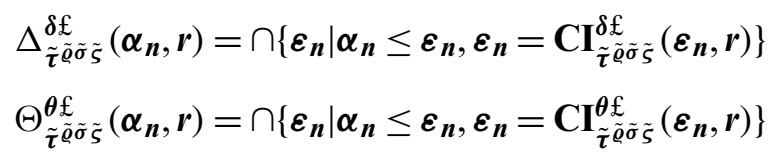

(b) The complement of $r$ - $\delta £$-closed (resp. $r$ - $\theta £$-closed) set is called $r$ - $\delta £$-open (resp. $r$ - $\theta £$-open).

Theorem 6. Let $\left(\tilde{\mathcal{F}}, \tau \tau^{\tilde{\varrho} \tilde{\sigma} \tilde{\zeta}}, £^{\tilde{\varrho} \tilde{\sigma} \tilde{\zeta}}\right)$ be an SVNITS. For $r \in \zeta_{0}$ and $\alpha_{n} \in \zeta^{\tilde{\mathcal{F}}}$. Then the following properties are holds:

(c). $\Delta_{\tilde{\tau} \tilde{\tau} \tilde{\sigma} \tilde{s}}^{\delta £}\left(\alpha_{n}, r\right)=\mathrm{CI}_{\tilde{\tau} \tilde{\rho} \tilde{\sigma} \tilde{s}}^{\delta £}\left(\alpha_{n}, r\right)$,

(d). $\Delta_{\tilde{\tau} \tilde{\rho} \tilde{\sigma} \tilde{s}}^{\delta £}\left(\alpha_{n}, r\right)$ is $\mathrm{r}-\delta £$-closed,

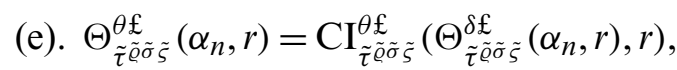

(f). $\Theta_{\tilde{\tau} \tilde{\tilde{\sigma}} \tilde{\xi} \tilde{s}}^{\theta £}\left(\alpha_{n}, r\right)$ is r- $\theta £$-closed,

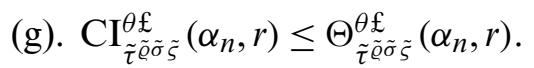

Proof. (1) Based on Theorem 4(i,j), $\alpha_{n} \leq \mathrm{CI}_{\tilde{\tau} \tilde{\varrho} \tilde{\sigma} \tilde{\zeta}}^{\delta \mathcal{E}}\left(\alpha_{n}, r\right)=\mathrm{CI}_{\tilde{\tau} \tilde{\varrho} \tilde{\sigma} \tilde{\zeta}}^{\delta £}\left(\mathrm{CI}_{\tilde{\tau} \tilde{\varrho} \tilde{\sigma} \tilde{s}}^{\delta \mathcal{E}}\left(\alpha_{n}, r\right), r\right)$, which implies

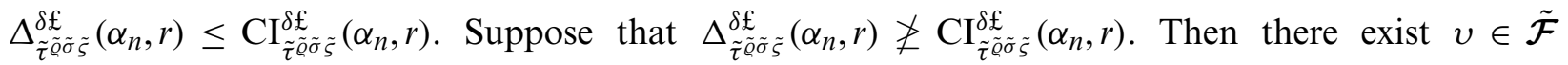
and $[s \in(0,1], t \in[0,1), k \in[0,1)]$ such that $\tilde{\varrho}_{\Delta_{\tilde{\tau} \tilde{\varrho}}^{\delta f}\left(\alpha_{n}, r\right)}(v)<s<\tilde{\varrho}_{\mathrm{CI}_{\tilde{\tau} \tilde{\varrho}}^{\delta \mathcal{E}}\left(\alpha_{n}, r\right)}(v), \tilde{\sigma}_{\Delta_{\tilde{\tau} \tilde{\sigma}}^{\delta \mathcal{f}}\left(\alpha_{n}, r\right)}(v)>t>$ $\tilde{\sigma}_{C I_{\tilde{\tau} \tilde{\tilde{\sigma}}}^{\delta \mathfrak{\tilde { \sigma }}\left(\alpha_{n}, r\right)}}(v)$ and $\tilde{\zeta}_{\Delta_{\tilde{\tau} \tilde{\zeta}}^{\delta \mathcal{E}}\left(\alpha_{n}, r\right)}(v)>k>\tilde{\zeta}_{C I_{\tilde{\tau} \tilde{\zeta}}^{\delta \mathfrak{E}}\left(\alpha_{n}, r\right)}(v)$. Based on Eq. (19), there exist $\varepsilon_{n} \in \zeta^{\tilde{\mathcal{F}}}$ and

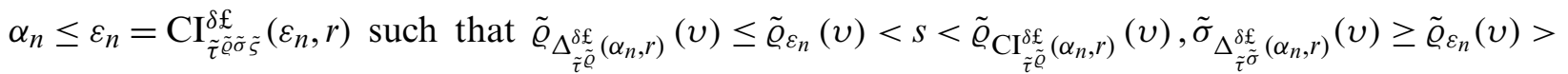
$t>\tilde{\sigma}_{C I_{\tilde{\tau} \tilde{\tilde{\sigma}}}^{\delta £}\left(\alpha_{n}, r\right)}(v)$ and $\tilde{\zeta}_{\Delta_{\tilde{\tau} \tilde{\zeta}}^{\delta \varepsilon}\left(\alpha_{n}, r\right)}(v) \geq \tilde{\varrho}_{\varepsilon_{n}}(v)>k>\tilde{\zeta}_{C I_{\tilde{\tau}}^{\delta \varepsilon}\left(\alpha_{n}, r\right)}(v)$.

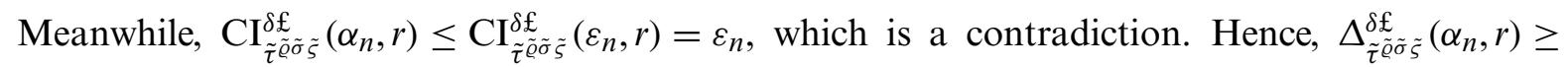
$\mathrm{CI}_{\tilde{\tau} \tilde{\varrho} \tilde{\sigma} \tilde{s}}^{\delta £}\left(\alpha_{n}, r\right)$.

(b) is similar to Theorem $4(\mathrm{k})$.

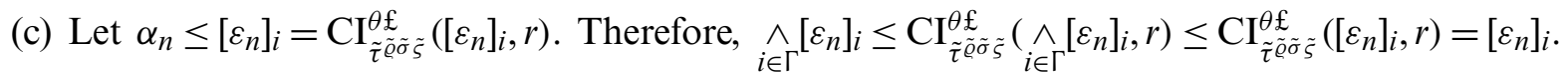

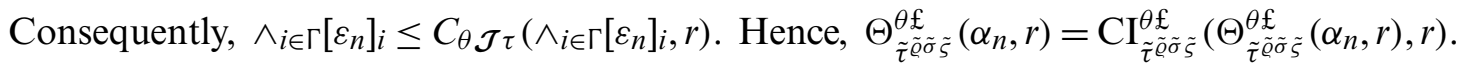

(d) It is directly obtained from (c). 


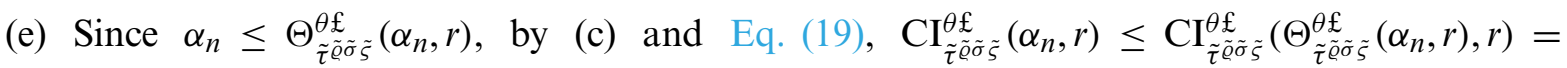

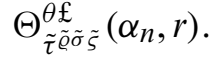

Definition 17. Let $(\tilde{\mathcal{F}}, \tau \tilde{\varrho} \tilde{\sigma} \tilde{\zeta}, £ \tilde{\varrho} \tilde{\sigma} \tilde{\zeta})$ be an SVNITS, $\alpha_{n}, \varepsilon_{n} \in \zeta^{\tilde{\mathcal{F}}}$, and $r \in \zeta_{0}$. Then $\tilde{\tilde{\mathcal{F}}}$ is called,

(a) single valued neutrosophic £-regular $\left(S V N £\right.$-regular) if for any $\alpha_{n} \in Q_{\tilde{\tau} \tilde{e} \tilde{\sigma} \tilde{\sigma}}\left(x_{s, t, k}, r\right)$, there exists $\varepsilon_{n} \in Q_{\tilde{\tau} \tilde{\varrho} \tilde{\sigma} \tilde{s}}\left(x_{s, t, k}, r\right)$ such that $\mathrm{Cl}_{\tilde{\tau} \rho \tilde{\rho} \tilde{s}}^{\odot}\left(\varepsilon_{n}, r\right) \leq \alpha_{n}$,

(b) single valued neutrosophic almost $£$-regular ( $S V N A £$-regular), if for any $\alpha_{n} \in \mathfrak{R}_{\tau \tilde{\varrho} \tilde{\sigma} \tilde{\xi}}^{£}\left(x_{s, t, k}, r\right)$, then there exists $\varepsilon_{n} \in \mathfrak{R}_{\tau \tilde{\rho} \tilde{\sigma} \tilde{\zeta}}^{\mathfrak{L}}\left(x_{s, t, k}, r\right)$ such that $\mathrm{Cl}_{\tilde{\tau} \tilde{\rho} \tilde{\sigma} \tilde{s}}^{\odot}\left(\varepsilon_{n}, r\right) \leq \alpha_{n}$.

Theorem 7. Let $\left(\tilde{\mathcal{F}}, \tau^{\tilde{\varrho} \tilde{\sigma} \tilde{\zeta}}, £^{\tilde{\varrho} \tilde{\sigma} \tilde{\zeta}}\right)$ be an SVNITS, $\alpha_{n}, \varepsilon_{n} \in \zeta^{\tilde{\mathcal{F}}}$ and $r \in \zeta_{0}$. Then the following statements are equivalent:

(a) $(\tilde{\mathcal{F}}, \tau \tilde{\varrho} \tilde{\sigma} \tilde{\varsigma}, £ \tilde{\varrho} \tilde{\sigma} \tilde{\varsigma})$ is called $S V N £$-regular,

(b) For each $x_{s, t, k} \in P_{s, t, k}(\tilde{\mathcal{F}})$ and $\alpha_{n} \in Q_{\tau \tilde{\varrho} \tilde{\sigma} \tilde{s}}\left(x_{s, t, k}, r\right)$, there exists $\varepsilon_{n} \in \mathfrak{R}_{\tau \tilde{\tilde{\sigma}} \tilde{s} \tilde{s}}^{\mathfrak{E}}\left(x_{s, t, k}, r\right)$ such that $\mathrm{Cl}_{\tilde{\tau} \tilde{\varrho} \tilde{\sigma} \tilde{\zeta}}^{\odot}\left(\varepsilon_{n}, r\right) \leq \operatorname{int}_{\tilde{\tau} \tilde{\tilde{\sigma}} \tilde{\sigma} \tilde{s}}\left(\mathrm{Cl}_{\tilde{\tau} \tilde{\rho} \tilde{\sigma} \tilde{\xi}}^{\odot}\left(\alpha_{n}, r\right), r\right)$,

(c) For each $x_{s, t, k} \in P_{s, t, k}(\tilde{\mathcal{F}})$ and each $\alpha_{n} \in Q_{\tau \tilde{\varrho} \tilde{\sigma} \tilde{s}}\left(x_{s, t, k}, r\right)$, there exists $\varepsilon_{n} \in Q_{\tau \tilde{\varrho} \tilde{\sigma} \tilde{s}}\left(x_{s, t, k}, r\right)$ such that $\mathrm{Cl}_{\tilde{\tau} \boldsymbol{\varrho} \tilde{\sigma} \tilde{\zeta}}^{\odot}\left(\varepsilon_{n}, r\right) \leq \operatorname{int}_{\tilde{\tau} \varrho \tilde{\sigma} \tilde{s}}\left(\mathrm{Cl}_{\tilde{\tau} \tilde{\varrho} \tilde{\sigma} \tilde{\zeta}}^{\odot}\left(\alpha_{n}, r\right), r\right)$,

(d) For each $x_{s, t, k} \in P_{S, t, k}(\tilde{\mathcal{F}})$ and $\mathrm{r}-S V N R I C$ set $\omega_{n} \in \zeta^{\tilde{\mathcal{F}}}$ with $x_{s, t, k} \notin \omega_{n}$, there exists $\varepsilon_{n} \in$ $Q_{\tau \tilde{\varrho} \tilde{\sigma} \tilde{s}}\left(x_{s, t, k}, r\right)$ and $\alpha_{n}$ is $\mathrm{r}-S V N \star$-open set such that $\omega_{n} \leq \alpha_{n}$ and $\mathrm{Cl}_{\tilde{\tau} \tilde{\varrho} \tilde{\sigma} \tilde{s}}^{\odot}\left(\alpha_{n}, r\right) \bar{q} \mathrm{Cl}_{\tilde{\tau}{ }^{\tilde{\rho} \tilde{\sigma} \tilde{s}}}^{\odot}\left(\varepsilon_{n}, r\right)$,

(e) For each $x_{s, t, k} \in P_{s, t, k}(\tilde{\mathcal{F}})$ and $\mathrm{r}-S V N R I C$ set $\omega_{n} \in \zeta^{\tilde{\mathcal{F}}}$ with $x_{s, t, k} \notin \omega_{n}$, there exists $\varepsilon_{n} \in$ $Q_{\tau \tilde{\varrho} \tilde{\sigma} \tilde{s}}\left(x_{s, t, k}, r\right)$ and $\alpha_{n}$ is $\mathrm{r}-S V N \star$-open set such that $\omega_{n} \leq \alpha_{n}$ and $\mathrm{Cl}_{\tilde{\tau} \tilde{\tilde{\sigma}} \tilde{\sigma} \tilde{s}}^{\odot}\left(\varepsilon_{n}, r\right) \bar{q} \alpha_{n}$,

(f) For each r-SVNRIO set $\alpha_{n} \in \zeta^{\tilde{\mathcal{F}}}$ with $\omega_{n} q \alpha_{n}$, there exists r-SVNRIO set $\varepsilon_{n} \in \zeta^{\tilde{\mathcal{F}}}$ such that $\omega_{n} q \varepsilon_{n} \leq \mathrm{Cl}_{\tilde{\tau} \tilde{\rho} \tilde{\sigma} \tilde{s}}^{\odot}\left(\varepsilon_{n}, r\right) \leq \alpha_{n}$.

(g) For each r-SVNRIC set $\alpha_{n} \in \zeta^{\tilde{\mathcal{F}}}$ with $\omega_{n} \not \leq \alpha_{n}$, there exists $\mathrm{r}-S V N R I O$ set $\varepsilon_{n} \in \zeta^{\tilde{\mathcal{F}}}$ and is $\mathrm{r}-S V N \star$-open set $\pi_{n} \in \zeta^{\tilde{\mathcal{F}}}$ such that $\omega_{n} q \varepsilon_{n}, \alpha_{n} \leq \pi_{n}$ and $\varepsilon_{n} \bar{q} \pi_{n}$.

Proof. The proof of $(\mathrm{a}) \Rightarrow(\mathrm{b})$ and $(\mathrm{b}) \Rightarrow(\mathrm{c})$ are clear.

(c) $\Rightarrow(\mathrm{a}): x_{s, t, k} \in P_{s, t, k}(\tilde{\mathcal{F}})$ and $\alpha_{n} \in \mathfrak{R}_{\tau \tilde{\tilde{\sigma}} \tilde{\tilde{s}} \tilde{f}}^{\mathfrak{f}}\left(x_{s, t, k}, r\right)$. Then, by (c), there exists $\varepsilon_{n} \in Q_{\tau \tilde{\varrho} \tilde{\sigma} \tilde{\zeta}}\left(x_{s, t, k}, r\right)$ such that $\mathrm{Cl}_{\tilde{\tau} \tilde{\rho} \tilde{\rho} \tilde{\xi}}^{\odot}\left(\varepsilon_{n}, r\right) \leq \operatorname{int}_{\tilde{\tau} \tilde{\rho} \tilde{\sigma} \tilde{\xi}}\left(\left(\mathrm{Cl}_{\tilde{\tau} \tilde{\rho} \tilde{\rho} \tilde{\sigma}}^{\odot}\left(\alpha_{n}, r\right), r\right)=\alpha_{n}\right.$. since, $\varepsilon_{n} \in Q_{\tau} \tilde{e} \tilde{\rho} \tilde{\sigma}\left(x_{s, t, k}, r\right)$ we have

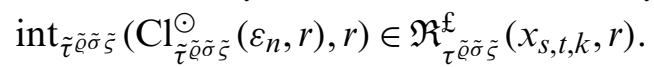

Moreover, since, $\omega_{n}=\operatorname{int}_{\tilde{\tau} \tilde{\rho} \tilde{\sigma} \tilde{\zeta}}\left(\mathrm{Cl}_{\tilde{\tau} \tilde{\varrho} \tilde{\sigma} \tilde{s}}^{\odot}\left(\varepsilon_{n}, r\right), r\right) \leq \mathrm{Cl}_{\tilde{\tau} \tilde{\tilde{\sigma}} \tilde{\bar{s}}}^{\odot}\left(\varepsilon_{n}, r\right)$, we have $\mathrm{Cl}_{\tilde{\tau} \tilde{\rho} \tilde{\sigma} \tilde{s}}^{\odot}\left(\omega_{n}, r\right) \leq \mathrm{Cl}_{\tilde{\tau} \tilde{\varrho} \tilde{\sigma} \tilde{s}}^{\odot}\left(\varepsilon_{n}, r\right)$, and hence $x_{s, t, k} q \omega_{n} \leq \mathrm{Cl}_{\tilde{\tau} \boldsymbol{\rho} \tilde{\sigma} \tilde{s}}^{\odot}\left(\omega_{n}, r\right) \leq \mathrm{Cl}_{\tilde{\tau} \tilde{\tilde{\sigma} \tilde{s}} \tilde{\rho}}^{\odot}\left(\varepsilon_{n}, r\right) \leq \alpha_{n}$ where $\omega_{n} \in \mathfrak{R}_{\tau}^{\mathcal{E} \tilde{\varrho} \tilde{\sigma} \tilde{s}}\left(x_{s, t, k}, r\right)$.

(c) $\Rightarrow(\mathrm{d})$ : Let $\omega_{n}$ be an $r-S V N R I C$ set in $\tilde{\mathcal{F}}$ and $x_{t} \in \mathrm{P}_{s, t, k}(\tilde{\mathcal{F}})$ with $x_{s, t, k} \notin \omega_{n}$. Then $x_{s, t, k} q\left[\omega_{n}\right]^{c}$ and $\left[\omega_{n}\right]^{c} \in \mathfrak{R}_{\tau \tilde{\tilde{\sigma} \tilde{\xi}} \tilde{\xi}}^{\mathfrak{L}}\left(x_{s, t, k}, r\right) \subset Q_{\tau \tilde{\varrho} \tilde{\sigma} \tilde{\xi}}\left(x_{s, t, k}, r\right)$. By (c), there exists $\pi_{n} \in Q_{\tau \tilde{\varrho} \tilde{\sigma} \tilde{s}}\left(x_{s, t, k}, r\right)$ such that $\mathrm{Cl}_{\tilde{\tau} \tilde{\varrho} \tilde{\sigma} \tilde{\zeta}}^{\odot}\left(\pi_{n}, r\right) \leq \operatorname{int}_{\tilde{\tau} \tilde{\varrho} \tilde{\sigma} \tilde{s}}\left(\mathrm{Cl}_{\tilde{\tau} \varrho \tilde{\rho} \tilde{\sigma}}^{\odot}\left(\left[\omega_{n}\right]^{c}, r\right), r\right)=\left[\omega_{n}\right]^{c}$. 
Next, $x_{s, t, k} q \operatorname{int}_{\tilde{\tau} \tilde{\rho} \tilde{\sigma} \tilde{s}}\left(\mathrm{Cl}_{\tilde{\tau} \tilde{\rho} \tilde{\sigma} \tilde{s}}^{\odot}\left(\pi_{n}, r\right), r\right)$, then $\left.\operatorname{int}_{\tilde{\tau} \tilde{\rho} \tilde{\sigma} \tilde{s}}\left(\mathrm{Cl}_{\tilde{\tau} \tilde{\rho} \tilde{\sigma} \tilde{s}}^{\odot}\left(\pi_{n}, r\right), r\right) \in Q_{\tau} \tilde{\tilde{\rho} \tilde{\sigma} \tilde{s}}\right)\left(x_{s, t, k}, r\right)$, and hence by hypothesis, there exists $\varepsilon_{n} \in Q_{\tau \tilde{\tilde{\rho} \tilde{\sigma} \tilde{\zeta}}}\left(x_{s, t, k}, r\right)$ such that $\mathrm{Cl}_{\tilde{\tau} \tilde{\tilde{\tau}} \tilde{\sigma} \tilde{\zeta}}^{\odot}\left(\varepsilon_{n}, r\right) \leq \operatorname{int}_{\tilde{\tau} \tilde{\rho} \tilde{\sigma} \tilde{\zeta}}\left(\mathrm{Cl}_{\tilde{\tau} \tilde{\rho} \tilde{\rho} \tilde{\sigma}}^{\odot}\left(\pi_{n}, r\right), r\right)$. Then, $\left.\omega_{n} \leq\left[\mathrm{Cl} 1_{\tilde{\tau} \tilde{\rho} \tilde{\sigma} \tilde{s}}^{\odot}\left(\pi_{n}, r\right), r\right)\right]^{c}$. Put $\left.\alpha_{n}=\left[\mathrm{Cl}_{\tilde{\tau} \tilde{\rho} \tilde{\sigma} \tilde{s}}^{\odot}\left(\pi_{n}, r\right), r\right)\right]^{c}$ then $\alpha_{n}$ is $r-S V N \star O$ set. Hence $\mathrm{Cl}_{\tilde{\tau} \tilde{\varrho} \tilde{\sigma} \tilde{\zeta}}^{\odot}\left(\alpha_{n}, r\right) \leq\left[\operatorname{int}_{\tilde{\tau} \tilde{\varrho} \tilde{\sigma} \tilde{s}}\left(\mathrm{Cl}_{\tilde{\tau} \tilde{\varrho} \tilde{\sigma} \tilde{\zeta}}^{\odot}\left(\pi_{n}, r\right), r\right)\right]^{c} \leq\left[\mathrm{Cl}_{\tilde{\tau} \tilde{\varrho} \tilde{\sigma} \tilde{\zeta}}^{\odot}\left(\varepsilon_{n}, r\right)\right.$.

Therefore, $\mathrm{Cl}_{\tilde{\tau} \boldsymbol{\varrho} \tilde{\sigma} \tilde{s}}^{\odot}\left(\varepsilon_{n}, r\right) \bar{q} \mathrm{Cl}_{\tilde{\tau} \boldsymbol{\varrho} \tilde{\sigma} \tilde{s}}^{\odot}\left(\alpha_{n}, r\right)$.

(d) $\Rightarrow(\mathrm{e})$ : It is trivial.

(e) $\Rightarrow$ (f): Suppose that $\alpha_{n}$ is an $r$-SVNRIO set with $\omega_{n} q \alpha_{n}$, then $\omega_{n} \not \leq\left[\alpha_{n}\right]^{c}$. Hence there exists $x_{s, t, k} \in \mathrm{P}_{s, t, k}(\tilde{\mathcal{F}})$ such that $x_{s, t, k} \in \omega_{n}$ and $\omega_{n} \not \leq\left[\alpha_{n}\right]^{c}$ where $\left[\alpha_{n}\right]^{c}$ is $r$-SVNRIC set. By (e), there exists $\varepsilon_{n} \in Q_{\tau} \tilde{e} \tilde{\sigma} \tilde{\xi}\left(x_{s, t, k}, r\right)$ and $\pi_{n} \in \zeta^{\tilde{\mathcal{F}}}$ is $r-S V N \star O$ set such that $\left[\alpha_{n}\right]^{c} \leq \pi_{n}$ and $\mathrm{Cl}_{\tilde{\tau} \tilde{\tilde{\sigma}} \tilde{\sigma} \tilde{\xi}}^{\odot}\left(\varepsilon_{n}, r\right) \bar{q} \pi_{n}$. From $\varepsilon_{n} \in Q_{\tau} \tilde{\varrho} \tilde{\sigma} \tilde{s}\left(x_{s, t, k}, r\right)$ we have $x_{s, t, k} q \varepsilon_{n} \leq \operatorname{int}_{\tilde{\tau} \tilde{\varrho} \tilde{\sigma} \tilde{s}}\left(\mathrm{Cl}_{\tilde{\tau} \rho \tilde{\rho} \tilde{s}}^{\odot}\left(\varepsilon_{n}, r\right), r\right)$.

By setting $\left[\varepsilon_{n}\right]_{1}=\operatorname{int}_{\tilde{\tau} \tilde{\rho} \tilde{\sigma} \tilde{\xi}}\left(\mathrm{Cl}_{\tilde{\tau} \tilde{\rho} \tilde{\rho} \tilde{\sigma}}^{\odot}\left(\varepsilon_{n}, r\right), r\right)$, we have $\omega_{n} q\left[\varepsilon_{n}\right]_{1}$ and $\left[\varepsilon_{n}\right]_{1}$ is $r$-SVNRIO set such that $\omega_{n} q\left[\varepsilon_{n}\right]_{1} \leq \mathrm{Cl}_{\tilde{\tau} \tilde{\rho} \tilde{\sigma} \tilde{s}}^{\odot}\left(\left[\varepsilon_{n}\right]_{1}, r\right) \leq \mathrm{Cl}_{\tilde{\tau} \tilde{\rho} \tilde{\sigma} \tilde{s}}^{\odot}\left(\varepsilon_{n}, r\right) \leq \underline{1}-\pi_{n} \leq \alpha_{n}$

(f) $\Rightarrow(\mathrm{g})$ : Let $\alpha_{n}$ be an $r$-SVNRIC set $\alpha_{n} \in \zeta^{\tilde{\mathcal{F}}}$ with $\omega_{n} \not \leq \alpha_{n}$. Therefore, $\omega_{n} q\left[\alpha_{n}\right]^{c}$ and hence by, then there exists an $r$-SVNRIO set $\varepsilon_{n} \in \zeta^{\tilde{\mathcal{F}}}$ such that $\omega_{n} q \varepsilon_{n} \leq \mathrm{Cl}_{\tilde{\tau} \tilde{\tilde{\sigma}} \tilde{\sigma} \tilde{\xi}}^{\odot}\left(\varepsilon_{n}, r\right) \leq\left[\alpha_{n}\right]^{c}$. Then, $\varepsilon_{n}$ is an $r$-SVNRIO set and $\left[\mathrm{Cl}_{\tilde{\tau} \tilde{\tilde{\sigma}} \tilde{\bar{s}} \tilde{\odot}}^{\odot}\left(\varepsilon_{n}, r\right)\right]^{c}$ is an $r-S V N \star O$ set such that $\omega_{n} q \varepsilon_{n}, \alpha_{n} \leq\left[\mathrm{Cl}_{\tilde{\tau} \tilde{\sigma} \tilde{\sigma} \tilde{s}}^{\odot}\left(\varepsilon_{n}, r\right)\right]^{c}$ and $\varepsilon_{n} \bar{q}\left[\mathrm{Cl}_{\tilde{\tau} \tilde{\varrho} \tilde{\varrho} \tilde{\zeta}}^{\odot}\left(\varepsilon_{n}, r\right)\right]^{c}$.

$(\mathrm{g}) \Rightarrow(\mathrm{a})$ : Let $\alpha_{n} \in \mathfrak{R}_{\tau \tilde{\tilde{\sigma} \tilde{\sigma}} \tilde{j}}^{\mathfrak{f}}\left(x_{s, t, k}, r\right)$ Then $x_{s, t, k} \not \leq\left[\alpha_{n}\right]^{c}$ and $\left[\alpha_{n}\right]^{c}$ is an $r$-SVNRIC set. By $(\mathrm{g})$, there exist $r$-SVNRIO set $\varepsilon_{n} \in \zeta^{\tilde{\mathcal{F}}}$ and it is $r-S V N \star O$ set $\pi_{n} \in \zeta^{\tilde{\mathcal{F}}}$ such that $x_{s, t, k} q \varepsilon_{n},\left[\alpha_{n}\right]^{c} \leq \pi_{n}$ and

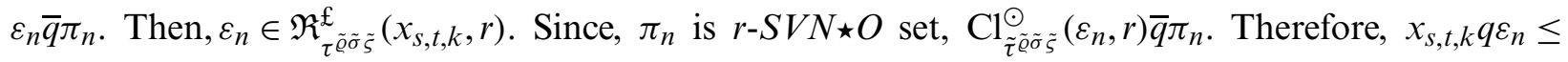
$\mathrm{Cl}_{\tilde{\tau} \tilde{\varrho} \tilde{\rho} \tilde{s}}^{\odot}\left(\varepsilon_{n}, r\right) \leq\left[\pi_{n}\right]^{c} \leq \alpha_{n}$. Hence $(\tilde{\mathcal{F}}, \tau \tilde{\varrho} \tilde{\sigma} \tilde{\zeta}, £ \tilde{\varrho} \tilde{\sigma} \tilde{\zeta})$ is $S V N £$-regular.

Theorem 8. Let $\left(\tilde{\mathcal{F}}, \tau^{\tilde{\varrho} \tilde{\sigma} \tilde{\zeta}}, £^{\tilde{\varrho} \tilde{\sigma} \tilde{\zeta}}\right)$ be an SVNITS, $\alpha_{n} \in \zeta^{\tilde{\mathcal{F}}}$ and $r \in \zeta_{0}$. Then the following statements are equivalent:

(a) $(\tilde{\mathcal{F}}, \tau \tilde{\varrho} \tilde{\sigma} \tilde{\zeta}, £ \tilde{\varrho} \tilde{\sigma} \tilde{\zeta})$ is called $S V N £$-regular,

(b) For each $x_{s, t, k} \in P_{s, t, k}(\tilde{\mathcal{F}}), \alpha_{n} \in \zeta^{\tilde{\mathcal{F}}}$ with $\tau^{\tilde{\varrho}}\left(\left[\alpha_{n}\right]^{c}\right) \geq r, \tau^{\tilde{\sigma}}\left(\left[\alpha_{n}\right]^{c}\right) \leq 1-r, \tau^{\tilde{S}}\left(\left[\alpha_{n}\right]^{c}\right) \leq 1-r$, and $x_{s, t, k} \notin \alpha_{n}$, there exists $\varepsilon_{n} \in \zeta^{\tilde{\mathcal{F}}}$ with $\varepsilon_{n}$ is $\mathrm{r}-S V N \star O$ such that $x_{s, t, k} \notin \mathrm{Cl}_{\tilde{\tau} \tilde{\varrho} \tilde{\sigma} \tilde{s}}\left(\varepsilon_{n}, r\right)$ and $\alpha_{n} \leq \varepsilon_{n}$

(c) For each $x_{s, t, k} \in P_{s, t, k}(\tilde{\mathcal{F}}), \alpha_{n} \in \zeta^{\tilde{\mathcal{F}}}$ with $\tau^{\tilde{\varrho}}\left(\left[\alpha_{n}\right]^{c}\right) \geq r, \tau^{\tilde{\sigma}}\left(\left[\alpha_{n}\right]^{c}\right) \leq 1-r, \tau^{\tilde{\zeta}}\left(\left[\alpha_{n}\right]^{c}\right) \leq 1-r$, and $x_{s, t, k} \notin \alpha_{n}$, there exists, $\varepsilon_{n} \in Q_{\tau} \tilde{e} \tilde{\sigma} \tilde{\xi}\left(x_{s, t, k}, r\right)$ and $\pi_{n} \in \zeta^{\tilde{\mathcal{F}}}$ with $\pi_{n}$ is $\mathrm{r}-S V N \star O$ such that $\alpha_{n} \leq \varepsilon_{n}$ and $\varepsilon_{n} \bar{q} \pi_{n}$

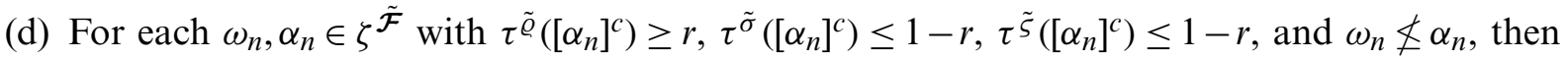
there exists $\varepsilon_{n} \in Q_{\tau} \tilde{\tilde{\sigma} \tilde{\sigma}} \tilde{\tilde{S}}\left(x_{s, t, k}, r\right)$ and $\varepsilon_{n}, \pi_{n} \in \zeta^{\tilde{\mathcal{F}}}$ with $\tau^{\tilde{\varrho}}\left(\varepsilon_{n}\right) \geq r, \tau^{\tilde{\sigma}}\left(\varepsilon_{n}\right) \leq 1-r, \tau^{\tilde{\zeta}}\left(\varepsilon_{n}\right) \leq 1-r$ and $\pi_{n}$ is $\mathrm{r}-S V N \star O$ sets such that $\omega_{n} q \varepsilon_{n}, \alpha_{n} \leq \pi_{n}$ and $\varepsilon_{n} \bar{q} \pi_{n}$. 
Proof. Similar to the proof of Theorem 7.

Theorem 9. An $\operatorname{SVNITS}\left(\tilde{\mathcal{F}}, \tau \tau^{\tilde{\varrho} \tilde{\sigma} \tilde{s}}, £^{\tilde{\varrho} \tilde{\sigma} \tilde{\zeta}}\right)$ is $S V N A £$-regular iff for each $\alpha_{n} \in \zeta^{\tilde{\mathcal{F}}}$ and $r \in$

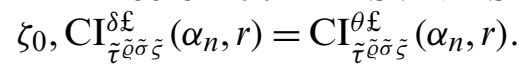

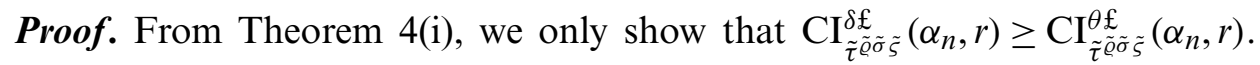

Suppose that $\mathrm{CI}_{\tilde{\tau} \tilde{\varrho} \tilde{\rho} \tilde{\xi}}^{\delta £}\left(\alpha_{n}, r\right) \nsupseteq \mathrm{CI}_{\tilde{\tau} \tilde{\varrho} \tilde{\sigma} \tilde{\xi}}^{\theta £}\left(\alpha_{n}, r\right)$. Then there exist $v \in \tilde{\mathcal{F}}$ and $[s \in(0,1], t \in[0,1), k \in$ $[0,1)]$ such that

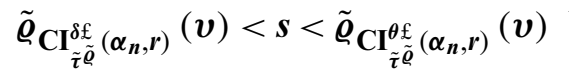

$$
\begin{aligned}
& \left.\tilde{\sigma}_{C I_{\tilde{\tau}}^{\delta \tilde{\sigma}}\left(\alpha_{n}, r\right)}(v)>\boldsymbol{t}>\tilde{\sigma}_{C I_{\tilde{\tau}}^{\theta \dot{\sigma}}\left(\alpha_{n}, r\right)}(v)\right\}
\end{aligned}
$$

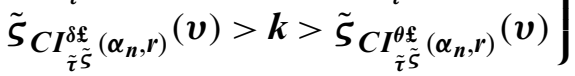

Because $\tilde{\varrho}_{\mathrm{CI}_{\tilde{\tau} \tilde{\varrho}}^{\delta \mathcal{E}}\left(\alpha_{n}, r\right)}(v)<s, \tilde{\sigma}_{\mathrm{CI}_{\tilde{\tau} \tilde{\tilde{\sigma}}}^{\delta \mathcal{E}}\left(\alpha_{n}, r\right)}(v)>t, \tilde{\zeta}_{\mathrm{C}_{\tilde{\tau} \tilde{\xi}}^{\delta \mathcal{E}}\left(\alpha_{n}, r\right)}(v)>k$, and $x_{s, t, k}$ is not an $r$ - $\delta £$-cluster point of $\alpha_{n}$. So, there exists $\varepsilon_{n} \in Q_{\tilde{\tau} \tilde{\rho} \tilde{\sigma} \tilde{s}}\left(x_{s, t, k}, r\right)$ with $\alpha_{n} \leq\left[\operatorname{int}_{\tilde{\tau} \tilde{\tau} \tilde{\sigma} \tilde{s}}\left(\mathrm{Cl}_{\tilde{\tau} \tilde{\rho} \tilde{\sigma} \tilde{s}}^{\odot}\left(\varepsilon_{n}, r\right), r\right)\right]^{c}$ Since $\varepsilon_{n} \in$

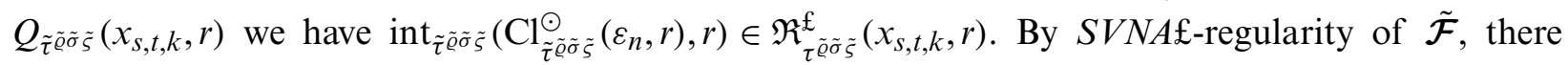
exists $\omega_{n} \in \mathfrak{R}_{\tau}^{\mathcal{E} \tilde{\varrho} \tilde{\sigma} \tilde{s}}\left(x_{s, t, k}, r\right)$ such that $\left.\mathrm{Cl}_{\tilde{\tau} \tilde{\varrho} \tilde{\sigma} \tilde{s}}^{\odot}\left(\omega_{n}, r\right), r\right) \leq \operatorname{int}_{\tilde{\tau} \tilde{\rho} \tilde{\sigma} \tilde{s}}\left(\mathrm{Cl}_{\tilde{\tau} \tilde{\rho} \tilde{\sigma} \tilde{s}}^{\odot}\left(\varepsilon_{n}, r\right), r\right)$. Thus,

$\alpha_{n} \leq\left[\operatorname{int}_{\tilde{\tau} \tilde{\rho} \tilde{\sigma} \tilde{s}}\left(\mathrm{Cl}_{\tilde{\tau} \tilde{\tau} \tilde{\sigma} \tilde{s}}^{\odot}\left(\varepsilon_{n}, r\right), r\right)\right]^{c} \leq\left[\mathrm{Cl}_{\tilde{\tau} \tilde{\rho} \tilde{\sigma} \tilde{s}}^{\odot}\left(\omega_{n}, r\right)\right]^{c}=\operatorname{int}_{\tilde{\tau} \tilde{\tau} \tilde{\sigma} \tilde{s}}^{\odot}\left(\left[\omega_{n}\right]^{c}, r\right)$,

and $\tau^{\tilde{\varrho}}\left(\omega_{n}\right) \geq r, \tau^{\tilde{\sigma}}\left(\omega_{n}\right) \leq 1-r, \tau^{\tilde{\zeta}}\left(\omega_{n}\right) \leq 1-r$. By Theorem $4(\mathrm{~d}), \tilde{\varrho}_{\mathrm{CI}_{\tilde{\tau} \tilde{\varrho}}^{\theta \mathfrak{\varrho}}\left(\alpha_{n}\right)}(v) \leq \tilde{\varrho}_{\left[\omega_{n}\right]^{c}}(v)<$ $s, \tilde{\sigma}_{C I_{\tilde{\tau}}^{\theta \notin}\left(\alpha_{n}, r\right)}(v) \geq \tilde{\sigma}_{\left[\omega_{n}\right]^{c}}(v)>t$ and $\tilde{\zeta}_{C \tilde{\tau}_{\tilde{\tau}}^{\theta \notin}\left(\alpha_{n}, r\right)}(v) \geq \tilde{\zeta}_{\left[\omega_{n}\right]^{c}}(v)>k$. It is a contradiction for Eq. (21).

Conversely, let $\alpha_{n} \in \mathfrak{R}_{\tau \tilde{\varrho} \tilde{\sigma} \tilde{\xi}}^{\mathfrak{f}}\left(x_{s, t, k}, r\right) \subset Q_{\tilde{\tau} \tilde{\varrho} \tilde{\sigma} \tilde{\xi}}\left(x_{s, t, k}\right)$. Then by Theorem $4(\mathrm{~h}), \mathrm{s}>\tilde{\varrho}_{\left[\alpha_{n}\right]^{n}}(v)=$ $\tilde{\varrho}_{\mathrm{CI}_{\tilde{\tau} \tilde{\varrho}}^{\delta \varepsilon}\left(\left[\alpha_{n}\right]^{c}, r\right)}(v), \mathrm{s}>\tilde{\varrho}_{\left[\alpha_{n}\right]^{n}}(v)=\tilde{\varrho}_{\mathrm{CI}_{\tilde{\tau}_{\tilde{\varrho}}}^{\delta \mathcal{E}}\left(\left[\alpha_{n}\right]^{c}, r\right)}(v)$ and $k<\tilde{\sigma}_{\left[\alpha_{n}\right]^{n}}(v)=\tilde{\sigma}_{C I_{\tilde{\tau}}^{\delta \varepsilon}\left(\left[\alpha_{n}\right]^{c}, r\right)}(v)$. Since,

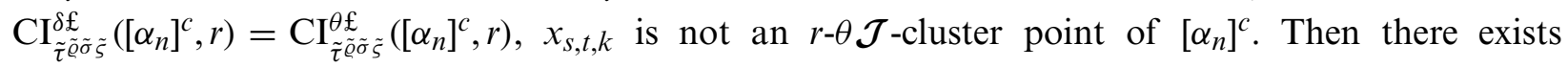
$\varepsilon_{n} \in Q_{\tilde{\tau} \tilde{\rho} \tilde{\sigma} \tilde{\xi}}\left(x_{s, t, k}, r\right)$ such that $\left[\alpha_{n}\right]^{c} \bar{q} \mathrm{Cl}_{\tilde{\tau} \tilde{\rho} \tilde{\sigma} \tilde{\xi}}^{\odot}\left(\varepsilon_{n}, r\right)$ implies $\mathrm{Cl}_{\tilde{\tau} \tilde{\rho} \tilde{\sigma} \tilde{s}}^{\odot}\left(\varepsilon_{n}, r\right) \leq \alpha_{n}=\operatorname{int}_{\tilde{\tau} \tilde{\rho} \tilde{\sigma} \tilde{\xi}}\left(\mathrm{Cl}_{\tilde{\tau} \tilde{\rho} \tilde{\sigma} \tilde{s}}^{\odot}\left(\alpha_{n}, r\right), r\right)$ and by Theorem $7(\mathrm{c}),(\tilde{\mathcal{F}}, \tau \tilde{\varrho} \tilde{\sigma} \tilde{\varsigma}, £ \tilde{\varrho} \tilde{\sigma} \tilde{\zeta})$ is $S V N A £$-regular.

Theorem 10. An $\operatorname{SVNITS}\left(\tilde{\mathcal{F}}, \tau^{\tilde{\varrho} \tilde{\sigma} \tilde{\zeta}}, £^{\tilde{\varrho} \tilde{\sigma} \tilde{\zeta}}\right)$ is $S V N A £$-regular iff for each r-SVNRIC set $\alpha_{n} \in \zeta^{\tilde{\mathcal{F}}}$

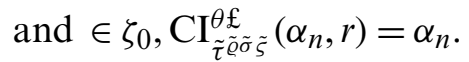

Proof. The proof is similar to Theorem 9; additionally, $r$-SVNRIC set is $r$ - $\delta £$-closed.

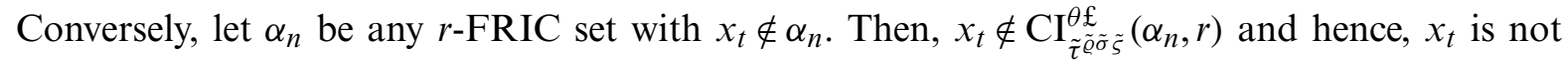
$r$ - $\theta £$-cluster point of $\alpha_{n}$ so, there there exists $\varepsilon_{n} \in Q_{\tau \tilde{\varrho} \tilde{\sigma} \tilde{\zeta}}\left(x_{s, t, k}, r\right)$ such that $\alpha_{n} \bar{q} \mathrm{Cl}_{\tilde{\tau} \tilde{\varrho} \tilde{\sigma} \tilde{\sigma}}^{\odot}\left(\varepsilon_{n}, r\right)$. Thus, $\alpha_{n} \leq\left[\mathrm{Cl}_{\tilde{\tau} \tilde{\rho} \tilde{\sigma} \tilde{s}}^{\odot}\left(\varepsilon_{n}, r\right)\right]^{c}=\omega_{n}$ and $\omega_{n}$ is $r-S V N \star O$ implies $\omega_{n} \bar{q} \mathrm{Cl}_{\tilde{\tau} \tilde{\rho} \tilde{\sigma} \tilde{s}}^{\odot}\left(\varepsilon_{n}, r\right)$. Hence, by Theorem 4(e), $(\tilde{\mathcal{F}}, \tau \tilde{\varrho} \tilde{\sigma} \tilde{\varsigma}, £ \tilde{\varrho} \tilde{\sigma} \tilde{\varsigma})$ is SVNA£-regular.

Lemma 1. If $\alpha_{n}, \varepsilon_{n} \in \zeta^{\tilde{\mathcal{F}}}, r \in \zeta_{0}$ such that $\alpha_{n} \bar{q} \varepsilon_{n}$ where $\varepsilon_{n}$ is $\mathrm{r}-\delta £$-open, then $\mathrm{CI}_{\tilde{\tau} \tilde{\tilde{\sigma}} \tilde{s} \tilde{s}}^{\delta £}\left(\alpha_{n}, r\right) \bar{q} \varepsilon_{n}$. 
Proof. Let $\alpha_{n} \bar{q} \varepsilon_{n}$ where $\varepsilon_{n}$ is $r$ - $\delta £$-open. Then, $\alpha_{n} \leq\left[\varepsilon_{n}\right]^{c}=\mathrm{CI}_{\tilde{\tau} \tilde{\varrho} \tilde{\sigma} \tilde{\xi}}^{\delta \mathcal{E}}\left(\left[\varepsilon_{n}\right]^{c}\right.$, by Theorem $4(\mathrm{k})$,

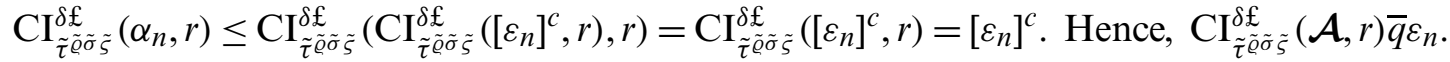

Lemma 2. Let $\left(\tilde{\mathcal{F}}, \tau^{\tilde{\varrho} \tilde{\sigma} \tilde{\zeta}}, £^{\tilde{\varrho} \tilde{\sigma} \tilde{\zeta}}\right)$ be an SVNITS and $\alpha_{n} \in \zeta^{(\tilde{\mathcal{F}}}$ is $\delta £$-open iff for each $x_{x, t, k} \in$ $Q_{\tau \tilde{\varrho} \tilde{\sigma} \tilde{\zeta}}\left(x_{s, t, k}, r\right)$ with $x_{s, t, k} q \alpha_{n}$, there exists $r$-SVNRIO set $\varepsilon_{n} \in \zeta^{\tilde{\mathcal{F}}}$ such that $x_{x, t, k} q \varepsilon_{n} \leq \alpha_{n}$.

Proof. Let $x_{x, t, k} \in \mathrm{P}_{s, t, k}\left((\tilde{\mathcal{F}}) \text { with } x_{x, t, k} q \alpha_{n} \text { Then } x_{x, t, k} \notin \alpha_{n}\right]^{c}$. Since $\alpha_{n}$ is an $r$ - $\delta £$-open set, $x_{x, t, k} \notin\left[\alpha_{n}\right]^{c}=\mathrm{CI}_{\tilde{\tau} \tilde{\varrho} \tilde{\sigma} \tilde{s}}^{\delta \mathcal{E}}\left(\left[\alpha_{n}\right]^{c}, r\right)$. Thus, $x_{x, t, k}$ is not $r$ - $\delta £$-cluster point of $\left[\alpha_{n}\right]^{c}$. So, there exists $\omega_{n} \in$

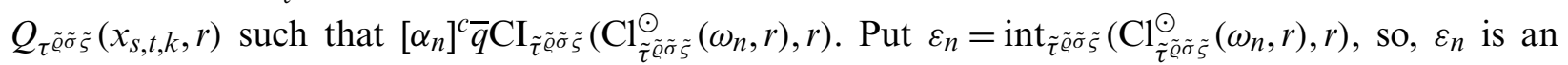
$r-S V N R I O$ set with $x_{x, t, k} q \varepsilon_{n} \leq \alpha_{n}$.

Conversely, let $\left[\alpha_{n}\right]^{c} \neq \mathrm{CI}_{\tilde{\tau} \tilde{\varrho} \tilde{\sigma} \tilde{\xi}}^{\delta \mathcal{E}}\left(\left[\alpha_{n}\right]^{c}, r\right)$, then there exist $v \in \tilde{\mathcal{F}}$ and $s, t, k \in \zeta_{0}$ such that

$$
\begin{aligned}
& \tilde{\varrho}_{\left[\alpha_{n}\right]^{c}}(v)<s<\tilde{\varrho}_{\mathbf{C I}_{\tilde{\tau}^{\delta} \tilde{\tilde{\varrho}}}^{\delta \varepsilon}\left(\left[\alpha_{n}\right]^{c}, \boldsymbol{r}\right)}(\boldsymbol{v})
\end{aligned}
$$

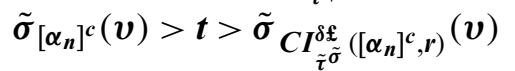

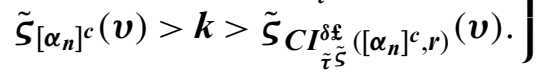

Because of $x_{x, t, k} q \alpha_{n}$, then there exists an $r-S V N R I O$ set $\varepsilon_{n}$ such that $x_{x, t, k} q \varepsilon_{n} \leq \alpha_{n}$. This implies $\left[\alpha_{n}\right]^{c} \leq\left[\varepsilon_{n}\right]^{n}=\mathrm{Cl}_{\tilde{\tau} \tilde{\rho} \tilde{\sigma} \tilde{\xi}}\left(\operatorname{int}_{\tilde{\tau} \tilde{\tilde{\rho} \tilde{\sigma} \tilde{\xi}}}^{\odot}\left(\left[\varepsilon_{n}\right]^{n}, r\right), r\right)$. By Theorem 3(d), we have $\tilde{\varrho}_{\mathrm{CI}_{\tilde{\tau}}^{\delta \varepsilon}\left(\left[\alpha_{n}\right]^{c}, r\right)}(v) \tilde{\varrho}_{\leq\left(\left[\varepsilon_{n}\right]^{n}\right)}(v)<$ $s, \tilde{\sigma}_{C I_{\tilde{\tau} \tilde{\tilde{\sigma}}}^{\delta \mathfrak{f}}\left(\left[\alpha_{n}\right]^{c}, r\right)}(v) \tilde{\sigma}_{\leq\left(\left[\varepsilon_{n}\right]^{n}\right)}(v)>t$ and $\tilde{\zeta}_{C \tilde{\tau}_{\tilde{\tau}}^{\delta \mathcal{E}}\left(\left[\alpha_{n}\right]^{c}, r\right)}(v) \tilde{\zeta}_{\leq\left(\left[\varepsilon_{n}\right]^{n}\right)}(v)>k$. It is a contradiction for Eq. (22). Hence, $\left[\alpha_{n}\right]^{c}=\mathrm{CI}_{\tilde{\tau} \tilde{\varrho} \tilde{\sigma} \tilde{s}}^{\delta £}\left(\left[\alpha_{n}\right]^{c}, r\right)$, i.e., $\alpha_{n}$ is an $r$ - $\delta £$-open set.

Lemma 3. If $\tau^{\tilde{\varrho}}\left(\alpha_{n}\right) \geq r, \tau^{\tilde{\sigma}}\left(\alpha_{n}\right) \leq 1-r, \tau^{\tilde{S}}\left(\alpha_{n}\right) \leq 1-r$, then $\mathrm{CI}_{\tilde{\tau} \tilde{\varrho} \tilde{\sigma} \tilde{s}}\left(\alpha_{n}, r\right)=\mathrm{CI}_{\tilde{\tau} \tilde{\varrho} \tilde{\sigma} \tilde{s}}^{\delta \varepsilon}\left(\alpha_{n}, r\right)$.

Proof. Follows easily by virtue of Theorem 4 .

Theorem 11. Let $\left(\tilde{\mathcal{F}}, \tau^{\tilde{\varrho} \tilde{\sigma} \tilde{\zeta}}, £^{\tilde{\varrho} \tilde{\sigma} \tilde{\zeta}}\right)$ be an SVNITS. Then the following statements are equivalent:

(a) $(\tilde{\mathcal{F}}, \tau \tilde{\varrho} \tilde{\sigma} \tilde{\zeta}, £ \tilde{\varrho} \tilde{\sigma} \tilde{\zeta})$ is $S V N A £$-regular,

(b) For each $r-\delta £$-open set $\alpha_{n} \in \zeta^{\tilde{\mathcal{F}}}$ and each $x_{x, t, k} \in \mathrm{P}_{s, t, k}(\tilde{\mathcal{F}})$ with $x_{s, t, k} q \mathcal{A}$, there exists $r-\delta £$ open set $\varepsilon_{n} \in \zeta^{\tilde{\mathcal{F}}}$ such that $x_{x, t, k} q \varepsilon_{n} \leq \mathrm{Cl}_{\tilde{\tau} \tilde{\tilde{\sigma}} \tilde{\zeta}}^{\odot}\left(\varepsilon_{n}, r\right) \leq \alpha_{n}$.

Proof. (a) $\Rightarrow\left(\right.$ b): Let $\alpha_{n}$ be $r$-fuzzy $\delta \mathcal{J}$-open set such each $x_{s, t, k} q \alpha_{n}$. Then by Lemma 3, there exists an $r$-SVNRIO set $\pi_{n} \in \zeta^{\tilde{\mathcal{F}}}$ such that $x_{s, t, k} q \pi_{n} \leq \alpha_{n}$. By $S V N A £$-regularity of $X$, there exists an $r$-FRIO set $\varepsilon_{n}$ (which is also $r$ - $\delta £$-open such that $x_{s, t, k} q \varepsilon_{n} \leq \mathrm{Cl}_{\tilde{\tau} \tilde{\varrho} \tilde{\sigma} \tilde{\sigma}}^{\odot}\left(\varepsilon_{n}, r\right) \leq \pi_{n} \leq \alpha_{n}$.

Therefore, (b) (a) is clear. 


\section{Single Valued Neutrosophic $\boldsymbol{\theta} £$-Connected}

The aim of this section is to introduce the $r$-single-valued neutrosophic $\theta £$-separated and $r$-single-valued neutrosophic $\delta £$-separated. Moreover, we introduce $r$-single-valued neutrosophic $\theta £$-connected and $r$-single valued neutrosophic $\delta £$-connected related to the $r$-single valued neutrosophic operator $\theta$ and $\delta$ defined on the set $\tilde{\mathcal{F}}$.

Definition 18. Let $\left(\tilde{\mathcal{F}}, \tau^{\tilde{\varrho} \tilde{\sigma} \tilde{\zeta}}, £^{\tilde{\varrho} \tilde{\sigma} \tilde{\zeta}}\right)$ be an SVNITS. For $r \in \zeta_{0}$ and $\alpha_{n}, \varepsilon_{n} \in \zeta^{\tilde{\mathcal{F}}}$. Then,

(a) Two non-null SVNSs $\alpha_{n}, \varepsilon_{n} \in \zeta^{\tilde{\mathcal{F}}}$ are said to be $r$-single-valued neutrosophic $\theta £$-separated if $\alpha_{n} \bar{q}\left[\varepsilon_{n}\right]_{\theta £}$ and $\varepsilon_{n} \bar{q}\left[\alpha_{n}\right]_{\theta £}$,

(b) Two non-null SVNSs $\alpha_{n}, \varepsilon_{n} \in \zeta^{\tilde{\mathcal{F}}}$ are said to be $r$-single-valued neutrosophic $\delta £$-separated if $\alpha_{n} \bar{q}\left[\varepsilon_{n}\right]_{\delta £}$ and $\varepsilon_{n} \bar{q}\left[\alpha_{n}\right]_{\delta £}$,

Remark 2. For any two non-null SVNSs $\alpha_{n}, \varepsilon_{n} \in \zeta^{\tilde{\mathcal{F}}}$, and by Eq. (8). The following implications hold: $r$-single-valued neutrosophic $\theta £$-separated $\Rightarrow r$-single-valued neutrosophic $\delta £$-separated $\Rightarrow r$ single-valued neutrosophic separated.

The following example shows that the concept of $r$-single-valued neutrosophic $\delta £$-separated is weaker than that of $r$-single-valued neutrosophic $\theta £$-separated.

Example 3. Let $\tilde{\mathcal{F}}=\{a, b, c\}$ be a set. Define $\left[\varepsilon_{n}\right]_{1},\left[\varepsilon_{n}\right]_{2} \in \zeta^{\tilde{\mathcal{F}}}$ as follows: $\left[\varepsilon_{n}\right]_{1}=\langle(1,1,0),(1,1,0),(1,1,0)\rangle ;\left[\varepsilon_{n}\right]_{2}=\langle(0,0,1),(0,0,1),(0,0,1)\rangle$.

We define an $\operatorname{SVNITS}\left(\tau^{\tilde{\varrho} \tilde{\sigma} \tilde{\zeta}}, £^{\tilde{\varrho} \tilde{\sigma} \tilde{\zeta}}\right)$ on $\tilde{\mathcal{F}}$ as follows: for each $\alpha_{n} \in \zeta^{\tilde{\mathcal{F}}}$,

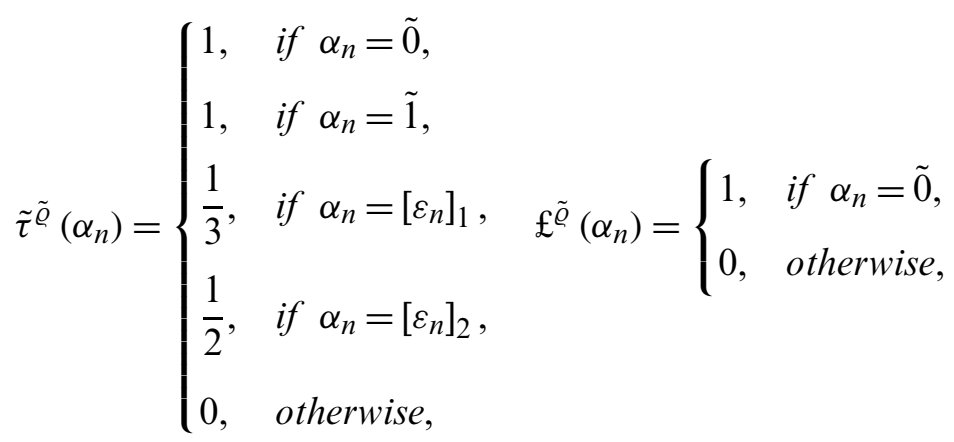

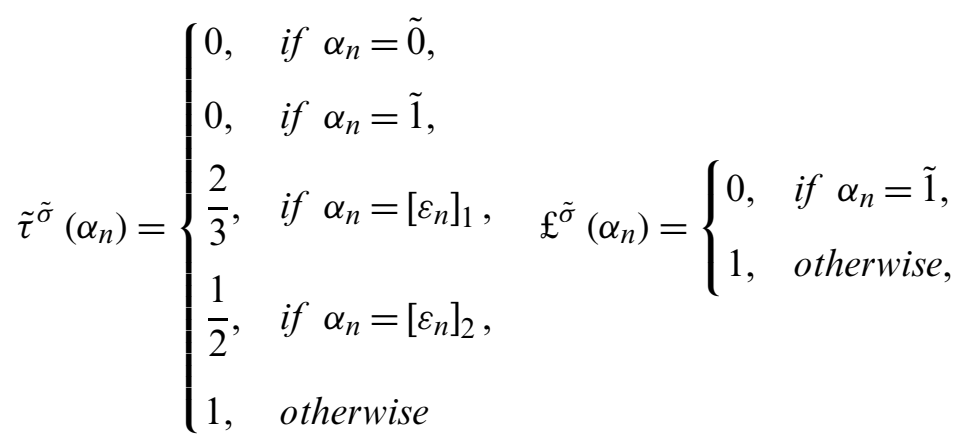




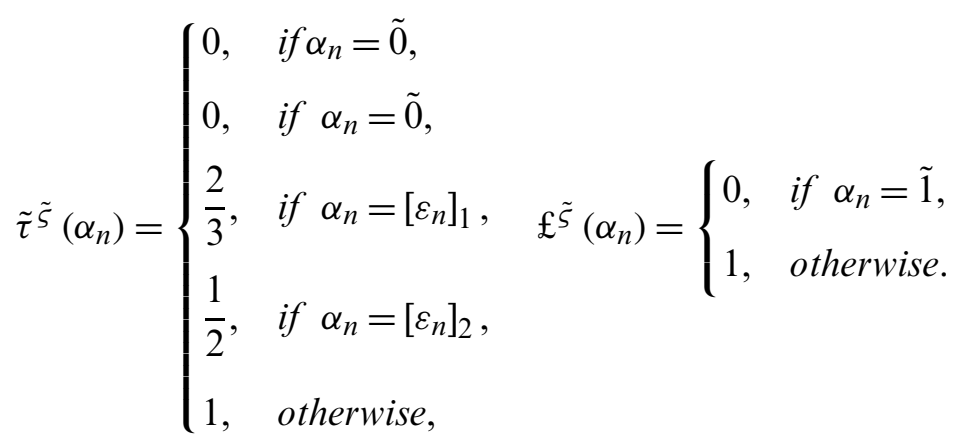

Therefore, we obtain

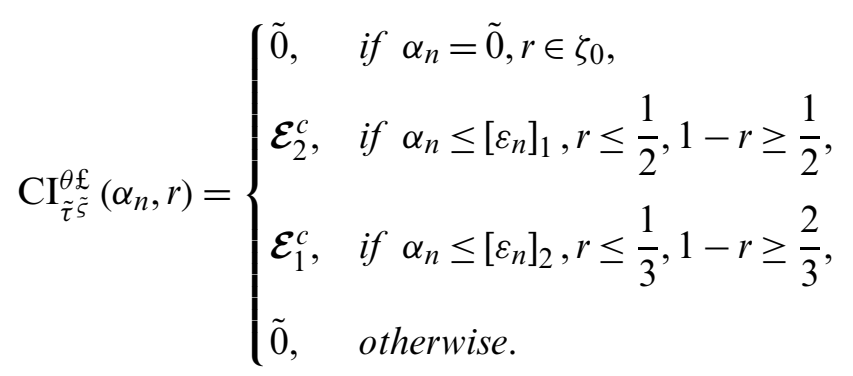

If $r \leq \frac{1}{3}$ and $1-r \geq \frac{2}{3}$, then $\left[\varepsilon_{n}\right]_{2}^{c}$ and $\left[\varepsilon_{n}\right]_{2}$ are not $\mathrm{r}$-single-valued neutrosophic $\theta £$-separated for $r \leq \frac{1}{3}$ and $1-r \geq \frac{2}{3}$. If $r>\frac{1}{3}$ and $1-r<\frac{2}{3}$, we have $\left[\varepsilon_{n}\right]_{2}^{c}$ and $\left[\varepsilon_{n}\right]_{2}$ are r-single-valued neutrosophic separated.

Theorem 12. Let $\left(\tilde{\mathcal{F}}, \tau^{\tilde{\varrho} \tilde{\sigma} \tilde{\zeta}}, £^{\tilde{\varrho} \tilde{\sigma} \tilde{\zeta}}\right)$ be an SVNITS. For $r \in \zeta_{0}$ and $\alpha_{n}, \varepsilon_{n} \in \zeta^{\tilde{\mathcal{F}}}$.

(a) If $\alpha_{n}$ and $\varepsilon_{n}$ are single-valued neutrosophic $\theta £$-separated, and $\left[\alpha_{n}\right]_{1},\left[\varepsilon_{n}\right]_{1} \in \zeta^{\tilde{\mathcal{F}}}$ such that $\left[\alpha_{n}\right]_{1} \leq \alpha_{n}\left[\varepsilon_{n}\right]_{1} \leq \varepsilon_{n}$, then $\left[\alpha_{n}\right]_{1}$ and $\left[\varepsilon_{n}\right]_{1}$ are also single-valued neutrosophic $\theta £$-separated,

(b) If $\alpha_{n} \bar{q} \varepsilon_{n}$ either both are $r-\theta £$-open or $r$ - $\delta £$-closed, then $\alpha_{n}$ and $\varepsilon_{n}$ are single-valued neutrosophic $\theta £$-separated,

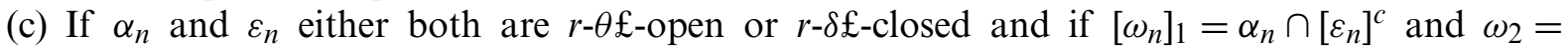
$\varepsilon_{n} \cap\left[\alpha_{n}\right]^{c}$, then $\left[\omega_{n}\right]_{1}$ and $\left[\omega_{n}\right]_{1}$ are single-valued neutrosophic $\theta £$-separated.

Proof. (a) Since $\left[\alpha_{n}\right]_{1} \leq \alpha_{n}$ we have $\left[\left[\alpha_{n}\right]_{1}\right]_{\theta £} \leq\left[\alpha_{n}\right]_{\theta £}$. Then, $\varepsilon_{n} \leq\left[\alpha_{n}\right]_{\theta £} \Rightarrow\left[\varepsilon_{n}\right]_{1} \leq\left[\alpha_{n}\right]_{\theta £} \Rightarrow$ $\left[\varepsilon_{n}\right]_{1} \leq\left[\left[\alpha_{n}\right]_{1}\right]_{\theta £}$. Similarly $\left[\alpha_{n}\right]_{1} \leq\left[\left[\varepsilon_{n}\right]_{1}\right]_{\theta £}$. Hence $\left[\alpha_{n}\right]_{1}$ and $\left[\varepsilon_{n}\right]_{1}$ are single-valued neutrosophic $\theta £$-separated.

(b) When $\alpha_{n}$ and $\varepsilon_{n}$ are $r$ - $\delta £$-closed, then $\alpha_{n}=\left[\alpha_{n}\right]_{\theta £}$ and $\varepsilon_{n}=\left[\varepsilon_{n}\right]_{\theta £}$. Since $\alpha_{n} \bar{q} \varepsilon_{n}$ we have $\left[\alpha_{n}\right]_{\theta £} \bar{q} \varepsilon_{n}$ and $\left[\varepsilon_{n}\right]_{\theta £} \bar{q} \alpha_{n}$.

When $\alpha_{n}$ and $\varepsilon_{n}$ are $r$ - $\theta £$-open, $\left[\alpha_{n}\right]^{c}$ and $\left[\varepsilon_{n}\right]^{c}$ are $r$ - $\theta £$-closed. Then $\alpha_{n} \bar{q} \varepsilon_{n} \Rightarrow \alpha_{n} \leq\left[\varepsilon_{n}\right]^{c} \Rightarrow$ $\left[\alpha_{n}\right]_{\theta £} \leq\left[\left[\varepsilon_{n}\right]^{c}\right]_{\theta £}=\left[\varepsilon_{n}\right]^{c} \Rightarrow\left[\alpha_{n}\right]_{\theta £} \bar{q} \varepsilon_{n}$. Similarly, $\left[\varepsilon_{n}\right]_{\theta £} \bar{q} \alpha_{n}$. Hence $\alpha_{n}$ and $\varepsilon_{n}$ are single-valued neutrosophic $\theta £$-separated.

(c) When $\alpha_{n}$ and $\varepsilon_{n}$ are $r-\theta £$-open, $\left[\alpha_{n}\right]^{c}$ and $\left[\varepsilon_{n}\right]^{c}$ are $r$ - $\theta £$-closed. Since $\left[\omega_{n}\right]_{1} \leq\left[\varepsilon_{n}\right]^{c},\left[\left[\omega_{n}\right]_{1}\right]_{\theta £} \leq$ $\left[\left[\varepsilon_{n}\right]^{c}\right]_{\theta £}=\left[\varepsilon_{n}\right]^{c}$ and so $\left[\left[\omega_{n}\right]_{1}\right]_{\theta £} \bar{q} \varepsilon_{n}$. Thus $\left[\omega_{n}\right]_{2} \bar{q}\left[\left[\omega_{n}\right]_{1}\right]_{\theta £}$. Similarly, $\left[\omega_{n}\right]_{1} \bar{q}\left[\left[\omega_{n}\right]_{2}\right]_{\theta £}$. Hence $\left[\omega_{n}\right]_{1}$ and $\left[\omega_{n}\right]_{1}$ are single-valued neutrosophic $\theta £$-separated. 
When $\alpha_{n}$ and $\varepsilon_{n}$ are $r$ - $\theta £$-closed, $\alpha_{n}=\left[\alpha_{n}\right]_{\theta £}$ and $\varepsilon_{n}=\left[\varepsilon_{n}\right]_{\theta £}$. Since $\left[\omega_{n}\right]_{1} \leq\left[\varepsilon_{n}\right]^{c},\left[\varepsilon_{n}\right]_{\theta £} \bar{q}\left[\omega_{n}\right]_{1}$ and hence $\left[\left[\omega_{n}\right]_{2}\right]_{\theta £} \bar{q}\left[\omega_{n}\right]_{1}$. Similarly, $\left[\left[\omega_{n}\right]_{1}\right]_{\theta £} \bar{q}\left[\omega_{n}\right]_{2}$. Hence $\left[\omega_{n}\right]_{1}$ and $\left[\omega_{n}\right]_{1}$ are single-valued neutrosophic $\theta £$-separated.

Theorem 13. Two non-null $\alpha_{n}, \varepsilon_{n} \in \zeta^{\tilde{\mathcal{F}}}$ are single-valued neutrosophic $\theta £$-separated if and only if there exist two $r$ - $\theta £$-open sets $\omega_{n}$ and $\pi_{n}$ such that $\alpha_{n} \leq \omega_{n}, \varepsilon_{n} \leq \pi_{n}, \alpha_{n} \bar{q} \pi_{n}$ and $\varepsilon_{n} \bar{q} \omega_{n}$.

Proof. Let $\alpha_{n}$ and $\varepsilon_{n}$ be single-valued neutrosophic $\theta £$-separated. Putting $\pi_{n}=\left[\left[\alpha_{n}\right]_{\theta £}\right]^{c}$ and $\omega_{n}=\left[\left[\varepsilon_{n}\right]_{\theta £}\right]^{c}$, then $\omega_{n}$ and $\pi_{n}$ are $r-\theta £$-open such that $\alpha_{n} \leq \omega_{n}, \varepsilon_{n} \leq \pi_{n}, \alpha_{n} \bar{q} \pi_{n}$ and $\varepsilon_{n} \bar{q} \omega_{n}$.

Conversely, let $\omega_{n}$ and $\pi_{n}$ be $r-\theta £$-open sets such that $\alpha_{n} \leq \omega_{n}, \varepsilon_{n} \leq \pi_{n}, \alpha_{n} \bar{q} \pi_{n}$ and $\varepsilon_{n} \bar{q} \omega_{n}$. Since $\left[\pi_{n}\right]^{c}$ and $\left[\omega_{n}\right]^{c}$ are $r$ - $\theta £$-closed, we have $\left[\alpha_{n}\right]_{\theta £} \leq\left[\pi_{n}\right]^{c} \leq\left[\varepsilon_{n}\right]^{c}$ and $\left[\varepsilon_{n}\right]_{\theta £} \leq\left[\omega_{n}\right]^{c} \leq\left[\alpha_{n}\right]^{c}$. Thus $\left[\alpha_{n}\right]_{\theta £} \bar{q} \varepsilon_{n}$ and $\left[\varepsilon_{n}\right]_{\theta £} \bar{q} \alpha_{n}$. Hence $\alpha_{n}$ and $\varepsilon_{n}$ are single-valued neutrosophic $\theta £$-separated.

Definition 19. An SVNS which cannot be expressed as the union of two single-valued neutrosophic $\theta £$-separated is said to be single-valued neutrosophic $\theta £$-connected.

Definition 20. An SVNS $\alpha_{n}$ in a SVNITS $\left(\tilde{\mathcal{F}}, \tau^{\tilde{\varrho} \tilde{\sigma} \tilde{s}}, £^{\tilde{\varrho} \tilde{\sigma} \tilde{\zeta}}\right)$ is said to be single-valued neutrosophic $\delta £$-connected if $\alpha_{n}$ cannot be expressed as the union of two single-valued neutrosophic $\delta £$-separated.

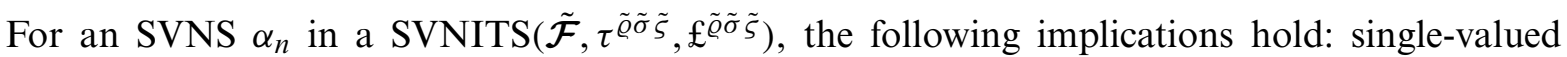
neutrosophic connected $\Rightarrow$ single-valued neutrosophic $\delta £$-connected $\Rightarrow$ single-valued neutrosophic $\theta £$-connected. If $\tau \tilde{\varrho}\left(\alpha_{n}\right) \geq r, \tau^{\tilde{\sigma}}\left(\alpha_{n}\right) \leq 1-r, \tau^{\tilde{\varsigma}}\left(\alpha_{n}\right) \leq 1-r$, then these three properties are equivalent.

Theorem 14. Let $\alpha_{n}$ be a non-null single-valued neutrosophic $\theta £$-connected in a SVNITS $\left(\tilde{\mathcal{F}}, \tau^{\tilde{\varrho} \tilde{\sigma} \tilde{\zeta}}, £^{\tilde{\varrho} \tilde{\sigma} \tilde{\zeta}}\right)$. If $\alpha_{n}$ is contained in the union of two single-valued neutrosophic $\theta £$-separated $\varepsilon_{n}$ and $\omega_{n}$, then exactly one of the following conditions (a) or (b) holds:

(a) $\alpha_{n} \leq \varepsilon_{n}$ and $\alpha_{n} \cap \omega_{n}=\tilde{0}$,

(b) $\alpha_{n} \leq \omega_{n}$ and $\alpha_{n} \cap \varepsilon_{n}=\tilde{0}$.

Proof. We first note that when $\alpha_{n} \cap \omega_{n}=\tilde{0}$, then $\alpha_{n} \leq \varepsilon_{n}$, since $\alpha_{n} \leq \varepsilon_{n} \cup \omega_{n}$. Similarly, when $\alpha_{n} \cap \varepsilon_{n}=\tilde{0}$, we have $\alpha_{n} \leq \omega_{n}$. Since $\alpha_{n} \leq \varepsilon_{n} \cup \omega_{n}$, both $\alpha_{n} \cap \varepsilon_{n}=\tilde{0}$ and $\alpha_{n} \cap \omega_{n}=\tilde{0}$ cannot hold simultaneously. Again, if $\alpha_{n} \cap \varepsilon_{n} \neq \tilde{0}$ and $\alpha_{n} \cap \omega_{n} \neq \tilde{0}$, then, by Theorem 12 (1), $\alpha_{n} \cap \omega_{n}$ and $\alpha_{n} \cap \varepsilon_{n}$ are single-valued neutrosophic $\theta £$-separated such that $\alpha_{n}=\left(\alpha_{n} \cap \varepsilon_{n}\right) \cup\left(\alpha_{n} \cap \omega_{n}\right)$, contradicting the single-valued neutrosophic $\theta £$-connectedness of $\alpha_{n}$. Hence, exactly one of the conditions (1) or (2) above must hold.

Theorem 15. Let $\left\{\left[\alpha_{n}\right]_{j} \mid j \in J\right\}$ be a collection of single-valued neutrosophic $\theta £$-connected in $\left(\tilde{\mathcal{F}}, \tau^{\tilde{\varrho} \tilde{\sigma} \tilde{s}}, £^{\tilde{\varrho} \tilde{\sigma} \tilde{\zeta}}\right)$. If there exists $i \in J$ such that $\left[\alpha_{n}\right]_{j} \cap\left[\alpha_{n}\right]_{i} \neq \tilde{0}$ for each $j \in J$, then $\alpha_{n}=\cup\left\{\left[\alpha_{n}\right]_{j} \mid j \in J\right\}$ is single-valued neutrosophic $\theta £$-connected.

Proof. Suppose that $\alpha_{n}$ is not single-valued neutrosophic $\theta £$-connected. Then there exist singlevalued neutrosophic $\theta £$-separated $\varepsilon_{n}$ and $\omega_{n}$ such that $\alpha_{n}=\varepsilon_{n} \cap \omega_{n}$. By Theorem 14, we have either (a) $\left[\alpha_{n}\right]_{j} \leq \varepsilon_{n}$ with $\left[\alpha_{n}\right]_{j} \cap \omega_{n}=\tilde{0}$ or (b) $\left[\alpha_{n}\right]_{j} \leq \omega_{n}$ with $\left[\alpha_{n}\right]_{j} \cap \varepsilon_{n}=\tilde{0}$ for each $j \in J$. Similarly, either $\left(\mathrm{a}^{\prime}\right)\left[\alpha_{n}\right]_{i} \leq \varepsilon_{n}$ with $\left[\alpha_{n}\right]_{i} \cap \omega_{n}=\tilde{0}$ or $\left(\mathrm{b}^{\prime}\right)\left[\alpha_{n}\right]_{i} \leq \omega_{n}$ with $\left[\alpha_{n}\right]_{i} \cap \varepsilon_{n}=\tilde{0}$ for each $i \in J$. We may assume, without loss of generality, that $\left[\alpha_{n}\right]_{j}$ is non-null for each $j \in J$, and hence exactly one of the conditions (a) and (b), and exactly one of $\left(a^{\prime}\right)$ and $\left(b^{\prime}\right)$ will hold.

Since $\left[\alpha_{n}\right]_{j} \cap\left[\alpha_{n}\right]_{i} \neq \tilde{0}$ for each $j \in J$, the conditions (a) and $\left(\mathrm{b}^{\prime}\right)$ cannot happen, and similarly (b) and $\left(1^{\prime}\right)$ cannot hold simultaneously. If (a) and ( $\left.\mathrm{a}^{\prime}\right)$ hold, then $\left[\alpha_{n}\right]_{j} \leq \varepsilon_{n}$ with $\left[\alpha_{n}\right]_{j} \cap \omega_{n}=\tilde{0}$. 
Then $\alpha_{n} \leq \varepsilon_{n}$ with $\alpha_{n} \cap \omega_{n}=\tilde{0}$ and thus $\omega_{n}=\tilde{0}$ a contradiction. Similarly, if (b) and ( $\left.\mathrm{b}^{\prime}\right)$ hold, then we have $\varepsilon_{n}=\tilde{0}$ again a contradiction.

Lemma 4. An $\operatorname{SVNITS}(\tilde{\mathcal{F}}, \tau \tilde{\varrho} \tilde{\sigma} \tilde{\zeta}, £ \tilde{\varrho} \tilde{\sigma} \tilde{\zeta})$ is $\operatorname{SVNA£-regular~iff~}\left[\alpha_{n}\right]_{\delta £}=\left[\alpha_{n}\right]_{\theta £}$ for every $\alpha_{n} \in \zeta^{\tilde{\mathcal{F}}}$.

Proof. Obvious.

Theorem 16. Let $\left(\tilde{\mathcal{F}}, \tau^{\tilde{\varrho} \tilde{\sigma} \tilde{s}}, £^{\tilde{\varrho} \tilde{\sigma} \tilde{\zeta}}\right)$ be an SVNITS, $\alpha_{n} \in \zeta^{\tilde{\mathcal{F}}}, r \in \zeta_{0}$. If $\left(\tilde{\mathcal{F}}, \tau^{\tilde{\varrho} \tilde{\sigma} \tilde{\zeta}}, £^{\tilde{\rho} \tilde{\sigma} \tilde{\zeta}}\right)$ is SVNA£-regular and $\alpha_{n}$ is single-valued neutrosophic $\theta £$-connected set, then $\alpha_{n}$ is single-valued neutrosophic $\delta f$-connected set.

Proof. Follows easily by virtue of Lemma 4.

Corollary 1. For a $\alpha_{n} \in \zeta^{\tilde{\mathcal{F}}}$ of SVNA£-regular space $\left(\tilde{\mathcal{F}}, \tau^{\tilde{\varrho} \tilde{\sigma} \tilde{\zeta}}, £^{\tilde{\varrho} \tilde{\sigma} \tilde{\zeta}}\right)$, the following are equivalent:

(a) $\alpha_{n}$ is $r$-single-valued neutrosophic connected,

(b) $\alpha_{n}$ is $r$-single-valued neutrosophic $\delta £$-connected,

(c) $\alpha_{n}$ is $r$-single-valued neutrosophic $\theta £$-connected.

Proof. Follows easily by virtue of Theorem 16.

\section{Conclusion}

The neutrosophic set theory has been established and applied extensively to many problems involving uncertainties. Herein, we provided clear definitions of single-valued neutrosophic operators $\mathrm{CI}_{\tilde{\tau} \boldsymbol{\varrho} \tilde{\sigma} \tilde{s}}^{\theta £}$ and $\mathrm{CI}_{\tilde{\tau} \tilde{\varrho} \tilde{\sigma} \tilde{s}}^{\delta £}$ created from an SVNI topological space $\left(\tilde{\mathcal{F}}, \tau \tilde{\varrho} \tilde{\sigma} \tilde{\zeta}, £^{\tilde{\varrho} \tilde{\sigma} \tilde{\zeta}}\right)$ and we established that $\mathrm{CI}_{\tilde{\tau} \tilde{\tilde{\sigma}} \tilde{\sigma} \tilde{\zeta}}^{\delta £}\left(\alpha_{n}, r\right)=\mathrm{CI}_{\tilde{\tau} \tilde{\rho} \tilde{\sigma} \tilde{s}}\left(\alpha_{n}, r\right)$ when $£^{\tilde{\varrho} \tilde{\sigma} \tilde{\zeta}}=£_{0}^{\tilde{\varrho} \tilde{\sigma} \tilde{s}}$. In addition, we presented the idea of $r$-singlevalued neutrosophic $\theta £$-connectedness based on a single-valued neutrosophic ideal $£^{\tilde{\varrho} \tilde{\sigma} \tilde{\zeta}}$ which has kindred with a preceding $r$-single-valued neutrosophic connectedness and the relationships among them are inspected. Moreover, we introduced an $r$-single-valued neutrosophic $\delta £$-connectedness connected to a single-valued neutrosophic $\delta$ on the set $\tilde{\mathcal{F}}$ and analyzed some of their properties. This study not only provides a hypothetical basis for additional requests in neutrosophic topology, but also for the expansion of other methodical aspects.

\section{Discussion for further works:}

The current concept can be extended by

- Investigating neutrosophic metric topological spaces;

- Investigating the products of connected and Hausdorff spaces for $\left(\tilde{\mathcal{F}}, \tau^{\tilde{\varrho} \tilde{\sigma} \tilde{\varsigma}}, £^{\tilde{\varrho} \tilde{\sigma} \tilde{\zeta}}\right)$.

Acknowledgement: The authors acknowledge Majmaah University for supporting this study. They are also grateful to the referees for their valuable comments and suggestions, which have improved the contents of this article.

Funding Statement: The authors received no specific funding for this study.

Conflicts of Interest: The authors declare that they have no conflicts of interest to report regarding the present study. 


\section{References}

1. Smarandache, F. (1999). A unifying field in logics, neutrosophy: Neutrosophic probability, set and logic. Rehoboth, NM, USA: American Research Press.

2. Bakbak, D., Uluçay, V., Şahin, M. (2019). Neutrosophic soft expert multiset and their application to multiple criteria decision making. Mathematics, 7(1), 50. DOI 10.3390/math7010050.

3. Mishra, K., Kandasamy, I., Kandasamy, V., Smarandache, F. (2020). A novel framework using neutrosophy for integrated speech and text sentiment analysis. Symmetry, 12(10), 1715. DOI 10.3390/sym12101715.

4. Wang, H., Smarandache, F., Zhang, Y. Q., Sunderraman, R. (2010). Single valued neutrosophic sets. Multispace Multistruct, 5, 410-413.

5. Kim, J., Lim, P. K., Lee, J. G., Hur, K. (2018). Single valued neutrosophic relations. Annals of Fuzzy Mathematics and Informatics, 6, 201-221.

6. Kim, J., Smarandache, F., Lee, J. G., Hur, K. (2019). Ordinary single valued neutrosophic topological spaces. Symmetry, 11(9), 1075. DOI 10.3390/sym11091075.

7. Saber, Y. M., Alsharari, F., Smarandache, F. (2020). On single-valued neutrosophic ideals in Šostak's sense. Symmetry, 12(2), 193. DOI 10.3390/sym12020193.

8. Saber, Y. M., Alsharari, F., Smarandache, F. (2020). Connectedness and stratification of single-valued neutrosophic topological spaces. Symmetry, 12(9), 1464. DOI 10.3390/sym12091464.

9. Zahran, A. M., Abd El-baki, S. A., Saber, Y. M. (2009). Decomposition of fuzzy ideal continuity via fuzzy idealization. International Journal of Fuzzy Logic and Intelligent Systems, 9(2), 83-93. DOI 10.5391/IJFIS.2009.9.2.083.

10. Das, R., Smarandache, F., Tripathy, B. (2020). Neutrosophic fuzzy matrices and some algebraic operations. Neutrosophic Sets and Systems, 32, 401-409.

11. Alsharari, F. (2021). £-Single valued extremally disconnected ideal neutrosophic topological spaces. Symmetry, 13(1), 53. DOI 10.3390/sym13010053.

12. Alsharari, F. (2020). Decomposition of single-valued neutrosophic ideal continuity via fuzzy idealization. Neutrosophic Sets and Systems, 38, 145-163.

13. Alsharari, F., Saber, Y., Smarandache, F. (2021). Compactness on single-valued neutrosophic ideal topological spaces. Neutrosophic Sets and Systems, 41, 127-145.

14. Riaz, M., Smarandache, F., Karaaslan, F., Hashmi, M., Nawaz, I. (2020). Neutrosophic soft rough topology and its applications to multi-criteria decision-making. Neutrosophic Sets and Systems, 35, 198-219.

15. Salama, A. A., Alblowi, S. A. (2012). Neutrosophic set and neutrosophic topological spaces. IOSR Journal of Mathematics, 3(4), 31-35. DOI 10.9790/5728-0343135.

16. Salama, A. A., Smarandache, F. (2015). Neutrosophic crisp set theory. USA: The Educational Publisher, Inc.

17. Salama, A. A., Smarandache, F., Kroumov, V. (2014). Neutrosophic crisp sets and neutrosophic crisp topological spaces. Neutrosophic Sets and Systems, 2, 25-30.

18. Hur, K., Lim, P. K., Lee, J. G., Kim, J. (2017). The category of neutrosophic crisp sets. Annals of Fuzzy Mathematics and Informatics, 14(1), 43-54. DOI 10.30948/afmi.

19. Hur, K., Lim, P. K., Lee, J. G., Kim, J. (2016). The category of neutrosophic sets. Neutrosophic Sets and Systems, 14, 12-20. DOI 10.5281/zenodo.570903.

20. Yang, H. L., Guo, Z. L., She, Y., Liao, X. (2016). On single valued neutrosophic relations. Journal of Intelligent \& Fuzzy Systems, 30(2), 1045-1056. DOI 10.3233/IFS-151827.

21. El-Gayyar, M. (2016). Smooth neutrosophic topological spaces. Neutrosophic Sets and Systems, 65, 65-72.

22. AL-Nafee, A. B., Broumi, S., Smarandache, F. (2021). Neutrosophic soft bitopological spaces. International Journal of Neutrosophic Science, 14(1), 47-56.

23. Muhiuddin, G. (2021). P-ideals of BCI-algebras based on neutrosophic N-structures. Journal of Intelligent \& Fuzzy Systems, 40(1), 1097-1105. DOI 10.3233/JIFS-201309.

24. Muhiuddin, G., Jun, Y. B. (2020). Further results of neutrosophic subalgebras in BCK/BCI-algebras based on neutrosophic points. Turkish World Mathematical Society Journal of Applied and Engineering Mathematics, 10(2), 232-240. 
25. Mukherjee, A., Das, R. (2020). Neutrosophic bipolar vague soft set and its application to decision making problems. Neutrosophic Sets and Systems, 32, 410-424.26.

26. Ye, J. (2014). A multicriteria decision-making method using aggregation operators for simplified neutrosophic sets. Journal of Intelligent \& Fuzzy Systems, 26, 2450-2466. 